\section{IL LASCITO DEL CARDINALE. UN RIPOSTIGLIO DI ANTONINIANI DALL'ITALIA SETENTRIONALE (?)}

\begin{abstract}
This paper concerns a nucleus of coins donated to the Republic of Venice by the Venetian Cardinal Giovan Battista Zen by 1501. Arrived at the Libreria Marciana and then unexpectedly divided between the collections of the Museo Archeologico Nazionale in Venice and the Museo Correr in Venice, this collection is now largely identifiable thanks to the archival ducumentation at the Biblioteca Marciana in Venice. The characteristics that these coins have in common make it possible a well-founded assumption that the entire nucleus donated by Zen was a hoard of denarii and antoniniani, which ends with issues of the Gallienic age. Reconstructed in this way, the hoard is comparable with many other Italian treasures, mainly from central and northern Italy, probably hoarded as a result of the continuous devaluation of the antoninianus in the ' 60 s of the third century. These comparisons allow us to locate within these areas the possible site find of the hoard, since these are also the context in which the pastoral activity of the Cardinal took place. Keywords: Gallienus - antoninianus - coin hoards - Central and Northern Italy - Museo Archeologico Nazionale di Venezia.
\end{abstract}

$\mathbf{N}$ el 1764 alla raccolta del senatore veneto Pietro Morosini (1611-1683), legata alla Serenissima nel 1683, fu unito un nucleo di monete appartenuto al Cardinale Giovan Battista Zen (1439-1501) ${ }^{1}$. Dopo il furto

${ }^{1}$ Sulla figura del Cardinale Zen si veda SORANZO 1962; JESTAZ 1995; FAVARETTO 2012. Altre notizie in ZIPPEL 1905, part. 54-55, nota 4; cfr. inoltre anche il sito web http://webdept.fiu.edu/ mirandas/bios1468. htm\#Zeno (ultimo accesso agosto 2019), con ulteriore bibliografia. Per eventuali altre informazioni e approfondimenti DEL TORRE 1997, nota 54, rinvia all'Archivio di Stato di Venezia (d'ora in poi ASV), Procuratori di San Marco, Citra, bb. 236-242. Circa le informazioni conosciute sull'unione delle sue monete antiche alla collezione numismatica di Pietro Morosini v. BERCHET 1899, 34-35 (l'autore confonde però il legato Morosini, con il dono Zen); GALLO 1950, 74; ASOLATI/

\section{Michele Asolati}

Università degli studi di Padova

michele.asolati@unipd.it

DOI: $10.14795 /$ j.v7i1_SI.475

ISSN 2360 - 266X

ISSN-L 2360 - 266X 


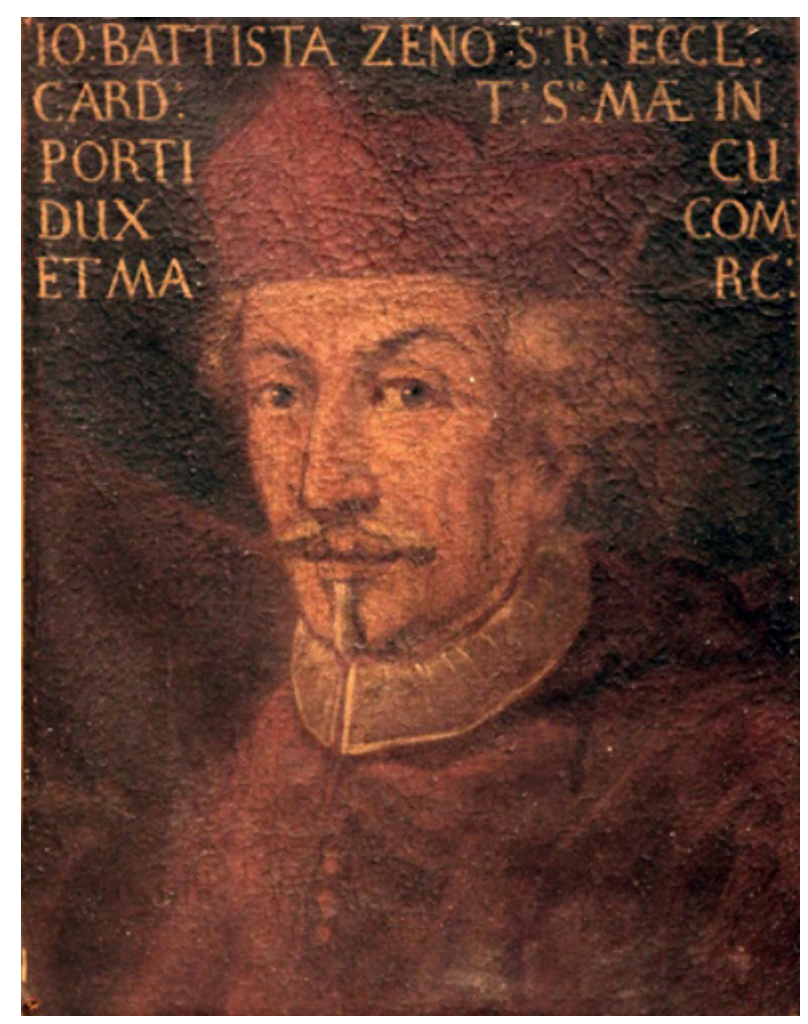

Ritratto del Cardinale Giovan Battista Zen, ricostruzione del XVII secolo (Venezia, Museo Correr, Cl. I, n. 1075)

subito nel 1687 e il riscontro sulle monete a questo scampate nel 1729, si tratta del primo intervento significativo operato sulla collezione dalle autorità della Repubblica ${ }^{2}$. Sfuggono i dettagli di questo accorpamento per via della carenza di documentazione, ma fortunatamente è sopravvissuta un'indicazione manoscritta di questo avvenimento, la quale, per quanto cursoria, inquadra il materiale con un buon grado di specificità ${ }^{3}$. Nella nota, conservata presso la Biblioteca Marciana ${ }^{4}$, si

CRISAFULLI 1996, 133, nota 29; ASOLATI 1997, 246; ASOLATI 2004, 155; GORINI 1997, 132, nota 3.

2 Sul furto e sulle vicende successive riguardanti la raccolta cfr. ASOLATI 1997, 246; ASOLATI 2004, 155156; GORINI 1997, 132-134.

3 Cfr. anche BERCHET 1899, 34-35. V. anche GALLO 1950, 74, che evidentemente riprende questa nota: "Nel 1764 il Consiglio dei X ordinava di custodire nelle Sale delle armi 332 monete antiche....che fino ad allora erano state tenute nello scrigno dei Depositi del Camerlengo alla Cassa del Consiglio dei X"; questo autore nella relativa nota 4 cita anche i Decreti 11 luglio e 18 settembre 1764 del Consiglio dei X.

4 Biblioteca Marciana di Venezia (d'ora in poi BMVe), mss. lat., Cl. XIV, n. 325 (=4737), cc. 40r-40v. Si tratta degli indici delle monete riscontrate nel 1729 dopo il furto perpetrato ai danni della raccolta numismantica ricorda infatti come fossero state aggiunte alcune centinaia di monete, specificando:

"Duecento trenta due monete d'argento lasciate all'ecc.so C. di X.ci dall'em.mo Card. Zen, e custodite alla Cassa del Cons.o med. mo da Camerlenghi, pro tempore - con dec. to dell'Ecc.so Cons.o di X.ci deman 11 Luglio 1764 demandate alla costodia del R.r alla Sale / / rilevate e riposte in cassettina a parte dall'ecc.mo Ser Antonio Savorgnan Proveditor come da dec.to $187 \mathrm{mbre} 1764$ spicca

$\begin{array}{ll}\text { Settimio Severo num.o } & : 9- \\ \text { Julia Pia } & : 5- \\ \text { Caracalla } & : 9- \\ \text { Getta } & : 3- \\ \text { Eliogabalo } & : 4- \\ \text { Julia Paula } & : 2- \\ \text { Julia Mesa } & : 5- \\ \text { Alessandro Severo } & : 13- \\ \text { Julia Mamea } & : 6- \\ \text { Massimino } & : 6- \\ \text { Gordiano Pio } & : 65- \\ \text { Filippo Padre } & : 26- \\ \text { Ottacilla } & : 2- \\ \text { Filippo Figlio } & : 16- \\ \text { Trajan Decio } & : 24- \\ \text { Erenia Etruscilla } & : 10- \\ \text { Trebonian Gallo } & : 37- \\ \text { Volusiano } & : 46- \\ \text { Valeriano } & : 18- \\ \text { Gallieno } & : 17- \\ \text { Salonina } & : 9- \\ & \mathrm{n}^{\circ} 332^{\prime \prime}\end{array}$

Questi dati permettono di recuperare i decreti del Consiglio dei $\mathrm{X}$ in virtù dei quali fu portato a compimento il trasferimento ${ }^{5}$, nei quali il nome del donatore non viene mai riportato esplicitamente, ma semplicemente ricordato attraverso la formula "Illustre

Morosini nel 1687: il manoscritto è allegato a una copia di PATIN 1683, il quale chiude con una lettera datata 1729.28.7bre, sottoscritta da Zuan.co Garzoni, Provveditor alle Sale (cc. 2r-39r.) e con l'indicazione trascritta in Appendice (cc. 40r-40v).

5 ASV, Consiglio di X, Deliberazioni, Comuni, Registri, n. 214, cc. 108r-108v (=nuova numerazione cc. 191r-191v), 11 luglio 1764; cc. 177r-177v (=nuova numerazione cc. 260r-260v), 18 settembre 1764. 
donatore". Il decreto del settembre 1764 fornisce alcuni particolari di un certo interesse ${ }^{6}$ :

"Ha prestato il Nob. Ho. Proved.r alle Sale il più adeguato adempimento al decreto di questo Cons.o ii Luglio decorso, rissultando dalla Scrittura ora letta aver egli fatto riporre nello scrigno, in cui le antiche medaglie sono custodite, le trecento e trenta due antiche monete di Argento, ch'erano depositate nello scrigno dal Nob. Ho. Camerl.o alla Cassa, e d'averle deposte secondo l'ordine del dominio degli Impera.ri in Casselle addattate, ed in Cassetta separata, onde si conservi la memoria dell'Illustre donatore, aggiungendovi pur nel libro l'inventario occorrente a regola dal Seg. io Masser alle Sale. Manifestandoci però per il pieno aggradimento del Cons.o di X.ci ad un'opera così utile ed addattata, non si può che approvare altresì l'attenzione avuta dal Nob. Ho. Proved.r alle Sale nel suo eseguire alcuni ripari nei nicchi, ove erano assicurate le armi da taglio, ed al Terrazzo nella Casa del Lido, nel che e nell'addattare le monete nello scrigno essendogli occorsa la spesa di L. $\underline{66}$ si ordina al Camerl.o alla Cassa di fargliele contare; piacendo in fine d'intendere, che il Fed.mo Marc'Antonio Guerra sostituito al Fed.mo Girolamo Salvoni supplisca con la più desiderabile attenzione alle proprie incombenze, e che le armi siano ben tenute da Capi Mastri.

E del presente sia data copia al Nob. Ho. Camerl.o alla Cassa, perché eseguisca in conformità".

\footnotetext{
6 Con il primo provvedimento si dispone la "separazione" di alcuni oggetti dalla cassa del Consiglio dei X, per alleggerire le incombenze del Camerlengo preposto: "Con quei oggetti poi precedenti che si sono intesi, riputato avendo il Mag.to pred.o alla Cassa di far passare nelle Sale di questo Cons.o le trecento trenta due piccole Medaglie dalla qualità individuata, le quali esistevano nel Scrigno intitolato de Depositi, onde dall'attenzione del Nob. Ho. Prov: alle Sale stesse fatte custodire, resti sempre nella loro esistenza la memoria dell'Illustre donatore, nel tempo stesso, che si approva piename.te la rissoluzione del Mag.to, resta animato il Prov: alle Sale pred.e di riporle, et annichiarle, come troverà conferente"; ASV, Consiglio di X, Deliberazioni, Comuni, Registri, n. 214, cc. 108r-108v (=nuova numerazione cc. 191r-191v).
}

Diviene in questo modo chiaro che, per completare questa operazione, furono appositamente realizzati degli alloggiamenti, in cui le monete furono ordinate per imperatore; fu anche steso un inventario che accompagnasse la nuova "cassella", il quale pertanto è databile con certezza al 1764 .

I particolari citati nel decreto, assieme all'elenco succinto su trascritto, permettono di riconoscere l'inventario menzionato nel decreto stesso all'interno del manoscritto che illustra il contenuto del medagliere Morisini, redatto e/o integrato nel corso dei primi decenni del XIX secolo?

Una sezione di questo registro manoscritto, infatti, del tutto autonoma rispetto alle altre e caratterizzata da una numerazione di pagina a sé stante, elenca con un notevole livello di dettaglio monete suddivise numericamente e per autorità emittente nello stesso modo riportato sopra. Per quanto non sia specificato che questa parte dell'inventario corrisponda al nucleo del Cardinale, la rispondenza pressoché esatta dei numeri e delle autorità emittenti permette non solo di istituire la coincidenza tra i due resoconti, ma anche di ipotizzare che l'elenco succinto dipenda da quello dettagliato ${ }^{8}$, il

7 [BONICELLI, G.A.], Catalogo delle medaglie d'uomini illustri Monete e Medaglie Greche e Romane, dello Scrigno D già Morosini, diviso in tre classi, BMVe, mss. it., Cl. XI, n. 330 (=7140). Le note che accompagnano questo "catalogo" nei registri della Biblioteca Marciana riportano: "NB. Questo Catalogo fu, per ordine del Bibliotecario Pietro Bettio, compilato di mano del Vicebibliotecario Gio. Antonio Bonicelli (morto circa il 1830) su tutto ciò che del Museo Morosini rimase alla Marciana, dopo il derubamento, di cui al codice precedente", ossia quello citato supra a nota 4.

8 Questa parte del manoscritto viene riprodotta integralmente nell'Appendice proposta di seguito. La lettura del documento evidenzia una incongruenza iniziale nella somma totale delle monete, poiché sono riportate due monete in più (334) rispetto al totale di 332 pezzi indicato nel resoconto breve (v. supra). Questa differenza è dovuta a un numero inferiore di esemplari ascritti a Giulia Paola (1 anziché 2 ) e a Filippo II (8 anziché 16), compensati da un numero maggiore di pezzi assegnati a Massimino I (8 anziché 6), Gordiano III (72 anziché 65) e Valeriano (20 anziché 18). Non è possibile appurare a cosa siano dovute tali discrepanze, ma nel complesso i due elenchi sono omogenei. Va 
quale sarebbe stato dunque integrato nel "catalogo" ottocentesco proprio perché già completo.

L'interesse di questa identificazione è duplice poiché da un lato diviene possibile definire le caratteristiche del lascito del Cardinale Zen, mentre dall'altro, come vedremo tra poco, se ne può individuare la presenza, per lo meno parziale, ancora all'interno delle raccolte numismatiche pubbliche veneziane.

In relazione al primo punto, di fatto le carte citate custodiscono le uniche testimonianze riguardanti il dono/lascito da parte di questo personaggio. Il cardinale Zen morì nel 1501 e la sua eredità fu al centro di una spinosa contesa tra il Papa ela Serenissima che si adoperò affinché le ultime volontà dell'alto prelato fossero eseguite in deroga ai dettami papali. L'interesse della Repubblica di Venezia era altissimo, poiché il Cardinale lasciava ingenti somme alla Dominante soprattutto per combattere le guerre conto il nemico turco; questa ricambiava la generosità permettendo che fosse eretto un monumento funebre a lui dedicato all'interno della Basilica di San Marco $^{9}$. Non è improbabile, ma al contempo non è dimostrabile, che le stesse volontà testamentarie considerassero anche il dono delle monete antiche $e^{10}$ che, dopo

sottolineato peraltro come il manoscritto riportato in Appendice sia per molti aspetti cursorio e impreciso, specie nelle descrizioni dei dritti, e come le legende dei rovesci non siano trascritte così come visibili sulle monete, ma spesso e non sempre siano completate, quando abbreviate. In pratica il testo è attendibile, ma con margini di errore difficilmente valtabili in assenza di tutti gli esemplari. Questa circostanza lascia ipotizzare anche alcuni errori di attribuzione, tanto più probabili stante la conservazione non sempre ottimale delle monete ancora presenti. Per alcune di queste, dunque, sono proposte possibili attribuzioni alternative a quelle indicate nel manoscritto stesso.

9 SORANZO 1962, 273-274. Cfr. anche AVERY 2012.

10 Il legato testamentario è la circostanza in cui è più facile far ricadere la relazione tra le monete e la Repubblica di Venezia, soprattutto perché Zen lasciò a questa stessa nella medesima occasione somme ingenti in ducati. Chiaramente non si può escludere che il Cardinale abbia donato ancora in vita il nucleo di monete antiche. A ogni modo quest'ultimo entrò in possesso delle istituzioni marciane non oltre il 1501, una lunga decantazione in circostanze che in buona ci sfuggono ${ }^{11}$, finirono per essere conservate con quelle di Pietro Morosini. Se questa eventualità è corretta, con l'esecuzione del testamento dello Zen avrebbe preso corpo la prima donazione di monete antiche a una istituzione pubblica veneziana, una delle più antiche in assoluto. Non è chiaro il motivo per cui questo atto non godette di alcuna attenzione da parte dei contemporanei e degli eruditi successivi, tanto da rimanere di fatto finora quasi del tutto sconosciuta; certamente va considerato però che se le monete nel 1764 furono custodite "pro tempore" nello scrigno dei depositi del Camerlengo alla cassa del Consiglio dei $\mathrm{X}^{12}$, plausibilmente in precedenza erano sotto il controllo di tale supremo organo criminale e di polizia della Repubblica, con aspetti di segretezza imparagonabili con altri organi dello Stato marciano.

A ognimodo, in seguito all'accorpamento del 1764 la raccolta Zen seguì il percorso della collezione Morosini che nel 1797 fu trasferita presso la Biblioteca Marciana di Venezia, dove già si trovavano altre raccolte numismatiche, dando corpo a una collezione unitaria di quanto era in possesso di istituzioni pubbliche veneziane $^{13}$. Nel corso dell'Ottocento questa accolse anche le monete di Domenico Ascanio Molin, che nel 1886 furono separate in modo

anno della morte di Giovan Battista Zen. Molto rimane da comprendere circa l'eredità testamentaria di Zen in quanto manca a oggi un'analisi puntuale ed esaustiva delle varie versioni del testamento e dei numerosi codicilli, nonché delle varie copie sopravvissute; il testamento originale si conserva presso l'ASV, Notarile, Testamenti, P. Bon, b.131, 78 e fol. 10-15, in copia del XVIII secolo alla BMVe, cl. VII, n. 480 (=7785), c. 185 oppure presso la Biblioteca del Museo Correr di Venezia, Ms. P.D.c 916, Testamenti, T. I, fascicoli 26-28.

11 Come detto, le monete della raccolta Zen prima del loro trasferimento erano conservate presso i depositi del Camerlengo alla cassa del Consiglio dei X, ma non è dato sapere per quanto siano rimaste in quelle condizioni o se fossero collocate altrove in precedenza: da testo del decreto dell'11 luglio 1764 (v. supra, nota 6) parrebbe intendersi che fossero rimaste sempre nella detta cassa, ma l'espressione "pro tempore", utilizzata nell'elenco cursorio, insinua qualche dubbio in merito.

12 GALLO 1950, 74.

13 GORINI 1997, 134-135; ASOLATI 1997, 246; ASOLATI 2004, 156. 
non filologico per essere destinate al Comune di Venezia, ossia al Museo Correr ${ }^{14}$.

Quanto sopravvisse alla Libreria Marciana entrò a far parte del Museo Archeologico Nazionale di Venezia, formatosi nei primi decenni del Novecento ${ }^{15}$, dove ancora oggi si trova ${ }^{16}$. La complicata vicenda che caratterizzò il passaggio della raccolta Molin al Museo Correr non fu priva di conseguenze e, come noto, portò al mescolamento di parte della raccolta Molin con le altre già presenti alla Biblioteca Marciana, di fatto convogliando al Museo Correr monete che non erano della Molin e lasciando alla Libreria Marciana monete che lo erano. Questi accadimenti, dimostrati in numerose circostanze $^{17}$, sono ora documentabili anche nel caso della collezione del Cardinale Zen, proprio grazie all'elenco dettagliato su citato e alle caratteristiche fisiche delle monete a questo oggi riconducibili.

Tra le monete attualmente presenti presso le collezioni numismatiche del Museo Archeologico Nazionale di Venezia, riferibili al periodo circoscritto nei documenti citati (fine II-secondo trentennio del III secolo d.C.), si possono identificare, per la caratterizzazione della patina e della conservazione, numerosi esemplari $^{18}$ che già nel 1971 Richard Reece

\footnotetext{
$14 \mathrm{Su}$ questo aspetto e sulle conseguenze dello spostamento della raccolta numismatica di Domenico Ascanio Molin dalla Libreria Marciana al Museo Correr cfr. GAMBIER 1986; GORINI 1997; CRISAFULLI 2006; ASOLATI 2004.

15 RAVAGNAN 1997, 71-73.

16 Fatta eccezione per le monete di epoca medievale e moderna che nel 1975 furono trasferite presso la Galleria Giorgio Franchetti alla Ca' d'Oro: GORINI 1997, 134-135; ASOLATI 2004, 157.

17 Si veda per esempio CRISAFULLI 2006; ASOLATI 2009.

18 Si tratta di prodotti di corrosione di colore verde dovuto all'ampia presenza di rame nella lega; tale corrosione ingloba in moltissimi casi anche residui di terra e talvolta l'insieme di questi elementi appesantisce la superficie della moneta a tal punto da renderne difficile la lettura. Va specificato che, stabilito il carattere dominante della patina, l'individuazione dei pezzi è stata effettuata senza una verifica preliminare nel manoscritto; quest'ultima è stata condotta solo in seguito e ha dato come esito la corrispondenza di ciascun esemplare individuato a uno di quelli descritti,
}

aveva suggerito appartenere a due distinti gruzzoli rispettivamente di denari e di antoniniani ${ }^{19}$ e che più di recente lo scrivente e Cristina Crisafulli avevano riferito a un solo nucleo ${ }^{20}$. In quelle occasioni non si era considerata l'eventualità che monete con le stesse caratteristiche potessero essere presenti anche presso le raccolte numismatiche del vicino Museo Correr di Venezia. In realtà la verifica condotta proprio per questo studio ha portato all'individuazione di un buon numero di pezzi confluiti nella raccolta civica e con questa integrati ${ }^{21}$ : la riconoscibilità rimane comunque legata alla patina comune a tutti questi esemplari e alla loro rispondenza agli elenchi citati.

Va precisato che delle oltre 330 monete della collezione Zen, ne sono state riconosciute all'interno dei medaglieri dei due musei poco meno di 200. Considerata la patina dei pezzi identificati, va precisato che non sono riconoscibili altri esemplari con minori margini di sicureza. Ne deriva che poco più di 130 monte sono andate disperse, plausibilmente tra il 1764, data dell'accorpamento con la raccolta Morosini, e il secondo dopoguerra. In particolare nel corso dell'Ottocento è noto che le collezioni museali ancorché pubbliche scambiassero con altre istituzioni o con privati monete ritenute "duplicati"22; d'altro canto, i due conflitti mondiali hanno comportato spostamenti

confermando la correttezza dell'individuazione stessa. Rispetto agli esemplari individuati in ASOLATI/ CRISAFULLI 1996, 138-139 (Tab. n. 1), sono stati però esclusi gli esemplari del Museo Archeologico Nazionale di Venezia inv. nn. 971 e 992 ascrivibili a Caracalla, la cui patina, più scura di quella delle altre monete, ma anch'essa connotata per evidenti corrosioni di colore verde, aveva indotto allora anche chi scrive a considerare i pezzi come parte del nucleo, pur suscitando qualche dubbio. L'assenza dall'elenco manoscritto di questi tipi, rispettivamente RIC 108 e 26a, permette ora di consolidare quel sospetto e di espungere i due pezzi.

19 REECE 1971, 176. Cfr. anche GORINI 1992.

20 ASOLATI/CRISAFULLI 1996, 132-133, 138-139

(Tab. n. 1), 141.

${ }^{21}$ Una prima notizia di questa individuazione è data in CRISAFULLI 2006, 142, nota 12.

22 In merito a questi aspetti cfr. GORINI 1987, 245; CRISAFULLI 2006, 137; ASOLATI 2011, 521-522. 
improvvisi e non sufficientemente pianificiati delle collezioni venete, determinando probabilmente situazioni non più recuperabili.

Nondimeno, malgrado queste mancanze documentarie, le descrizioni proposte nel manoscritto sono in moltissimi casi sufficientemente precise da permettere una classificazione anche di gran parte dei pezzi oggi irrintracciabili. In questo modo si può giungere a ricomporre pressoché nella sua interezza il dono di Giovan Battista Zen, nonostante i secoli di oblio e le inopinate frammentazione e integrazione nelle raccolte dei due Musei.

Al di là delle implicazioni relative alla storia del collezionismo numismatico, che non interessano in questa sede, l'elemento forse di maggior interesse che si coglie nell'esaminare queste monete riguarda proprio le peculiarità compositive e cronologiche, da leggere inevitabilmente alla luce delle caratteristiche esteriori che connotano tutti gli esemplari. Le monete più antiche sono generalmente più consumate di quelle più recenti; vi sono varie tipologie ripetute per molte delle autorità emittenti registrate; si tratta soltanto di nominali argentei; la cronologia è relativamente stretta e le autorità emittenti sono tutte consecutive. È piuttosto evidente, dunque, che non fu operata una selezione sulla base di connotazioni esteriori (patine nobili, conservazione eccellente), o del pregio intrinseco dei pezzi, o ancora della rarità. Tutti questi elementi, unitamente alla patina omogenea, portano a concludere che l'intera collezione sia il frutto del rinvenimento di un gruzzolo, con ogni probabilità interamente donato alla Serenissima.

Per quanto sia conosciuta la sua inclinazione per le lettere ${ }^{23}$ e l'arte $^{24}$, non sono noti interessi antiquari di Giovan Battista

\footnotetext{
${ }^{23}$ ZIPPEL 1904, 55, nota 4.

24 FAVARETTO 2012, 61, 63-70. In riferimento alla collezione di arazzi di Giovan Battista Zen cfr. DOLCINI 1999. JESTAZ 1995, 333 ritiene che queste sue inclinazioni non fossero dettate da interessi reali in questi ambiti, ma piuttosto che "fossero l'espressione... del carattere fastoso di un principe della Chiesa molto attaccato alla sua dignità".
}

Zen, anche se è immaginabile che abbia avuto una formazione in tal senso, stante la stretta relazione con lo zio Pietro Barbo, divenuto papa con il nome di Paolo $\mathrm{II}^{25}$. È dunque legittimo pensare che la presenza di questo nucleo di monete antiche nel patrimonio del Cardinale fosse frutto di circostanze occasionali, ossia fosse dovuto a un rinvenimento presso una delle proprietà di famiglia o presso una delle sedi dove esercitò le proprie funzioni, o presso le quali poteva vantare diritti di commenda e prebenda ${ }^{26}$. Chiaramente le possibilità sono molteplici, ma in estrema sintesi si può "limitare" l'attenzione sostanzialmente al Veneto, alla Romagna e ad alcune aree del Lazio, compresa la stessa Roma, dove si fece erigere un palazzo attiguo alla Basilica di San Pietro $^{27}$.

Dando per acquisito, come ipotesi di lavoro, che il gruzzolo sia completo nella configurazione delineata dal manoscritto, se ne possono definire caratteristiche e cronologia sulla base di quanto su esposto e della classificazione del materiale oggi reperibile e di ciò che è possibile riconoscere dalle descrizioni manoscritte. Si tratta di un gruzzolo di denari e antoniniani databili tra l'ultimo decennio del II secolo e la seconda metà degli anni '60 del III secolo (Tabella 1). I denari coprono la prima fase da Settimo Severo a Massimino I, mentre l'antoniniano domina dal 244 in poi, anche se si può documentare almeno un denario di Gordiano III (Cartella B, n. 63). Circa la data di chiusura, le monete certamente riconoscibili di Gallieno e di Salonina non si spingono oltre il 267 d.C., anno in cui si colloca l'VIII emissione mediolanense di Gallieno, documentata certamente con un solo esemplare (Cartella D, n. 111). Assumiamo dunque come datazione più probabile per il termine del gruzzolo il 267 d.C.; stante l'assenza di coniazioni successive, assai più comuni in Italia di quelle

\footnotetext{
25 FAVARETTO 2012, 60. Sulla figura di Paolo II e le sue raccolte di antichità v. FAVARETTO 1990, pp. 4955 con bibliografia precedente.

26 SORANZO 1962; JESTAZ 1995; FAVARETTO 2012, 63.

27 SORANZO 1962, 257; JESTAZ 1995, 335.
} 
Tabella 1. Quadro distributivo delle monete appartenenti al ripostiglio del Cardinale Zen

\begin{tabular}{|c|c|c|c|c|c|c|c|c|c|c|}
\hline & \multirow{2}{*}{$\begin{array}{l}\text { Tre } \\
A n \\
\end{array}$} & \multicolumn{2}{|c|}{$\mathrm{Rm}$} & \multirow{2}{*}{$\frac{\text { Med }}{A n}$} & \multirow{2}{*}{$\frac{\mathrm{Vm}}{A n}$} & \multirow{2}{*}{$\frac{C y z}{A n}$} & \multirow{2}{*}{$\frac{\text { Ant }}{A n}$} & \multirow{2}{*}{$\frac{\text { Eme }}{D}$} & \multirow{2}{*}{$\begin{array}{l}\text { n.i. } \\
A n\end{array}$} & \multirow{2}{*}{ TOT. } \\
\hline & & $D$ & $A n$ & & & & & & & \\
\hline Settimio Severo & & 8 & & & & & & 1 & & 9 \\
\hline Giulia Domna & & 5 & & & & & & & & 5 \\
\hline Caracalla & & 9 & & & & & & & & 9 \\
\hline Geta & & 3 & & & & & & & & 3 \\
\hline Elagabalo & & 4 & & & & & & & & 4 \\
\hline Giulia Paola & & 1 & & & & & & & & 1 \\
\hline Giulia Mesa & & 5 & & & & & & & & 5 \\
\hline Severo Alessandro & & 13 & & & & & & & & 13 \\
\hline Giulia Mamea & & 6 & & & & & & & & 6 \\
\hline Massimino I & & 8 & & & & & & & & 8 \\
\hline Gordiano III & & 2 & 66 & & & & 3 & & 1 & 72 \\
\hline Filippo I & & & 26 & & & & & & & 26 \\
\hline Filippo II & & & 8 & & & & & & & 8 \\
\hline Otacilia Severa & & & 2 & & & & & & & 2 \\
\hline Traiano Decio & & & 24 & & & & & & & 24 \\
\hline Erannia Etruscilla & & & 10 & & & & & & & 10 \\
\hline Treboniano Gallo & & & 32 & & & & 2 & & 3 & 37 \\
\hline Volusiano & & & 46 & & & & & & & 46 \\
\hline Valeriano & & & 4 & & 8 & & 1 & & 7 & 20 \\
\hline Gallieno & & & 3 & 2 & 1 & & & & 11 & 17 \\
\hline Salonina & 1 & & 2 & 2 & & 1 & & & 3 & 9 \\
\hline TOT. & 1 & & & 4 & 9 & 1 & 6 & 1 & 25 & 334 \\
\hline
\end{tabular}

delle fasi gallieniche precedenti ${ }^{28}$, crediamo si possa ritenere questa data quale quella dell'interramento.

Il gruzzolo è fortemente sproporzionato a vantaggio delle emissioni più antiche fino a Gordiano III che da solo conta oltre 70 esemplari, ossia più del $20 \%$ dell'intero gruzzolo. Le emissioni di Valeriano e di Gallieno sono comunque numerose e con 47 pezzi ammontano a circa il $14 \%$. Tale struttura corrisponde a un modello di tesaurizzazione documentato da vari ripostigli italiani di età gallienica, localizzati in buona parte del territorio della penisola e nelle isole, ossia Cerretto Lomellina (PV) ${ }^{29}$, S. Michele in Lodivecchio (LO) $)^{30}$, Falerone ${ }_{28}$ CRISAFULLI 2016, 73.

29 BERNABEI 1988; VISMARA 1994, nota 74; MARTINI 1995.

30 MARTANI 1892; PENSA 1985.
$(\mathrm{FM})^{31}$, Testaccio ${ }^{32}$, Pombia (NO) $)^{33}$, Ostia $\mathrm{III}^{34}$, Portotorres $(\mathrm{SS})^{35}$, i quali tutti si distinguono appunto per la predominanza di emissioni più antiche rispetto a quelle con cui chiudono ${ }^{36}$.

Peraltro, anche a San Michele in Lodivecchio c'è una buona quantità di denari, ma il ripostiglio del Cardinale si distingue da tutti gli altri per la presenza di 64 denari pari al $19 \%$ del tesoretto. È piuttosto evidente che queste monete in particolare fossero tesaurizzate per il loro contenuto intrinseco, notevolmente più elevato di quello degli

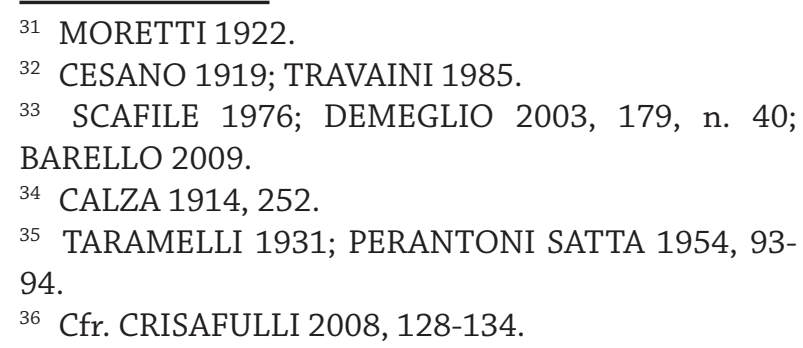


Tabella 2. Ripostigli di età gallienica rinvenuti in Italia; sono cosiderati i gruzzoli di cui si conosce il contenuto con un buon livello di dettaglio; sono elencati soltanto quelli con più di 100 esemplari (dati desunti da CRISAFULLI 2008, Catalogo dei ripostigli).

\begin{tabular}{|c|c|c|c|c|c|c|c|c|c|c|c|c|c|c|}
\hline & Gal I & Tre & $\mathrm{Rm}$ & $\mathrm{Rm} 2$ & Med & Vim & Sis & Cyz & Lao & Ant & Eme & OR II & n.d. & TOT \\
\hline Pombia (NO) & 12 & & 216 & & 56 & 3 & & & & 13 & & 1 & 16 & 317 \\
\hline Gattinara (VC) & 5 & & 54 & 1 & 163 & 2 & & & & 3 & & 2 & & 230 \\
\hline Alto vercellese & 8 & & 39 & 1 & 109 & 3 & & & & 2 & & & & 162 \\
\hline Torino & & 7 & 539 & 3 & 1195 & 7 & 3 & & & 8 & & 8 & 395 & 2165 \\
\hline $\begin{array}{l}\text { S. Michele in } \\
\text { Lodivecchio (LO) }\end{array}$ & 8 & & 715 & & 19 & 2 & & & 1 & 32 & & & 13 & 789 \\
\hline Pavia 1868 & & & 503 & & 186 & & 5 & & & 1 & & & 3 & 698 \\
\hline Ceretto Lomellina (PV) & 17 & & 246 & & 18 & 8 & & & & 8 & & 2 & & 299 \\
\hline Ottobiano (PV) & 6 & & 44 & & 380 & & & & & 3 & & 2 & 4 & 441 \\
\hline Falerone (FM) & 55 & & 4559 & 58 & 822 & 75 & & & & 285 & & 533 & 1204 & 7591 \\
\hline Testaccio & & & 735 & & 4 & & & & & 9 & & 37 & 19 & 804 \\
\hline Ostia III & & & 50 & & 4 & 1 & & & & 27 & & 9 & 222 & 313 \\
\hline Rip. Cardinale & & 1 & 287 & & 4 & 9 & & 1 & & 6 & 1 & & 25 & 334 \\
\hline
\end{tabular}

antoniniani successivi al 253 d.C.

Nonostante l'Italia, e in particolare quella nord-orientale, fosse particolarmente esposta alle incursioni barbariche e militari dopo la metà del III secolo, i caratteri del nostro come degli altri ripostigli citati induce a considerare l'eventualità che vi fossero ragioni economiche alla base del loro accumulo ${ }^{37}$, piuttosto che cause strettamente militari e contingenti che invece sembrerebbero doversi presupporre per un altro gruppo di gruzzoli di età gallienica, localizabili in ambito padano, $\mathrm{i}$ quali però contengono essenzialmente monete di Gallieno stesso, con una prevalenza di pezzi di qualità scadente ${ }^{38}$. Il 266 d.C., infatti,

37 In tal senso cfr. CRISAFULLI 2008, part. 134.

$38 \mathrm{Si}$ vedano $\mathrm{i}$ ripostigli di Ottobiano (PV) (CHIARAVALLE 1986; CHIARAVALLE 1989), Alto vercellese (FERRETTI 1986; DEMEGLIO 2003, 177, n. 22), Gattinara (VC) (FERRETTI 1994; DEMEGLIO 2003, 178-179, n. 35), Mortara (PV) (GARIBOLDI 2001), Pavia 1868 (VISMARA 2002), Torino (ASSANDRIA 1915, 62-64; CORNAGGIA 1918; DEMEGLIO 2003, 180, n. 47), Buzet, Istria (FMRHr, XVIII, 20/2), il cui interramento è forse da mettere in relazione con gli eventi bellici legati alla ribellione di Aureolo a Milano, compatibile cronologicamente e geograficamente. Sullo svolgimento di tale evento e sula sua cronologia la letteratura non è unanime; per alcuni studiosi si sarebbe sviluppato per pochi mesi nel corso del 268 (DRINKWATER 2005, 47), mentre per segna uno spartiacque nella produzione dell'antoniniano in particolare per lo meno per quanto attiene alle zecche italiche. Questo riguarda non solo il contenuto argenteo che continua a depauerarsi nella V (266) e nella VI (267-268) serie urbica ${ }^{39}$ e parallelamente anche a Mediolanum ${ }^{40}$, ma interessa in buona misura anche i livelli produttivi che aumentano straordinariamente come dimostra il raddoppio delle officine operanti a Roma dal 266 (da 6 a 12) ${ }^{41}$ e l'ampiamento della struttura anche nella zecca lombarda ${ }^{42}$. L'andamento inflativo è evidente e deve avere

altri, anche sulla base dei dati numismatici, andrebbe anticipato al 267 o anche prima (CHRISTOL 1975, 821825 e bibliografia ivi citata; BASTIEN 1984).

${ }^{39}$ King e Northover sospendono il giudizio sulla I serie a causa dell'esigutà dei dati disponibili. In riferimento alle serie successive, stanti i risultati delle analisi effettuate da loro stessi e da altri (LE GENTILHOMME 1962; TYLER 1975; COPE/KING/CLAY 1997), propongono uno standard del contenuto d'argento di 36 scrupoli per libbra (dal 14\% al 12,2\%) o 30 (dal 10,1\% al 11,8\%) per la II serie, di 24 scrupoli (da 9, 6 a 8\%) per la III e la IV, di 18 (da 5,9 a 7,3\%) o 15 (da 6,2 a 4,9\%) per la V, infine di solo 6 scrupoli (da 2,7 a 1,7\%) per la VI: KING/ NORTHOVER 1997, 75.

40 BESLY/BLAND 1983, 37; KING/NORTHOVER 1997, 75, 87, 99.

41 CRISAFULLI 2008, 40-41.

42 CRISAFULLI 2008, 71. 
inciso negativamente sulla percezione del valore delle monete circolanti, già di qualità pessima. Questi elementi possono dunque avere innescato tensioni alla tesaurizzazione, basate sui principi della Legge di Gresham.

D'altro canto, alcuni dei gruzzoli menzionati hanno dimensioni piuttosto rilevanti, tali da rendere naturale l'eventualità che si trattasse di casse finalizzate a impieghi di natura militare, come nel caso del più cospicuo tesoro italiano di questa fase, ossia quello di Falerone contenente circa 7600 monete $^{43}$ (Tabella 2). Le due possibilità non paiono facilmente conciliabili, ma come extrema ratio sembrerebbe più plausibile ricondurre anche $\mathrm{i}$ grandi nuclei all'ipotesi economica piuttosto che il contrario: in effetti anche 7600 antoniniani potrebbero costituire un peculio familiare. Se possiamo presumere ragioni economiche, forse connesse all'aspettativa di continue e sempre più pesanti svalutazioni dell'antoniniano, i ripostigli citati permettono di definire l'estensione geografica del fenomeno all'Italia centro-settentrionale, dove la moneta aveva evidentemente maggiori possibilità di circolare in relazione a una maggiore presenza militare a scopi difensivi.

Queste considerazioni rendono di fatto plausibili tutte le aree di attività/“influenza" del Cardinale Giovan Battista Zen, così come sono state individuate sopra, quali ambiti di possibile rinvenimento del gruzzolo, che verrebbe dunque a collocarsi coerentemente in un quadro geografico e cronologico definito su altri elementi già conosciuti. Non è a nostro avviso possibile escludere alcuno degli ambiti regionali individuati su queste basi,

\footnotetext{
43 Tale ipotesi è stata presa in considerazione anche per la fase gallienica in riferimento al ripostiglio di Ceraino (MORATI 1971, 61-69; RMRVe, III/3, 13/1(2)), in alcune fonti detto terminare con Valeriano ed essere composto da molto più di 10000 esemplari, e rapportandolo con i gruzzoli molto cospicui di alcune fasi successive, come quello della Venera, terminante con monete della tetrarchia: GORINI 1987, 263. In realtà una recente revisione del rispostiglio di Ceraino dimostra che questo temina in età gallienica e, pur contenendo varie centinania di esemplari, non superava il migliaio: CRISAFULLI c.s. Va dunque riconsiderata quella ipotesi alla luce di queste nuove scoperte.
}

né pare discriminante in questo senso la presenza di zecche non italiche nel gruzzolo del Cardinale. Accanto alle principali officine italiche (Roma e Mediolanum), sono attestati infatti numerosi atelier monetari orientali, certamente attribuibili, distribuiti tra l'epoca di Settimio Severo e quella di Treboniano Gallo ${ }^{44}$; peraltro, compaiono con una certa frequenza anche la zecca di Mediolanum e quella di Viminacium ${ }^{45}$, mentre altre zecche occidentali (Treviri?) risultano presenti sicuramente solo con una moneta. Tale varietà di fonti di approvvigionamento monetario si ritrova in molti dei ripostigli su ricordati, a cominciare dai più cospicui come per esempio Torino, San Michele in Lodivecchio, Grumello, Falerone, per continuare con quelli omologhi per dimensione, come per esempio Ceretto Lomellina, Gattinara, Pombia e Ostia III, senza una evidente limitazione geografica (Tabella 2); nei ripostigli di dimensioni ridotte, con poche decine di esemplari, queste presenze sono meno percepibili. Tale ricorrenza sembra alludere a un fenomeno di ampia portata che non fa presagire specifiche circostanze contingenti, ma sembra indicare una diffusione più generalizzata, forse da riconnettere con spostamenti di truppe dall'Oriente anche di molto precedenti alla

\footnotetext{
44 Settimio Severo, Emesa: Cartella A, n. 5; Gordiano III, Antiochia: Cartella B, nn. 7-8, 34, 69-71; Treboniano Gallo, Antiochia: Cartella D, nn. 2, 5.

45 L'attribuzione a questa zecca di serie ascrivibili a Valeriano, Gallieno e Salonina è proposta in FITZ 1978, part. 665-680 e rimane la più accettata, pur non essendo unanimente condivisa. In tal senso v. Carson il quale sostiene che anche "Le Gentilhomme's suggestion Aquileia merits consideration": CARSON 1978, 73 L'autore francese riteneva più probabile la localizzazione dell'emissione di queste serie ascrivibili genericamente a una "seconda" zecca occidentale in Italia nordorientale piuttosto che a Viminacium: questa seconda zecca occidentale, infatti, era certamente attiva già a partire dalle fasi iniziali del regno di Valeriano, ossia quando quest'ultimo governava da solo senza Gallieno. In questa frazione cronologica la Mesia sarebbe stata sotto il controllo militare di Emiliano e sarebbe stato pertanto piuttosto improbabile che Valeriano avesse potuo aprirvi un nuovo atelier. Dovendo quindi optare per un altro centro, lo studioso francese proponeva Aquileia "un noeud de routes importantes e le grand port de l'Adriatic" (LE GENTILHOMME 1946, 65).
} 
fase gallienica.

In buona sostanza gli ambiti veneto, romagnolo e laziale rimangono tutti probabili per collocare il rinvenimento del gruzzolo del Cardinale. Semmai l'incidenza della zecca di Mediolanum, poco evidente in questo contesto, similmente a ripostigli laziali e diversamente dai tesori lombardi (cfr. Tabella 2), potrebbe indurre a preferire l'ultima tra le tre aree.

\section{APPENDICE}

Nella tabella che segue viene riportata la trascrizione integrale del catalogo relativo al contenuto della Cassella II dello Scrigno D già Morosini, presente nel manoscritto su citato BMVe, mss. it., Cl. XI, n. 330 (=7140), nel quale si può identificare, come detto, l'elenco delle monete appartenute al Cardinale Giovan Battista Zen; le cancellazioni a inchiostro, i punti di sospensione e le sottolineature sono nel testo originale e vengono riprodotte integralmente. Nelle colonne III e IV, evidenziate con una differente cornice, sono inseriti elementi non contenuti in questo testo, ossia rispettivamente la classificazione delle monete e l'indicazione della raccola museale dove attualmente si trovano, seguita dai dati tecnici (peso, diametro massimo, andamento degli assi) relativi a ciascun pezzo.

Per la classifficazione generalmente viene impiegato $R I C$, tranne in casi particolari debitamente segnalati; oltre al numero di RIC in questa colonna sono riportati: prima del numero stesso, il nominale siglato (D) solo nel caso si tratti di denari, mentre gli antoniniani non sono segnalati in alcun modo; dopo il numero stesso, la zecca siglata (Tre = Treviri; $\mathrm{Rm}=$ Roma; Med = Mediolanum; $\mathrm{Vm}=$ Viminacium; Cyz = Cyzicus; Ant = Antiochia; $\mathrm{Em}=$ Emesa), la cronologia tra parentesi tonde; queste due ultime informazioni non rispondono necessariamente alle indicazioni proposte in RIC, specie per le emissioni di Valeriano e Gallieno, ma sono aggiornate in base alla letteratura più recente, particolarmente ELMER 1941, GÖBL 1951, CARSON 1967-1968, ELKS 1972, FITZ 1978, BESLY/BLAND 1983, MIR, 36;
Rimane però il fatto che, proprio in relazione alle fasi in cui l'atelier lombardo iniziò ad operare, i dati di cui disponiamo sono meno affidabili e non permettono di discriminare la reale incidenza di questa fabbrica monetaria. Ci sembra più opportuno dunque sospendere il giudizio, per lo meno finché non si riuscirà a disporre di nuove informazioni sui destini del materiale attualmente irreperibile.

solo nel caso delle monetazioni di Gallieno e di Salonina il numero di RIC può essere preceduto da un asterisco $\left(^{*}\right)$, cui seguono le coniazioni pertinenti alla fase successiva alla morte di Valeriano. Sono classificati tutti gli esemplari reperiti presso le due raccolte museali; inoltre, ove il livello di dettaglio delle descrizioni manoscritte lo permetta, sono catalogate anche le monete attualmente non più conservate nei due Musei: in questi casi, il trattino (-) nell'ultima colonna ribadisce che il pezzo corrispondente è irreperibile. Qualora tra le monete attualmente conservate vi siano esemplari rispondenti alla medesima classificazione o descrizione nell'ambito delle diverse autorità emittenti, i quali a loro volta corrispondano nel manoscritto a elencazioni di pezzi simili tra loro, la corrispondenza tra le monete oggi presenti e quelle elencate è del tutto arbitraria e risponde unicamente alla provenienza museale, facendo precedere quelle del Museo Archeologico Nazionale, e al numero d'inventario dell'ente proprietario.

Per individuare la raccolta museale si utilizzanola lettera A seguita da un numero, per specificare il Museo Archeologico Nazionale di Venezia e il numero d'inventario, e la lettera B seguita da un numero, per indicare il Museo Correr di Venezia e il numero d'inventario. Tutte le monete reperite nelle due collezioni sono riprodotte in fotografia in scala naturale: la numerazione delle foto corrisponde a quella del catalogo manoscritto, rispettando la suddivisione in base alle "cartelle" (A, B, $C, D)$ e alla progressione numerica che inizia nuovamente per ciascuna di queste. 


\begin{tabular}{|c|c|c|c|}
\hline & & nominale/RIC/zecca/(data) & Collocazione \\
\hline \multicolumn{4}{|l|}{ [p.] 1.} \\
\hline \multicolumn{4}{|l|}{$\begin{array}{l}\text { Cassella II. } \\
\text { Cartella A }\end{array}$} \\
\hline & $==$ Septimius Severus $==$ & & \\
\hline (argento) & $\begin{array}{l}\text { 1. Severus Pius. Caput Sept. Severi laureatum } \\
\text { R}^{\circ} \text { Cos. III. P. P. P. M. Tr. P. XIII. Figura stans. }\end{array}$ & D 196-198 Rm (205) & - \\
\hline $\begin{array}{l}\text { idem Sep.S. } \\
\text { (argento) }\end{array}$ & $\begin{array}{l}\text { 2. Caput Severi laureatum. } \\
\mathrm{R} \% \text { Marti pacifero. Mars galeatus stans, d. ramum } \\
\text { fortasse oleae, sin. hastam inversam. ab parte sin. } \\
\text { dextera cippo imposito. }\end{array}$ & D 113 Rm (197-198) & $\begin{array}{l}\text { A901 } \\
\text { g 3,39; mm 17; h } 12\end{array}$ \\
\hline $\begin{array}{l}\text { idem } \\
\text { (argento) }\end{array}$ & $\begin{array}{l}\text { 3. Caput Severi laureatum } \\
\mathrm{R}^{\circ} \text {. Munificentia Augusti - Elephas. }\end{array}$ & $\begin{array}{l}\text { D 82, } 100 \mathrm{Rm}(196- \\
197)\end{array}$ & - \\
\hline $\begin{array}{l}\text { idem } \\
\text { (argento) }\end{array}$ & $\begin{array}{l}4 \text { Caput Severi laureatum } \\
\mathrm{R} \% \text { Victoria Augusti. Victoria gradiens, d. coronam. }\end{array}$ & $\begin{array}{l}\text { D } 86(?) \mathrm{Rm}(196- \\
197)\end{array}$ & $\begin{array}{l}\text { A7038 } \\
\text { g 2,95; mm 23; h } 6\end{array}$ \\
\hline $\begin{array}{l}\text { idem } \\
\text { (argento) }\end{array}$ & $\begin{array}{l}\text { 5. Caput Sept. Severi laureatum } \\
\mathrm{R}^{\circ} \text {. T..... Trophaeum duobus captivis assidentibus. }\end{array}$ & D 389 Em (194-195) & $\begin{array}{l}\text { A7036 } \\
\text { g 3,49; mm 17; h } 12\end{array}$ \\
\hline $\begin{array}{l}\text { idem } \\
\text { (argento) }\end{array}$ & $\begin{array}{l}\text { 6. Caput ut supra } \\
\text { R\% . Apollini Augusto. dext. pateram, sin. Lyram. }\end{array}$ & D 40 Rm (194-195) & ( \\
\hline $\begin{array}{l}\text { idem } \\
\left(\arg ^{\text {to }}\right)\end{array}$ & 7. Nummus similis n. ${ }^{\circ}$. & $\begin{array}{l}\text { D 113, } 134 \text { Rm (197- } \\
200)\end{array}$ & - \\
\hline $\begin{array}{l}\text { idem } \\
\left(\text { arg. }^{\text {to }}\right)\end{array}$ & $\begin{array}{l}\text { 8. Caput ut supra. } \\
\mathrm{R} \% \text {. Iovi fotrasse propugnatori. Juppiter stans } \\
\text { manibus, ut videtur, extensis. }\end{array}$ & D 131 Rm (198-200) & $\begin{array}{l}\text { A7037 } \\
\text { g 2,93; mm 17; h } 6\end{array}$ \\
\hline $\begin{array}{l}\text { idem } \\
\left(\text { arg. }^{\text {to }}\right)\end{array}$ & $\begin{array}{l}\text { 9. Caput ut supra } \\
\text { R\% Annona Aug; Mulier stans, dext. demissa, sin. } \\
\text { cornucopiae. }\end{array}$ & D 107 Rm (197-198) & $\begin{array}{l}\text { A878 } \\
\text { g } 3,04 ; \mathrm{mm} 17 ; \mathrm{h} 1\end{array}$ \\
\hline [p.] 2. & $==\underline{\text { Julia Pia }}==$ & & \\
\hline$\left(\arg ^{\text {to }}\right)$ & $\begin{array}{l}\text { 10. Julia Pia felix Augusta. Caput ejusdem } \\
\text { R\% . Diana Lucifera Stans. }\end{array}$ & $\begin{array}{l}\mathrm{D} 373 \mathrm{~A} \mathrm{Rm} \quad(211- \\
217)\end{array}$ & $\begin{array}{l}\text { A920 } \\
\text { g 3,19; mm 18; h } 1\end{array}$ \\
\hline $\begin{array}{l}\text { eadem } \\
\left(\arg .^{\text {to }}\right)\end{array}$ & $\begin{array}{l}11 \text { Caput ut supra. } \\
\mathrm{R} \% \text { Pietas Augusta. Mulier cum patera sacrificans } \\
\text { ad aram. }\end{array}$ & D 572 Rm (196-211) & $\begin{array}{l}\text { A924 } \\
\text { g 3,25; mm 19; h } 6\end{array}$ \\
\hline $\begin{array}{l}\text { eadem } \\
\left(\arg .^{\text {to }}\right)\end{array}$ & $\begin{array}{l}12 \text { Caput ut supra. } \\
\mathrm{R} \% \text {...Figura stans } d . \text { pateram, sin. hastam, ad } \\
\text { pedes.... }\end{array}$ & D 587 Rm (196-211) & $\begin{array}{l}\text { A7042 } \\
\text { g 3,20; mm 16; h } 6\end{array}$ \\
\hline $\begin{array}{l}\text { eadem } \\
\left(\arg .^{\text {to }}\right)\end{array}$ & $\begin{array}{l}13 \text { Caput ejusdem } \\
\mathrm{R}^{\circ} \% \text { fortasse Venus felix, } \underline{\mathrm{d} . \text { pomum, }} \text { sin. peplum. }\end{array}$ & D 580 Rm (196-211) & $\begin{array}{l}\text { A7040 } \\
\text { g } 2,49 ; \mathrm{mm} 17 ; \mathrm{h} 6\end{array}$ \\
\hline \multirow[t]{2}{*}{$\begin{array}{l}\text { eadem } \\
\left(\text { arg. }{ }^{\text {to }}\right)\end{array}$} & $\begin{array}{l}\text { 14. Caput ut supra } \\
\mathrm{R}^{\circ} \% \text {...Felix. Mulier Stans, d. ..., sin. ... }\end{array}$ & D 580 Rm (196-211) & $\begin{array}{l}\text { A7041 } \\
\text { g 2,94; mm 19; h } 12\end{array}$ \\
\hline & $==\underline{\text { Caracalla }}==$ & & \\
\hline$\left(\arg .^{\text {to }}\right)$ & $\begin{array}{l}\text { 15. Imp. Caes. M. Aur. Antoninus Augustus. Caput } \\
\text { coronatum. } \\
\mathrm{R}^{\circ} \% \text { Mars Victor armatus gradiens. }\end{array}$ & $\begin{array}{l}\mathrm{D} 10(?) \mathrm{Rm}(196- \\
198 ?)\end{array}$ & - \\
\hline $\begin{array}{l}\text { idem } \\
\left(\arg .^{\text {to }}\right)\end{array}$ & $\begin{array}{l}\text { 16. Caput ejusdem nudum. } \\
\mathrm{R}^{\circ} / \text { Marti Victori. Mars armatus gradiens. }\end{array}$ & D 11 Rm (196-198?) & $\begin{array}{l}\text { A7068 } \\
\text { g 3,40; mm 18; h } 12\end{array}$ \\
\hline $\begin{array}{l}\text { idem } \\
\left(\arg \cdot^{\text {to }}\right)\end{array}$ & $\begin{array}{l}\text { 17. Caput ejusdem laureatum. } \\
\mathrm{R}^{\circ} \% \text { Rector Orbis. Juppiter Stans d. globum, sin. hastam. }\end{array}$ & D 141 Rm (201-206) & \\
\hline $\begin{array}{l}\text { idem } \\
\left(\arg .^{\text {to }}\right)\end{array}$ & $\begin{array}{l}\text { 18. Caput ut supra. } \\
\mathrm{R}^{\circ} \text {. Pontif. Tr. P. XII. Cos. III. Figura militaris d. pileum, } \\
\text { sin. scipionem militarem, sinistrum pedem elevans. }\end{array}$ & D 112 Rm (209) & $\begin{array}{l}\text { A959 } \\
\text { g 2,91; mm 19; h } 12\end{array}$ \\
\hline
\end{tabular}




\begin{tabular}{|c|c|c|c|}
\hline & & nominale/RIC/zecca/(data) & Collocazione \\
\hline $\begin{array}{l}\text { idem } \\
\left(\text { arg. }^{\text {to }}\right)\end{array}$ & $\begin{array}{l}19 \text { Caput ut supra. } \\
\text { R\% P. M. Tr. P. II. Cos. II. Figura militaris stans d. elata, } \\
\text { sin. scipionem ad pedes fortasse captivis assidentibus }\end{array}$ & D 96 Rm (207) & - \\
\hline [p.] 3. & $==\underline{\text { Sequitur Caracalla }==}$ & & \\
\hline$\left(\arg \cdot{ }^{\text {to }}\right)$ & $\begin{array}{l}20 \text { Caput laureatum. } \\
\text { R}^{\circ} \text { P. M. Tr. P. II. Cos. III. P. P. Figura militaris d. elata } \\
\text { pede globo imposito. }\end{array}$ & D 95 (?) Rm (207) & - \\
\hline $\begin{array}{l}\text { idem } \\
(\text { arg.to })\end{array}$ & $\begin{array}{l}21 \text { Caput laureatum. } \\
\mathrm{R}^{\circ} \% \text { [testo cancellato illeggibile, n.d.r.] ---III Mulier } \\
\text { stans d. ..., sin. cornucopiae. }\end{array}$ & $\mathrm{D} ?$ & - \\
\hline $\begin{array}{l}\text { idem } \\
\text { (d'arg.to })\end{array}$ & $\begin{array}{l}\text { 22. Antoninus Pius Augustus. Caput laureatum. } \\
\mathrm{R}^{\circ} \text {. Virtus Augusti. Figura galeata stans, dext. } \\
\text { victoriolam, sin. hastam. }\end{array}$ & $\begin{array}{l}\text { D 147-149 Rm (201- } \\
\text { 206) }\end{array}$ & \\
\hline \multirow[t]{3}{*}{$\begin{array}{l}\text { idem } \\
\left(\text { arg.t }^{\text {to }}\right)\end{array}$} & $\begin{array}{l}23 \text { Caput laureatum. } \\
\text { R\% } \% \text {. M. Tr. P. XVI. Cos. III. Figura stans dext. elata, } \\
\text { sin. longum scipionem. }\end{array}$ & D $208 \mathrm{Rm}(213)$ & - \\
\hline & [testo cancellato illeggibile, $n . d . r$. ] & & \\
\hline & $==\underline{\text { Septim. Geta }}==$ & & \\
\hline$\left(\right.$ arg.to ${ }^{\text {to }}$ & $\begin{array}{l}24 \text { Sept. Geta Caes. Caput Septimii leureatum. } \\
\text { R\% . Font. [sic!] Tr. P. Cos. Pallas stans, d. scuto } \\
\text { innixa, sin. hastam. }\end{array}$ & $\begin{array}{l}\text { D } 34(?) \mathrm{Rm}(203- \\
\text { 208) }\end{array}$ & - \\
\hline $\begin{array}{l}\text { idem } \\
\left(\text { arg.t }{ }^{\circ}\right)\end{array}$ & 25 Nummus similis superiori. & c.s. & - \\
\hline \multirow[t]{2}{*}{$\begin{array}{l}\text { idem } \\
\left(\text { arg.t }{ }^{\circ}\right)\end{array}$} & $\begin{array}{l}\text { 26. Caput Getae nudum. } \\
\text { R\% \% [testo cancellato illeggibile, } n . \text { d.r.] Pon. Tr. P. Cos. } \\
\text { II. Figura stans d. ...sin scipionem. }\end{array}$ & $\begin{array}{l}\text { D RIC pp. 323-325 } \\
\operatorname{Rm}(208-210)\end{array}$ & - \\
\hline & $==\underline{\text { Elagabalus }}==$ & & \\
\hline$\left(\arg .^{\mathrm{to}}\right)$ & $\begin{array}{l}\text { 27. Imp. Antoninus Pius Aug. Caput Elagabali laur. } \\
\mathrm{R}^{\circ} \% \text { Libertas Aug. Figura stans, d. pileum, sin. baculum }\end{array}$ & $\begin{array}{l}\text { D 106-108 Rm (218- } \\
\text { 222) }\end{array}$ & - \\
\hline $\begin{array}{l}\text { idem } \\
\left(\text { arg.t }{ }^{\circ}\right)\end{array}$ & $\begin{array}{l}28 \text { Caput laureatum. } \\
\text { R\%. P. M. Tr. P. Cos. III. P. P. Figura sacrificans cum } \\
\text { patera ad aram etc. in area sol. }\end{array}$ & D 46d Rm (221) & $\begin{array}{l}\text { B4101 } \\
\text { g 2,10; mm 19,5; h } \\
12\end{array}$ \\
\hline [p.] 4 & $==$ Sequitur Elagabalus $==$ & & \\
\hline$\left(\arg .^{\text {to }}\right)$ & $\begin{array}{l}29 \text { Caput ejusdem laureatum. } \\
\mathrm{R}^{\circ} / \ldots \ldots . . \text { Figura radiata stans; d. ..., sin. hastam. }\end{array}$ & \begin{tabular}{|l|}
$\mathrm{D} 17,28,38,40 \mathrm{Rm}$ \\
$(218-221)$
\end{tabular} & - \\
\hline \multirow[t]{2}{*}{$\begin{array}{l}\text { idem } \\
\left(\text { arg.t }{ }^{\mathrm{to}}\right)\end{array}$} & $\begin{array}{l}30 \text { Caput laureatum. } \\
\mathrm{R}^{\circ} \% \text { Summus Sacerdos Aug. Figura sacrificans ad } \\
\text { aram supra quam Sol. }\end{array}$ & D 146 Rm (218-222) & - \\
\hline & $==$ Julia Paula $==$ & & \\
\hline \multirow[t]{2}{*}{$\left(\arg .^{\text {to }}\right)$} & $\begin{array}{l}31 \text { Julia Paula Aug. Caput illius. } \\
\text { R}^{\circ} / \text { Concordia. Figura sedens, d. pateram supra stella. }\end{array}$ & D $211 \mathrm{Rm}(218-222)$ & - \\
\hline & $==$ Julia Maesa $==$ & & \\
\hline$\left(\arg .^{\mathrm{to}}\right)$ & $\begin{array}{l}\text { 32. Julia Maesa Aug. Jul. Maesae. Caput Juliae Mesae. } \\
\text { R\%. Pudicitia. Figura sedens. }\end{array}$ & D $268 \mathrm{Rm}(218-222)$ & $\begin{array}{l}\text { A1058 } \\
\text { g 2,90; mm 19; h } 6\end{array}$ \\
\hline $\begin{array}{l}\text { eadem } \\
\left(\text { arg.t }{ }^{\circ}\right)\end{array}$ & $\begin{array}{l}33 \text { Caput ut supra. } \\
\mathrm{R}^{\circ} \% \text { Fortasse Juno. Figura stolata d. pateram, sin. } \\
\text { hastam. }\end{array}$ & $\begin{array}{l}\text { D 259-260 Rm (218- } \\
\text { 222) }\end{array}$ & - \\
\hline $\begin{array}{l}\text { eadem } \\
(\text { arg.t. }\end{array}$ & $\begin{array}{l}34 \text { Caput ut supra. } \\
\mathrm{R} \% \text { Pudicitia. Figura velata sedens, d. ori admovens } \\
\text { sin. hastam. }\end{array}$ & D $268 \mathrm{Rm}(218-222)$ & - \\
\hline
\end{tabular}




\begin{tabular}{|c|c|c|c|}
\hline & & nominale/RIC/zecca/(data) & Collocazione \\
\hline $\begin{array}{l}\text { eadem } \\
\left(\text { arg. }{ }^{\circ}\right)\end{array}$ & 35. Nummus fere similis superiori. & c.s. & - \\
\hline $\begin{array}{l}\text { eadem } \\
(\text { arg.t. }\end{array}$ & $\begin{array}{l}36 \text { Caput ut supra. } \\
\mathrm{R} \% \text { Saeculi Felicitas. Figura stans, d. pateram super } \\
\text { aram, sin. Caduceum longum. }\end{array}$ & $\begin{array}{l}\text { D 271-272 Rm (218- } \\
\text { 222) }\end{array}$ & - \\
\hline [p.] 5 & $==\underline{\text { M. Aurelius Alexander }}==$ & & \\
\hline$\left(\right.$ arg.t $\left.{ }^{\text {to }}\right)$ & $\begin{array}{l}37 \text { Imp. C. M. Aur. Severus Alexander Aug. Caput } \\
\text { Alexandri Severi. } \\
\text { R\% Virtus Aug. Figura militaris stans, d. hastam s. } \\
\text { scuto imposito, et pede sinistro elev. }\end{array}$ & $\begin{array}{l}\text { D 222, } 259 \mathrm{Rm}(228- \\
\text { 235) }\end{array}$ & - \\
\hline $\begin{array}{l}\text { idem } \\
(\text { arg.to })\end{array}$ & $\begin{array}{l}38 \text { Caput ut supra } \\
\text { R\%. P. M. Tr. P. XII. Cos. III P. P. Figura stans, d. elata, } \\
\text { sin. fortasse scipionem. }\end{array}$ & D 120 Rm (233) & - \\
\hline $\begin{array}{l}\text { idem } \\
\left(\text { arg.t }^{\text {to }}\right)\end{array}$ & $\begin{array}{l}39 \text { Caput ut supra. } \\
\mathrm{R}^{\circ} \text {. Multier Providentia. Mulier stans, d. spicas } \\
\text { super modium, sin. ..., in area ... }\end{array}$ & $\begin{array}{l}\text { D 252b Rm (231- } \\
\text { 235) }\end{array}$ & $\begin{array}{l}\text { A1067 } \\
\text { g } 2,90 ; \mathrm{mm} \mathrm{19;} \mathrm{h} 12\end{array}$ \\
\hline $\begin{array}{l}\text { idem } \\
(\text { arg.t. }\end{array}$ & $\begin{array}{l}\text { 40. Caput ut supra } \\
\text { R०\%. P. M. Tr. II. Cos. ... Figura stans, d. extensa, sin. } \\
\text { hastam. }\end{array}$ & D 37 Rm (224) & $\begin{array}{l}\text { B4284 } \\
\text { g 2,68; mm 19; h } 1\end{array}$ \\
\hline$\frac{\text { idem }}{\left(\text { arg.t }{ }^{\text {to }}\right)}$ & $\begin{array}{l}41 \text { Caput ut supra } \\
\mathrm{R} \% \text {. Felicitas Aug. Mulier stans d. pateram super } \\
\text { aram, sin. cornucopiae. }\end{array}$ & $\begin{array}{l}\mathrm{D} \text { 137, } 192 \mathrm{Rm}(222- \\
\text { 231) }\end{array}$ & - \\
\hline $\begin{array}{l}\text { idem } \\
\left(\text { arg.t }{ }^{\text {to }}\right)\end{array}$ & $\begin{array}{l}42 \text { Caput ut supra. } \\
\mathrm{R} \% \text { Jovi Statori. Juppiter stans, d. hastam. }\end{array}$ & D 202 Rm (228-231) & $\begin{array}{l}\text { B4241 } \\
\text { g 4,01; mm 20; h } 6\end{array}$ \\
\hline $\begin{array}{l}\text { idem } \\
\left(\text { arg. }^{\text {to }}\right)\end{array}$ & $\begin{array}{l}\text { 43. Caput ut supra } \\
\text { R\% . P. M. Tr. P. Cos. P. P. Figura sedens dext. ...sin. } \\
\text { sellae innixa. }\end{array}$ & D 14 Rm (222) & $\begin{array}{l}\text { B4267 } \\
\text { g } 3,61 ; \mathrm{mm} \mathrm{19,5,} \mathrm{h} \\
12\end{array}$ \\
\hline $\begin{array}{l}\text { idem } \\
\left(\text { arg. }^{\text {to }}\right)\end{array}$ & $\begin{array}{l}44 \text { Caput ut supra } \\
\mathrm{R}^{\circ} \% \text {...Po:... Figura stans. }\end{array}$ & D 127 Rm (222-228) & $\begin{array}{l}\text { B4222 } \\
\text { g 2,90; mm 20; h } 6\end{array}$ \\
\hline $\begin{array}{l}\text { idem } \\
\left(\text { arg. }^{\text {to }}\right)\end{array}$ & $\begin{array}{l}45 \text { Caput ut supra. } \\
\text { R\%. P. M. Tr. P. III Cos. P. P. Figura stans, d. pateram } \\
\text { super aram. }\end{array}$ & D 50 Rm (225) & $\begin{array}{l}\text { B4279 } \\
\text { g 3,33; mm 20; h } 12\end{array}$ \\
\hline [p.] 6. & $==\underline{\text { Sequitur Alex Severus }}==$ & & \\
\hline$\left(\arg .^{\text {to }}\right)$ & $\begin{array}{l}\text { 46. Caput ut supra. } \\
\text { R०/ P. M. Tr. Pot. X... Figura gradiens dext. extensa, } \\
\text { s. scipionem. }\end{array}$ & D 120 Rm (233) & $\begin{array}{l}\text { A1060 } \\
\text { g 3,35; mm 21; h } 12\end{array}$ \\
\hline $\begin{array}{l}\text { idem } \\
\left(\text { arg.t }{ }^{\circ}\right)\end{array}$ & $\begin{array}{l}\text { 47. Caput ut supra. } \\
\text { R\% . Libertas Aug. Mulier stans, d. pileum, sin. } \\
\text { columnae innixa. }\end{array}$ & $\begin{array}{l}\mathrm{D} \text { 155(?) } \mathrm{Rm}(222- \\
228)\end{array}$ & - \\
\hline $\begin{array}{l}\text { idem } \\
\left(\text { arg.t }{ }^{\circ}\right)\end{array}$ & $\begin{array}{l}\text { 48. Caput ut supra. } \\
\text { R\%.Victoria Aug. Victoriam [sic!] coronam protendens. }\end{array}$ & $\begin{array}{l}\mathrm{D} 180,212,215,258 \\
\operatorname{Rm}(222-235)\end{array}$ & - \\
\hline \multirow[t]{2}{*}{$\begin{array}{l}\text { idem } \\
\left(\text { arg. }^{\text {to }}\right)\end{array}$} & $\begin{array}{l}\text { 49. Caput ut supra. } \\
\mathrm{R} \% \text { Romae Aeternae. Figura sedens victoriolam } \\
{[\text { sic!], sin. hastam. }}\end{array}$ & D 176 Rm (222-228) & - \\
\hline & $==\underline{\text { Julia Mamaea Aug. }==}$ & & \\
\hline$\left(\arg .{ }^{\text {to }}\right)$ & $\begin{array}{l}\text { 50. Julia Mamaea Augusta. Caput illius. } \\
\text { R\%/ Fecund. Augustae. Mulier sedens puerulo adstante, } \\
\text { cui pateram porrigere videtur, sin. hastam. }\end{array}$ & D 331 Rm (222-235) & - \\
\hline$\frac{\text { eadem }}{\left(\text { arg. } .^{\text {to }}\right)}$ & $\begin{array}{l}\text { 51. Caput ut supra. } \\
\mathrm{R} \% \text { Vesta. Figura stans, d. palladium, sin. hastam }\end{array}$ & D 360 Rm (222-235) & $\begin{array}{l}\text { B4484 } \\
\text { g 2,65; mm 21; h } 6\end{array}$ \\
\hline
\end{tabular}




\begin{tabular}{|c|c|c|c|}
\hline & & nominale/RIC/zecca/(data) & Collocazione \\
\hline $\begin{array}{l}\text { eadem } \\
\left(\text { arg. }{ }^{\mathrm{t}}\right)\end{array}$ & $\begin{array}{l}\text { 52. Caput ut supra. } \\
\mathrm{R}^{\circ} \text {. Vesta. F.ra similis superiori. }\end{array}$ & c.s. & $\begin{array}{l}\text { B4485 } \\
\text { g 2,80; mm 19; h } 12\end{array}$ \\
\hline$($ arg.to $)$ & $\begin{array}{l}\text { 53. Caput ut supra. } \\
\mathrm{R}^{\circ} \% \text { Juno Conservatrix. Figura stans, d. pateram, ad } \\
\text { pedes Pavo, sin. hastam. }\end{array}$ & D 343 Rm (222-235) & $\begin{array}{l}\text { B } 4474 \\
\text { g } 3,47 ; \mathrm{mm} 19 ; \mathrm{h} 12\end{array}$ \\
\hline [p.] 7. & $\begin{array}{c}==\underline{\text { Sequitur Severus Alexander }}== \\
==\underline{\text { Sequitur Julia Mamaea }}==\end{array}$ & & \\
\hline $\begin{array}{l}\text { (arg.to } \\
\text { arg. }\end{array}$ & 54. Nummus fere similis superiori. & c.s. & $\begin{array}{l}\text { B4475 } \\
\text { g 3,38; mm 19; h } 12\end{array}$ \\
\hline \multirow[t]{2}{*}{$\begin{array}{l}(\text { arg.to } \\
(\text { arg.to }\end{array}$} & $\begin{array}{l}\text { 55. Caput Juliae Mamaeae.... } \\
\mathrm{R}^{\circ} \% \text {.... Fortasse Felicitas Publica. Mulier dext. elata } \\
\text { cum caduceo, sin. columnae innixa. }\end{array}$ & D 335 Rm (222-235) & - \\
\hline & $==\underline{\text { Jul. Verus Maximinus }}==$ & & \\
\hline$\left(\right.$ arg.t ${ }^{\text {to }}$ & $\begin{array}{l}\text { 56. Imp. Maximinus Pius Augustus. Caput laureatum. } \\
\mathrm{R} \% \text {. Victoria Aug. gradiens ad sinistram d. [testo } \\
\text { cancellato illeggibile, } n \text {.d.r.] coronam, super } \\
\text { humerum sin. ramum lauri. }\end{array}$ & D 16 Rm (235-236) & $\begin{array}{l}\text { A1141 } \\
\text { g } 2,95 ; \mathrm{mm} 21 ; \mathrm{h} 7\end{array}$ \\
\hline $\begin{array}{l}\text { idem } \\
(\text { arg.to }\end{array}$ & $\begin{array}{l}57 \text { Caput ut supra. } \\
\mathrm{R}^{\circ} \% \text { Pax Augusti. Mulier stans, d. ramum ad [sic!] } \\
\text { sin. hastam transversam. }\end{array}$ & D 12 Rm (235-236) & $\begin{array}{l}\text { B4541 } \\
\text { g 2,65; mm 19; h } 12\end{array}$ \\
\hline $\begin{array}{l}\text { idem } \\
(\text { arg.to }\end{array}$ & $\begin{array}{l}58 \text { Caput ut supra. } \\
\text { R\%. Fides Militum. Figura stans utraq. manu tenens } \\
\text { signum militare. }\end{array}$ & D 7A Rm (236-236) & $\begin{array}{l}\text { A1133 } \\
\text { g 3,05; mm 21; h } 12\end{array}$ \\
\hline $\begin{array}{l}\text { idem } \\
\left(\text { arg. }^{\mathrm{to}}\right)\end{array}$ & $\begin{array}{l}\text { 59. Caput ut supra. } \\
\mathrm{R}^{\circ} \text {. Victoria Aug. sinistrorsum gradiens, d. coronam } \\
\text { sin. palmae ramum }\end{array}$ & $\begin{array}{l}\text { D 16, } 22 \mathrm{Rm}(235- \\
\text { 238) }\end{array}$ & - \\
\hline $\begin{array}{l}\text { idem } \\
\left(\text { arg.t }^{\text {to }}\right)\end{array}$ & $\begin{array}{l}60 \text { Caput ut supra. } \\
\mathrm{R}^{\circ} \% \text { Salus publiea Augusti. Mulier sedens serpentem } \\
\text { pascit in patera. }\end{array}$ & D 14 Rm (235-236) & $\begin{array}{l}\text { A1135 } \\
\text { g 3,80; mm 20; h } 6\end{array}$ \\
\hline $\begin{array}{l}\text { idem } \\
(\text { arg.to })\end{array}$ & $\begin{array}{l}61 \text { Caput ut supra. } \\
\mathrm{R}^{\circ} \text {. Salus Augusti. Typus similis superiori. }\end{array}$ & $\begin{array}{l}\text { D 14, } 21 \mathrm{Rm}(235- \\
238)\end{array}$ & - \\
\hline [p.] 8 & $==\underline{\text { Sequitur Maximinus }}==$ & & \\
\hline$\left(\right.$ arg.to ${ }^{\text {to }}$ & $\begin{array}{l}62 \text { Caput ut supra. } \\
\mathrm{R}^{\circ} \% \text { P. M. Tr. P. Cos P. P. Figura [testo cancellato } \\
\text { illeggibile, n.d.r.] stans tenens utraq. manu signum } \\
\text { militare. }\end{array}$ & D 1 Rm (235) & - \\
\hline $\begin{array}{l}\text { idem } \\
(\text { arg.to }\end{array}$ & $\begin{array}{l}\text { 63. Caput ut supra. } \\
\text { R\% Providentia Aug. Figura stans, d. bacillum } \\
\text { deorsum tenens, sin. cornucopiae. }\end{array}$ & D 13, $20(235-238)$ & - \\
\hline $\begin{array}{l}\text { Cassella II } \\
\text { Eartella II } \\
\text { Cartella B }\end{array}$ & $==\underline{\text { Gordianus Pius }}==$ & & \\
\hline$\left(\right.$ arg.to ${ }^{\text {to }}$ & $\begin{array}{l}\text { 1. Imp. Gordianus Pius Felix Aug. Caput Gordiani } \\
\text { laureatum. } \\
\text { R\% } \% \text { Virtuti Augusti. Hercules stans, d. ad latus } \\
\text { dextrum conver., sin. clava nixus. }\end{array}$ & 95 Rm (241-243) & $\begin{array}{l}\text { B4798 } \\
\text { g } 4,81 ; \mathrm{mm} \mathrm{23;} \mathrm{h} 12\end{array}$ \\
\hline $\begin{array}{l}\text { idem } \\
\left(\text { arg. }^{\text {to }}\right)\end{array}$ & $\begin{array}{l}2 \text { nummus similis superiori. } \\
\mathrm{R}^{\circ} \%\end{array}$ & c.s. & $\begin{array}{l}\text { B4799 } \\
\text { g 4,95; mm 21; h } 6\end{array}$ \\
\hline $\begin{array}{l}\text { idem } \\
\left(\text { arg. }^{\text {.t }}\right)\end{array}$ & $\begin{array}{l}\text { 3. Nummus fere similis superiori. } \\
\mathrm{R}^{\circ} /\end{array}$ & c.s. & $\begin{array}{l}\text { B4804 } \\
\text { g 4,67; mm 22; h } 6\end{array}$ \\
\hline
\end{tabular}




\begin{tabular}{|c|c|c|c|}
\hline & & nominale/RIC/zecca/(data) & Collocazione \\
\hline $\begin{array}{l}\text { idem } \\
\text { (arg.t) }^{+t^{\prime}}\end{array}$ & $\begin{array}{l}\text { 4. Caput ut supa. } \\
\mathrm{R} \% \text {. Jovi Statori }\end{array}$ & & \\
\hline $\begin{array}{l}\text { idem } \\
\left(\text { arg.t }{ }^{\text {to }}\right)\end{array}$ & $\begin{array}{l}\text { 4. Caput ut supra } \\
\mathrm{R}^{\circ} \text { / Jovi Statori. D. hastam sin. fulmen. }\end{array}$ & 84 Rm (241-243) & $\begin{array}{l}\text { A1183 } \\
\text { g 4,70; mm 22; h } 12\end{array}$ \\
\hline $\begin{array}{l}\text { idem } \\
\left(\text { arg.t }{ }^{\mathrm{t}}\right)\end{array}$ & $\begin{array}{l}5 \text { Caput ut supra. } \\
\mathrm{R}^{\circ} \text { / Virtus August. Nummus videtur similiter n.i } 1 \text { et } 2 \text {. }\end{array}$ & 95 Rm (241-243) & - \\
\hline [p.] 9. & Sequitur Gordianus Pius & & \\
\hline$\left(\arg .{ }^{\text {to }}\right)$ & $\begin{array}{l}\text { 6. Caput ut supra. } \\
\mathrm{R}^{\circ} \% \text { Nummus similis antecedentibus. }\end{array}$ & c.s. & - \\
\hline $\begin{array}{l}\text { idem } \\
\left(\text { arg.t }{ }^{\text {to }}\right)\end{array}$ & $\begin{array}{l}\text { 7. Caput ut supra. } \\
\mathrm{R}^{\%} \text {. Liberalitas Aug. Mulier stans dext. tesseram } \\
\text { sin. cornucopiae. }\end{array}$ & 186 Ant (238-239) & - \\
\hline $\begin{array}{l}\text { idem } \\
\left(\text { arg.t }{ }^{\mathrm{t}}\right)\end{array}$ & $\begin{array}{l}8 \text { Caput ut supra. } \\
\mathrm{R}^{\%} \% \text { Aummus Postica similis antecedenti. }\end{array}$ & c.s. & - \\
\hline $\begin{array}{l}\text { idem } \\
(\text { arg.to }\end{array}$ & $\begin{array}{l}\text { 9. Caput ut supra. } \\
\mathrm{R} \% \text { Virtus Augusti. Figura armata stans, d. ramum } \\
\text { sin. hastam, ad pedes scutum. }\end{array}$ & $6 \mathrm{Rm}(238-239)$ & $\begin{array}{l}\text { B4787 } \\
\text { g 4,76; mm 21; h 7; }\end{array}$ \\
\hline $\begin{array}{l}\text { idem } \\
\left(\text { arg. }^{\mathrm{t}}\right)\end{array}$ & $\begin{array}{l}10 \text { Caput ut supra. } \\
\mathrm{R}^{\circ} \text {. Aeternitati Aug. Typus Solis, d. elata, sin globum. }\end{array}$ & 83 Rm (241-243) & $\begin{array}{l}\text { A1207 } \\
\text { g 4,50; mm 23; } 12\end{array}$ \\
\hline $\begin{array}{l}\text { idem } \\
\left(\text { arg. }{ }^{\text {to }}\right)\end{array}$ & $\begin{array}{l}\text { 11. Nummus similis superiori. } \\
\mathrm{R} \%\end{array}$ & c.s. & $\begin{array}{l}\text { A1208 } \\
\text { g 4,65; mm 22; h } 1\end{array}$ \\
\hline $\begin{array}{l}\text { idem } \\
\left(\text { arg. }^{\mathrm{t}}\right)\end{array}$ & $\begin{array}{l}\text { 12. alius similis. } \\
\mathrm{R}^{\circ} /\end{array}$ & c.s. & $\begin{array}{l}\text { A1209 } \\
\text { g 4,25; mm 23; h } 12\end{array}$ \\
\hline $\begin{array}{l}\text { idem } \\
\left(\text { arg.t }{ }^{\mathrm{t}}\right)\end{array}$ & $\begin{array}{l}\text { 13. alius similis. } \\
\mathrm{R} \%\end{array}$ & c.s. & $\begin{array}{l}\text { A1214 } \\
\text { g 3,75; mm 23; h } 6\end{array}$ \\
\hline $\begin{array}{l}\text { idem } \\
\left(\text { arg.t }{ }^{+0}\right)\end{array}$ & $\begin{array}{l}14 \text { alius similis. } \\
\mathrm{R} \%\end{array}$ & c.s. & $\begin{array}{l}\text { A1216 } \\
\text { g 4,95; mm 23; h } 6\end{array}$ \\
\hline$\frac{\text { idem }}{\left(\text { arg.t }{ }^{\circ}\right)}$ & $\begin{array}{l}\text { 15. Caput ut supra. } \\
\text { R\% . Jovi Statori. Juppiter stans, d. hastam, sin. fulmen. }\end{array}$ & $84 \mathrm{Rm}(241-243)$ & $\begin{array}{l}\text { A1184 } \\
\text { g 4,80; mm 22; h } 6\end{array}$ \\
\hline [p.] 10 & $==$ Sequitur Gordianus Pius $==$ & & \\
\hline$\left(\arg .^{\text {to }}\right)$ & $\begin{array}{l}\text { 16. Caput ut supra } \\
\mathrm{R}^{\circ} / \text { Jovi conservatori. Juppiter stans, d. fulmen, } \underline{\sin } . \\
\underline{\text { hastam. ad pedes.... }}\end{array}$ & 2 Rm (238-239) & $\begin{array}{l}\text { A1179 } \\
\text { g 4,85; mm 21; } 6\end{array}$ \\
\hline $\begin{array}{l}\text { idem } \\
\left(\text { arg.t }{ }^{\text {to }}\right)\end{array}$ & $\begin{array}{l}\text { 17. Nummus similis n. }{ }^{\circ} 10 . \text { etc. } \\
\mathrm{R}^{\circ} \%\end{array}$ & 83 Rm (241-243) & - \\
\hline $\begin{array}{l}\text { idem } \\
\left(\text { arg. }^{\mathrm{t}}\right)\end{array}$ & $\begin{array}{l}18 \text { Nummus similis n. }{ }^{\circ} 15 \text {. } \\
\mathrm{R}^{\circ} /\end{array}$ & 84 Rm (241-243) & $\begin{array}{l}\text { A1185 } \\
\text { g 4,95; mm 24; h } 6\end{array}$ \\
\hline $\begin{array}{l}\text { idem } \\
\left(\text { arg.t }{ }^{\mathrm{t}}\right)\end{array}$ & 19 alius similis. & c.s. & $\begin{array}{l}\text { A1186 } \\
\text { g 4,15; mm 23; h } 6\end{array}$ \\
\hline $\begin{array}{l}\text { idem } \\
\left(\text { arg.t }{ }^{\text {to }}\right)\end{array}$ & $\begin{array}{l}20 \text { alius similis. } \\
\mathrm{R}^{\circ} /\end{array}$ & c.s. & $\begin{array}{l}\text { A1189 } \\
\text { g 3,40; mm 22; h } 6\end{array}$ \\
\hline $\begin{array}{l}\text { idem } \\
\left(\text { arg.t }{ }^{\text {o }}\right)\end{array}$ & $\begin{array}{l}\text { 21. alius similis. } \\
\mathrm{R} \%\end{array}$ & c.s. & $\begin{array}{l}\text { A1191 } \\
\text { g 3,50; mm 22; h } 1\end{array}$ \\
\hline $\begin{array}{l}\text { idem } \\
\left(\text { arg.t }{ }^{\mathrm{t}}\right)\end{array}$ & $\begin{array}{l}\text { 22. alius similis. } \\
\mathrm{R} \%\end{array}$ & c.s. & $\begin{array}{l}\text { A1192 } \\
\text { g 5,12; mm 22; h } 12\end{array}$ \\
\hline $\begin{array}{l}\text { idem } \\
(\text { arg.to }\end{array}$ & $\begin{array}{l}\text { 23. alius similis. } \\
\mathrm{R} \%\end{array}$ & c.s. & $\begin{array}{l}\text { A1193 } \\
\text { g 4,85; mm 23; } 12\end{array}$ \\
\hline $\begin{array}{l}\text { idem } \\
\left(\text { arg.t }^{\mathrm{t}}\right)\end{array}$ & $\begin{array}{l}24 \text { alius similis. } \\
\mathrm{R}^{\circ} \%\end{array}$ & c.s. & $\begin{array}{l}\text { A1204 } \\
\text { g 3,25; mm 24; h } 1\end{array}$ \\
\hline
\end{tabular}




\begin{tabular}{|c|c|c|c|}
\hline & & nominale/RIC/zecca/(data) & Collocazione \\
\hline $\begin{array}{l}\text { idem } \\
\left(\text { arg. }^{\text {to }}\right)\end{array}$ & $\begin{array}{l}\text { 25. alius similis. } \\
\mathrm{R} \%\end{array}$ & c.s. & - \\
\hline [p.] 11. & $==$ Sequitur Gordianus Pius $==$ & & \\
\hline$\left(\arg \cdot{ }^{\text {to }}\right)$ & $\begin{array}{l}\text { 26. Caput ut supra. } \\
\mathrm{R}^{\circ} \text {. Pax Augusti. Figura stans d. ramum, sin. bacillum. }\end{array}$ & $3 \mathrm{Rm}(238-239)$ & $\begin{array}{l}\text { A1197 } \\
\text { g 5,20; mm 22; h } 12\end{array}$ \\
\hline $\begin{array}{l}\text { idem } \\
\left(\text { arg.t }{ }^{\circ}\right)\end{array}$ & $\begin{array}{l}\text { 27. Caput ut supra } \\
\left.\mathrm{R}^{\circ} \text {. Jovi Conservatori (similis n. }{ }^{\circ} 16\right)\end{array}$ & 2 Rm (238-239) & $\begin{array}{l}\text { A1182 } \\
\text { g 4,20; mm 22; h } 12\end{array}$ \\
\hline $\begin{array}{l}\text { idem } \\
\left(\text { arg.t }^{\text {to }}\right)\end{array}$ & $\begin{array}{l}\text { 28. Caput ut supra. } \\
\mathrm{R} \% \text { Virtus Augusti. Figura militaris stans, d. scuto } \\
\text { innixa, sin. hastam. }\end{array}$ & $6 \mathrm{Rm}(238-239)$ & $\begin{array}{l}\text { B4788 } \\
\text { g 4,73; mm 23; h } 6\end{array}$ \\
\hline $\begin{array}{l}\text { idem } \\
\left(\arg \cdot{ }^{+0}\right)\end{array}$ & $\begin{array}{l}29 \text { Caput ut supra. } \\
\mathrm{R} \% \text { Virtus Augusti. }\end{array}$ & & \\
\hline $\begin{array}{l}\text { idem } \\
(\text { arg.to) }\end{array}$ & $\begin{array}{l}29 \text { Caput ut supra. } \\
\mathrm{R} \% \text { Postica similis superiori. }\end{array}$ & c.s. & $\begin{array}{l}\text { B4789 } \\
\text { g 3,67; mm 21; h } 6\end{array}$ \\
\hline $\begin{array}{l}\text { idem } \\
\left(\text { arg.t }{ }^{\circ}\right)\end{array}$ & $\begin{array}{l}30 \text { similis antecedenti. } \\
\mathrm{R} /{ }^{\circ} \text {. }\end{array}$ & c.s. & $\begin{array}{l}\text { B4791 } \\
\text { g 4,31; mm 22; h } 12\end{array}$ \\
\hline $\begin{array}{l}\text { idem } \\
\left(\text { arg.t }^{\text {to }}\right)\end{array}$ & $\begin{array}{l}31 \text { Nummus similis } \mathrm{n} .{ }^{\circ} 28 \text {. } \\
\mathrm{R}^{\circ} \%\end{array}$ & c.s. & - \\
\hline $\begin{array}{l}\text { idem } \\
\left(\arg .^{\text {to }}\right)\end{array}$ & $\begin{array}{l}\text { 32. Caput ut supra } \\
\mathrm{R}^{\circ} \text { P. M. Tr. P. IIII. Cos. II. P. P. Figura sedens, dext. } \\
\text { ramum, sin. sellae innixa. }\end{array}$ & 88 Rm (241-243) & $\begin{array}{l}\text { A1220 } \\
\text { g 4,55; mm 23; h } 12\end{array}$ \\
\hline $\begin{array}{l}\text { idem } \\
\left(\text { arg. }^{\text {to }}\right)\end{array}$ & $\begin{array}{l}33 \text { Nummus similis } \mathrm{n}^{\circ} \text { 28. et aliis. } \\
\mathrm{R}^{\circ} \%\end{array}$ & $6 \mathrm{Rm}(238-239)$ & - \\
\hline$\left.\frac{\text { idem }}{(\text { arg. }}{ }^{\text {to }}\right)$ & $\begin{array}{l}\text { 34. Caput ut supra. } \\
\mathrm{R} \% \text { Oriens Aug. Solis Typus, d. elata, s. globum. }\end{array}$ & 213 Ant (242-244) & - \\
\hline [p.].12. & $==\underline{\text { Sequitur Gordianus Pius }}==$ & & \\
\hline $\begin{array}{l}\text { idem } \\
\left(\text { arg. }^{\text {to }}\right)\end{array}$ & $\begin{array}{l}35 \text { Caput ut supra. } \\
\mathrm{R}^{\circ} \% \text { Postica similis } \mathrm{n} .^{\circ} 10 \text { et aliis seqq. }\end{array}$ & 83 Rm (241-243) & - \\
\hline $\begin{array}{l}\text { idem } \\
(\text { arg.to }\end{array}$ & $\begin{array}{l}36 \text { Similis n. }{ }^{\circ} 15 . \\
\mathrm{R}^{\circ} \%\end{array}$ & 84 Rm (241-243) & - \\
\hline$\frac{\text { idem }}{(\text { arg.to }}$ & $\begin{array}{l}\text { 37. Caput ut supra. } \\
\mathrm{R} \% \text { Aequitas Aug. Mulier stans d. bilancem, sin. } \\
\text { cornucopiae. }\end{array}$ & $63 \mathrm{Rm}(240)$ & $\begin{array}{l}\text { A1172 } \\
\text { g } 3,85 ; \mathrm{mm} \mathrm{22;} \mathrm{h} 7\end{array}$ \\
\hline $\begin{array}{l}\text { idem } \\
\left(\text { arg. }^{\text {to }}\right)\end{array}$ & $\begin{array}{l}\text { 38. Similis superiori. } \\
\mathrm{R}^{\circ} /\end{array}$ & c.s. & - \\
\hline $\begin{array}{l}\text { idem } \\
\left(\arg ^{\text {to }}\right)\end{array}$ & $\begin{array}{l}39 \text { Caput ut supra. } \\
\text { R\%. P. M. Tr. P. II. Cos. P P. Mulier velata, d. pateram } \\
\text { super aram, sin. ramum demissum. }\end{array}$ & 37 Rm (240) & $\begin{array}{l}\text { A1223 } \\
\text { g 4,40; mm 23; h } 12\end{array}$ \\
\hline$\frac{\text { idem }}{\left(\text { arg. } .^{\text {to }}\right)}$ & $\begin{array}{l}\text { 40. Similis superiori. } \\
\mathrm{R}^{\circ} \%\end{array}$ & c.s. & $\begin{array}{l}\text { A1235 } \\
\text { g } 4,50 ; \mathrm{mm} 22 ; \mathrm{h} 6\end{array}$ \\
\hline$\frac{\text { idem }}{(\text { arg.to }}$ & $\begin{array}{l}41 \text { Caput ut supra. } \\
\text { R\%. P. M. Tr. P. II. Cos. P. P. Mulier stans cum patera } \\
\text { super aram, sin. bacillum demissum. }\end{array}$ & $54 \mathrm{Rm}(240)$ & $\begin{array}{l}\text { A1201 } \\
\text { g 4,70; mm 23; h } 12\end{array}$ \\
\hline $\begin{array}{l}\text { idem } \\
\left(\arg ^{\mathrm{t}}{ }^{\circ}\right)\end{array}$ & $\begin{array}{l}42 \text { Caput ut supra. } \\
\mathrm{R}^{\circ} \text {. Fortuna redux. Fortuna sedens dext. temonem } \\
\text { sin. cornucopiae; infra ad sellam rota }\end{array}$ & $143 \mathrm{Rm}(243-244)$ & $\begin{array}{l}\text { B8804 errato } \\
\text { g 4,30; } \mathrm{mm} \mathrm{22;} \mathrm{h} 6\end{array}$ \\
\hline $\begin{array}{l}\text { idem } \\
\left(\text { arg.t }^{\text {to }}\right)\end{array}$ & $\begin{array}{l}43 \text { Caput ut supra. } \\
\text { R\% P. M. Tr. P. IIII. Cos. II. P. P. Figura stans, dext. } \\
\text { hastam transversam, sin. globum. }\end{array}$ & 92 Rm (241-243) & $\begin{array}{l}\text { A1219 } \\
\text { g 4,60; mm 21; h } 6\end{array}$ \\
\hline
\end{tabular}




\begin{tabular}{|c|c|c|c|}
\hline & & nominale/RIC/zecca/(data) & Collocazione \\
\hline [p.] 13. & $==$ Sequitur Grodianus Pius $==$ & & \\
\hline $\begin{array}{l}\text { idem } \\
(\text { (arg.to })\end{array}$ & $\begin{array}{l}\text { 44. Caput ut supra } \\
\mathrm{R} \% \text { Postica similis superiori. }\end{array}$ & c.s. & $\begin{array}{l}\text { A1221 } \\
\text { g } 4,60 ; \mathrm{mm} \mathrm{21;} \mathrm{h} 6\end{array}$ \\
\hline $\begin{array}{l}\text { idem } \\
(\text { arg.to })\end{array}$ & $\begin{array}{l}\text { 45. Nummus alius similis superiori. } \\
\mathrm{R} \%\end{array}$ & c.s. & $\begin{array}{l}\text { A1232 } \\
\text { g 4,45; mm 23; h } 12\end{array}$ \\
\hline $\begin{array}{l}\text { idem } \\
\left(\text { arg.t }^{\text {to }}\right)\end{array}$ & $\begin{array}{l}\text { 46. Nummus similis n. } 26 \text {. } \\
\mathrm{R}^{\circ} \text { / }\end{array}$ & $\begin{array}{l}\text { Probabilmente il tipo } \\
\text { è stato frainteso e la } \\
\text { moneta va identifi- } \\
\text { cata con RIC } 148 \text { Rm } \\
(243-244)\end{array}$ & $\begin{array}{l}\text { B4752? } \\
\text { g } 3,74 ; \text { mm 24; h } 7\end{array}$ \\
\hline $\begin{array}{l}\text { idem } \\
(\text { arg.to }\end{array}$ & $\begin{array}{l}\text { 47. Nummus similis n. } 28 . \\
\text { R}^{\circ} \text { P. M. Tr. P. II. Cos. P. P. Mulier stans, d. ramum, } \\
\text { sin. bacillum. }\end{array}$ & 17 Rm (239) & $\begin{array}{l}\text { A1231 } \\
\text { g } 4,80 ; \mathrm{mm} \mathrm{22;} \mathrm{h} 6\end{array}$ \\
\hline $\begin{array}{l}\text { idem } \\
\left(\text { arg.t }{ }^{\mathrm{to}}\right)\end{array}$ & $\begin{array}{l}\text { 48. Caput ut supra } \\
\mathrm{R}^{\circ} \text {. Virtuti Augusti. Similis n. }{ }^{\circ} 1 \text {. }\end{array}$ & 95 Rm (241-243) & - \\
\hline $\begin{array}{l}\text { idem } \\
(\text { arg.to }\end{array}$ & $\begin{array}{l}\text { 49. Nummus similis n. }{ }^{\circ} 47 \\
\mathrm{R}^{\circ} \%\end{array}$ & 17 Rm (239) & $\begin{array}{l}\text { B4735 } \\
\text { g 2,96; mm 23; h } 6\end{array}$ \\
\hline $\begin{array}{l}\text { idem } \\
(\text { arg.to }\end{array}$ & $\begin{array}{l}\text { 50. Caput ut supra } \\
\text { R\%\%. P. M. Tr. P. II. Cos. P. P. Figura stans, d. signum } \\
\text { militare, sin. baculum. }\end{array}$ & 15 Rm (239) & $\begin{array}{l}\text { A1238 } \\
\text { g 5,15; mm 22; h } 12\end{array}$ \\
\hline $\begin{array}{l}\text { idem } \\
\left(\arg ^{\mathrm{t}}{ }^{\circ}\right)\end{array}$ & $\begin{array}{l}51 \text { Caput ut supra. } \\
\mathrm{R}^{\circ} \% \text { Victoria Aeterna. Victoria stans, d. scuto in- } \\
\text { nixa, sin. palmam. }\end{array}$ & $\begin{array}{l}154-156 \quad \mathrm{Rm} \quad(243- \\
244)\end{array}$ & - \\
\hline $\begin{array}{l}\text { idem } \\
\left(\text { arg. }^{\text {to }}\right)\end{array}$ & $\begin{array}{l}52 \text { Caput ut supra. } \\
\mathrm{R}^{\circ} \% \text {. Postica similis } n . .^{\circ} 43 \text {. }\end{array}$ & D 115 Rm (241-243) & $\begin{array}{l}\text { A1239 } \\
\text { g 3,55; mm 20; h } 12\end{array}$ \\
\hline $\begin{array}{l}\text { idem } \\
\text { (arg.to })\end{array}$ & $\begin{array}{l}\text { 53. Caput ut supra } \\
\mathrm{R} \% \text {. Felicitas Temporum. Mulier stans d. tesseram, } \\
\text { sin. cornucopiae. }\end{array}$ & 140 Rm (243-244) & $\begin{array}{l}\text { A1211 } \\
\text { g 4,35; mm 23; h } 7\end{array}$ \\
\hline $\begin{array}{l}\text { idem } \\
(\text { arg.to }\end{array}$ & $\begin{array}{l}54 \text { Nummus alius similis. } \\
\mathrm{R}^{\circ} \%\end{array}$ & $141 \mathrm{Rm}(242-244)$ & $\begin{array}{l}\text { A1215 } \\
\text { g 4,40; mm 22; h } 6\end{array}$ \\
\hline [p.] 14 & $==$ Sequitur $\underline{\text { Gordianus Pius }}==$ & & \\
\hline$\left(\arg \cdot{ }^{\text {to }}\right)$ & $\begin{array}{l}\text { 55. Caput ut supra } \\
\mathrm{R} \% \text {. Felicitas } \mathrm{R}^{\%} \text {. Securitas Perpetua. Mulier stans, } \\
\text { d. hastam, sin. columnae innixa. }\end{array}$ & 151 Rm (243-244) & $\begin{array}{l}\text { B } 4770 \\
\text { g } 4,66 ; \mathrm{mm} 22 ; \mathrm{h} 6\end{array}$ \\
\hline $\begin{array}{l}\text { idem } \\
\left(\arg ^{\text {to }}\right)\end{array}$ & $\begin{array}{l}56 \text { Caput ut supra. } \\
\text { P. M. Tr. P. v. Cos. II. P. P. Mulier sedens, d. ramum, } \\
\text { sin. sillae [sic!] innixa. }\end{array}$ & 89 Rm (241-243) & $\begin{array}{l}\text { A1225 } \\
\text { g 4,60; mm 23; h } 6\end{array}$ \\
\hline $\begin{array}{l}\text { idem } \\
(\text { arg.to })\end{array}$ & $\begin{array}{l}\text { 57. Caput ut supra. } \\
\mathrm{R} \% \text {. Romae Aeternae. Mulier sedens d. victoriolam, } \\
\text { sin. hastam. }\end{array}$ & $55 \mathrm{Rm}(240)$ & $\begin{array}{l}\text { B4761 } \\
\text { g } 4,72 ; \mathrm{mm} \mathrm{22;} \mathrm{h} 6\end{array}$ \\
\hline$\left.\frac{\text { idem }}{(\text { arg.to }}\right)$ & $\begin{array}{l}\text { 58. Caput ut supra. } \\
\mathrm{R} \% \text { Romae Aeternae. Mulier galeata sedens supra } \\
\text { globum, dext. victoriolam, sin. hastam. }\end{array}$ & $70 \mathrm{Rm}(240)$ & $\begin{array}{l}\text { B4762 } \\
\text { g 5,74; mm 22; h } 12\end{array}$ \\
\hline $\begin{array}{l}\text { idem } \\
\left(\text { arg. }^{\text {to }}\right)\end{array}$ & $\begin{array}{l}59 \text { Similis superiori. } \\
\mathrm{R}^{\circ} \%\end{array}$ & c.s. & $\begin{array}{l}\text { B4763 } \\
\text { g 5,95; mm 21; h } 12\end{array}$ \\
\hline
\end{tabular}

\footnotetext{
${ }^{46}$ Tra le monete conservate al Museo Correr di Venezia ne risulta presente una del tipo PROVID AVG, certamente pertinente al ripostiglio, la quale non compare tra quelle elencate per Gordiano III o per qualunque altra autorità emittente. Viene inserita in questa posizione del tutto arbitrariamente per la sola presenza del bastone in mano alla figura, indicato nella descrizione manoscritta della moneta.
} 


\begin{tabular}{|c|c|c|c|}
\hline & & nominale/RIC/zecca/(data) & Collocazione \\
\hline $\begin{array}{l}\text { idem } \\
\left(\arg .^{\text {to }}\right)\end{array}$ & $\begin{array}{l}60 \text { Similis superiori. } \\
\mathrm{R} \%\end{array}$ & $38,55,70 \mathrm{Rm}(240)$ & - \\
\hline $\begin{array}{l}\text { idem } \\
(\text { arg.to })\end{array}$ & $\begin{array}{l}\text { 61. Caput ut supra. } \\
\mathrm{R}^{\circ} / \text { Concordia Atug. militaris. Mulier sedens, d. } \\
\text { pateram, sin. cornucopiae. }\end{array}$ & $49,65 \mathrm{Rm}(240)$ & - \\
\hline $\begin{array}{l}\text { idem } \\
\left(\text { arg.t }{ }^{\circ}\right)\end{array}$ & $\begin{array}{l}62 \text { Caput ut supra. } \\
\mathrm{R}^{\circ} \% \text { Postica similis superiori. }\end{array}$ & c.s. & - \\
\hline$\left.\frac{\text { idem }}{(\text { arg. }}{ }^{\text {to }}\right)$ & $\begin{array}{l}63 \text { Caput ut supra } \\
\mathrm{R}^{\circ} \text { / Secutitas Publica. Mulier sedens etc. }\end{array}$ & $\begin{array}{l}\text { D } 130 \mathrm{Rm} \text { (estate } \\
\text { 241) }\end{array}$ & $\begin{array}{l}\text { B4774 } \\
\text { g 2,75; mm 21; h } 6\end{array}$ \\
\hline$\frac{\text { idem }}{\left(\arg .^{\circ}\right)}$ & $\begin{array}{l}\text { 64. Caput ut supra. } \\
\mathrm{R}^{\circ} \% \text { Virtus Aug. Vir stans d. ramum sin. hastam ad } \\
\text { pedes scutum. }\end{array}$ & $39,56,71 \mathrm{Rm}(240)$ & - \\
\hline [p.] 15 & $==\underline{\text { Sequitur Gordianus Pius }==}$ & & \\
\hline$\left(\arg .^{\text {to }}\right)$ & $\begin{array}{l}\text { 65. Caput ut supra. } \\
\text { R\%. P. M. tr. P. v. Cos. III. P. P. Figura sedens, d. } \\
\text { ramum, sin. sellae innixa. }\end{array}$ & 89 Rm (241-243) & $\begin{array}{l}\text { A1234 } \\
\text { g 3,30; mm 23; h } 6\end{array}$ \\
\hline $\begin{array}{l}\text { idem } \\
\left(\text { arg. }{ }^{\text {to }}\right)\end{array}$ & $\begin{array}{l}\text { 66. Caput ut supra. } \\
\mathrm{R} \% \text {. Nummus similis superiori. }\end{array}$ & c.s. & $\begin{array}{l}\text { A1236 } \\
\text { g 3,60; mm 22; h } 6\end{array}$ \\
\hline $\begin{array}{l}\text { idem } \\
\left(\text { arg. }{ }^{\text {to }}\right)\end{array}$ & $\begin{array}{l}67 \text { Caput ut supra } \\
\mathrm{R} \% \text { Similis superiori. }\end{array}$ & c.s. & $\begin{array}{l}\text { A1237 } \\
\text { g 4,20; mm 22; h } 1\end{array}$ \\
\hline$\left.\frac{\text { idem }}{(\text { arg. }}{ }^{\text {to }}\right)$ & $\begin{array}{l}68 \text { Caput ut supra } \\
\mathrm{R} \% \text {. Similis superiori. }\end{array}$ & c.s. & - \\
\hline $\begin{array}{l}\text { idem } \\
\text { (arg.to })\end{array}$ & $\begin{array}{l}69 \text { Caput ut supra. } \\
\text { R\%/ Fortuna Redux. Sedet, d. temonem, sin. } \\
\text { cornucopiae. }\end{array}$ & 210 Ant (242-244) & $\begin{array}{l}\text { A1177 } \\
\text { g } 4,80 ; \mathrm{mm} \mathrm{22;} \mathrm{h} 6\end{array}$ \\
\hline $\begin{array}{l}\text { idem } \\
\left(\text { arg. }^{.0}\right)\end{array}$ & $\begin{array}{l}70 \text { Similis superiori. } \\
\mathrm{R}^{\circ} /\end{array}$ & c.s. & - \\
\hline $\begin{array}{l}\text { idem } \\
(\text { arg.to }\end{array}$ & $\begin{array}{l}\text { 71. Similis superiori. } \\
\mathrm{R}^{\circ} \text {. }\end{array}$ & c.s. & - \\
\hline \multirow[t]{2}{*}{$\begin{array}{l}\text { idem } \\
\left(\arg ^{+0}\right)\end{array}$} & $\begin{array}{l}72 \text { Caput ut supra. } \\
\mathrm{R} \% \text { Fortasse Victoria Aug. Typus Victoriae stantis. }\end{array}$ & $\begin{array}{l}5 \mathrm{Rm}, 202,217 \text { Ant } \\
(238-44)\end{array}$ & - \\
\hline & // & & \\
\hline \multicolumn{4}{|c|}{$\begin{array}{l}\text { Cassella II } \\
\text { Cartella C }\end{array}$} \\
\hline [p.] 16 & $==$ Philippus Senior $==$ & & \\
\hline$\left(\arg \cdot{ }^{\text {to }}\right)$ & $\begin{array}{l}\text { 1. Imp. M. Jul. Philippus Aug. } \\
\mathrm{R}^{\circ} \text {. Annona Aug. Mulier stans d. spicas, sin. } \\
\text { cornucopiae. }\end{array}$ & 59 Rm (247-249) & $\begin{array}{l}\text { A1275 } \\
\text { g 3,45; mm 22; h } 6\end{array}$ \\
\hline $\begin{array}{l}\text { idem } \\
\left(\text { arg.t }^{\text {to }}\right)\end{array}$ & $\begin{array}{l}\text { 2. Caput ut supra. } \\
\mathrm{R} \% \text { Liberalitas Aug. II. Mulier stans d. tesseram sin. } \\
\text { cornucopiae. }\end{array}$ & 38(b) Rm (244-247) & $\begin{array}{l}\text { A1284 } \\
\text { g 4,00; mm 22; h } 6\end{array}$ \\
\hline $\begin{array}{l}\text { idem } \\
\left(\text { arg. }{ }^{\text {to }}\right)\end{array}$ & $\begin{array}{l}\text { 3. Nummus similis superiori. } \\
\mathrm{R}^{\circ} \%\end{array}$ & c.s. & $\begin{array}{l}\text { A1295 } \\
\text { g 4,60; mm 23; h } 6\end{array}$ \\
\hline $\begin{array}{l}\text { idem } \\
\left(\text { arg.t }^{\text {to }}\right)\end{array}$ & $\begin{array}{l}\text { 4. Caput ut supra. } \\
\mathrm{R} \% \text {. Fides exercitus. Quattruor signa militaria }\end{array}$ & 62 Rm (247-249) & $\begin{array}{l}\text { A1277 } \\
\text { g 4,15; mm 22; h } 6\end{array}$ \\
\hline $\begin{array}{l}\text { idem } \\
(\text { arg.t. }\end{array}$ & $\begin{array}{l}\text { 5. Caput ut supra. } \\
\mathrm{R}^{\circ} \text { / Fides Militum. Mulier stans cum duobus signis } \\
\text { militaribus. }\end{array}$ & 32(b) Rm (244-247) & $\begin{array}{l}\text { A1290 } \\
\text { g 4,40; mm 22; h } 12\end{array}$ \\
\hline $\begin{array}{l}\text { idem } \\
\left(\text { arg. }{ }^{\text {to }}\right)\end{array}$ & $\begin{array}{l}\text { 6. Caput ut supra } \\
\mathrm{R}^{\circ} \% \text { Salus Aug. Mulier stans cum serpente. }\end{array}$ & 46(b) Rm (244-247) & $\begin{array}{l}\text { B5033 } \\
\text { g 4,83; mm 23; h } 12\end{array}$ \\
\hline
\end{tabular}




\begin{tabular}{|c|c|c|c|}
\hline & & nominale/RIC/zecca/(data) & Collocazione \\
\hline $\begin{array}{l}\text { idem } \\
\left(\text { arg. }{ }^{+0}\right)\end{array}$ & $\begin{array}{l}\text { 7. Caput ut supra. } \\
\mathrm{R}^{\circ} \text {. Salus Aug. Mulier stans cum patera etc. sin. hastam }\end{array}$ & 47 Rm (244-247) & $\begin{array}{l}\text { B5031 } \\
\text { g 4,29; mm 22; h } 12\end{array}$ \\
\hline$\frac{\text { idem }}{\left(\text { arg.t }{ }^{\mathrm{t}}\right)}$ & $\begin{array}{l}\text { 8. Caput ut supra } \\
\mathrm{R}^{\circ} \% \text {....ritas. fortasse Securitas Aug. Mulier stans d. ... } \\
\text { sin. cornucopiae. }\end{array}$ & $\begin{array}{l}\text { Forse Liberalitas Aug } \\
\text { RIC 37A Rm (244- } \\
247)\end{array}$ & - \\
\hline $\begin{array}{l}\text { idem } \\
\left(\text { arg.t }{ }^{\circ}\right)\end{array}$ & $\begin{array}{l}\text { 9. Caput ut supra } \\
\mathrm{R}^{\circ} \% \text {...... Mulier stans, d. elata, sin. hastam transversam }\end{array}$ & $\begin{array}{l}\text { Forse Pax Aetern RIC } \\
\text { 40(b) Rm (244-247) } \\
\end{array}$ & - \\
\hline [p.] 17 & Sequitur Philippus Senior & & \\
\hline arg.to & $\begin{array}{l}10 \text { Caput ut supra. } \\
\mathrm{R}^{\circ} \text {. Victoria Aug. d. coronam, sin.pat palmam. }\end{array}$ & 51 Rm (244-247) & $\begin{array}{l}\text { A1281 } \\
\text { g 4,30; mm 22; h } 6\end{array}$ \\
\hline $\begin{array}{l}\text { idem } \\
\text { (arg.to })\end{array}$ & $\begin{array}{l}11 \text { Caput ut supra } \\
\mathrm{R} \% \text { Annona Aug. dext. spicas supra modium, sin. } \\
\text { cornucopiae. }\end{array}$ & 59 Rm (247-249) & $\begin{array}{l}\text { A1289 } \\
\text { g 3,85; mm 24; h } 12\end{array}$ \\
\hline $\begin{array}{l}\text { idem } \\
(\text { arg.to }\end{array}$ & $\begin{array}{l}12 \text { Caput ut supra } \\
\text { R\% } \% \text { P. M. Tr. P. IIII. Cos. II. P. P. Mulier stans d. } \\
\text { caduceum oblungum sin. cornucopiae. }\end{array}$ & 4-5 Rm (247) & - \\
\hline $\begin{array}{l}\text { idem } \\
\left(\text { arg. }{ }^{+0}\right)\end{array}$ & $\begin{array}{l}13 \text { Caput ut supra } \\
\mathrm{R}^{\circ} / \text { Postica similis } n . .^{\circ} 11 .\end{array}$ & 28(c) $\operatorname{Rm}(244.247)$ & $\begin{array}{l}\text { A1273 } \\
\text { g 3,95; mm 24; h } 6\end{array}$ \\
\hline $\begin{array}{l}\text { idem } \\
\left(\text { arg.t }^{\text {to }}\right)\end{array}$ & $\begin{array}{l}14 \text { Caput ut supra. } \\
\mathrm{R} \% \text { Similis superiori. }\end{array}$ & c.s. & $\begin{array}{l}\text { A1274 } \\
\text { g 3,75; mm 21; h } 12\end{array}$ \\
\hline $\begin{array}{l}\text { idem } \\
\left(\text { arg.t }{ }^{\text {to }}\right)\end{array}$ & $\begin{array}{l}\text { 15. Caput supra [sic!] } \\
\mathrm{R} \% \text {. Similis superiori. }\end{array}$ & c.s. & $\begin{array}{l}\text { A1287 } \\
\text { g } 4,55 ; \mathrm{mm} 23 ; \mathrm{h} 6\end{array}$ \\
\hline$\frac{\text { idem }}{\left(\text { arg. }^{\text {to }}\right)}$ & $\begin{array}{l}\text { 16. Similis superioribus. } \\
\mathrm{R}^{\circ} \text {. }\end{array}$ & $\begin{array}{l}28(c), 59 \mathrm{Rm}(244- \\
249)\end{array}$ & - \\
\hline$\frac{\text { idem }}{\left(\text { arg.t }{ }^{\text {to }}\right)}$ & $\begin{array}{l}\text { 17. Caput ut supra } \\
\mathrm{R}^{\circ} \text { Felicitas Aug. Mulier stans d. caduceum } \\
\text { oblongum, sin. cornucopiae. }\end{array}$ & $31 \mathrm{Rm}(244-247)$ & - \\
\hline $\begin{array}{l}\text { idem } \\
(\text { arg.to })\end{array}$ & $\begin{array}{l}\text { 18. Caput ut supra } \\
\mathrm{R} \% \text {. Jovi conservatori. Figura stans d. extensa sin. } \\
\text { hastam. }\end{array}$ & $\begin{array}{l}\text { Tipo non riferibile } \\
\text { a Filippo I; forse Fi- } \\
\text { lippo II RIC } 214 \mathrm{Rm} \\
(244-246)\end{array}$ & - \\
\hline$\frac{\text { idem }}{(\text { arg.to }}$ & $\begin{array}{l}19 \text { Caput ut supra. } \\
\mathrm{R} \% \text { Romae Aeternae. Mulier sedens, d. victoriolam } \\
\text { sinistra hastam. }\end{array}$ & 44(b) Rm (244-247) & $\begin{array}{l}\text { B5006 } \\
\text { g 3,97; mm 22; h } 6\end{array}$ \\
\hline [p.] 18 & $==$ Sequitur $\underline{\text { Philippus Senior }}==$ & & \\
\hline$\left(\arg \cdot{ }^{\text {to }}\right)$ & $\begin{array}{l}\text { 20. Nummus similis antecedente. } \\
\mathrm{R}^{\circ} \%\end{array}$ & c.s. & $\begin{array}{l}\text { B5007 } \\
\text { g 4,29; mm 21; h } 6\end{array}$ \\
\hline$\frac{\text { idem }}{\text { (arg.to })}$ & $\begin{array}{l}21 \text { Alius similis. } \\
\mathrm{R}^{\circ} \text {. }\end{array}$ & c.s. & $\begin{array}{l}\text { B5011 } \\
\text { g 4,86; mm 21; h } 6\end{array}$ \\
\hline $\begin{array}{l}\text { idem } \\
(\text { arg.to }\end{array}$ & $\begin{array}{l}22 \text { Alius similis. } \\
\mathrm{R}^{\circ} \text {. }\end{array}$ & c.s. & $\begin{array}{l}\text { B5013 } \\
\text { g 4,32; mm 23; h } 12\end{array}$ \\
\hline$\frac{\text { idem }}{\left(\text { arg. }^{\text {to }}\right)}$ & $\begin{array}{l}23 \text { alius similis } \\
\mathrm{R} \%\end{array}$ & c.s. & - \\
\hline$\frac{\text { idem }}{\left(\text { arg.t }{ }^{\circ}\right)}$ & $\begin{array}{l}24 \text { Similis superioribus. } \\
\mathrm{R}^{\circ} \%\end{array}$ & c.s. & - \\
\hline $\begin{array}{l}\text { idem } \\
\left.\text { (arg.t }{ }^{\text {to }}\right)\end{array}$ & $\begin{array}{l}25 \text { Similis “ } \\
\mathrm{R}^{\circ} \% \\
\end{array}$ & c.s. & - \\
\hline$\frac{\text { idem }}{\left(\text { arg. } .^{\circ}\right)}$ & $\begin{array}{l}\text { 26. Alius similis. } \\
\mathrm{R}^{\circ} \%\end{array}$ & c.s. & - \\
\hline
\end{tabular}




\begin{tabular}{|c|c|c|c|}
\hline & & nominale/RIC/zecca/(data) & Collocazione \\
\hline & 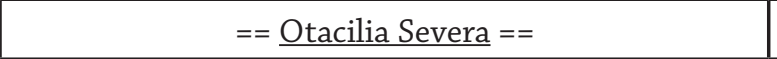 & & \\
\hline (arg. ${ }^{\text {to }}$ & $\begin{array}{l}\text { 27. Otacilia Severa Aug. Caput Otaciliae. } \\
\text { R\%. Pietas Aug. Mulier velata sacrificans ad aram. }\end{array}$ & $115 \mathrm{Rm}(248)$ & $\begin{array}{l}\text { B5144 } \\
\text { g 4,11; mm 22; h } 6\end{array}$ \\
\hline$\frac{\text { eadem }}{\text { (arg.t. }}$ & $\begin{array}{l}\text { 28. Caput ut supra. } \\
\mathrm{R} \% \text { Concordia Aug. Mulier sedens, dext. pateram, } \\
\text { sin. cornucopiae. }\end{array}$ & 125(c) Rm (246-248) & $\begin{array}{l}\text { A1331 } \\
\text { g 4,00; mm 23; h } 11\end{array}$ \\
\hline [p.] 19 & $==$ Julius Philippus Filius $==$ & & \\
\hline$($ arg.to $)$ & $\begin{array}{l}\text { 29. M. Jul. Philippus Aug. Caput Philippi Filii. } \\
\text { R\%/ Principi Juventutis. Figura militaris stans, d. } \\
\text { globum, sin. hastam. }\end{array}$ & 218(d) Rm (244-246) & $\begin{array}{l}\text { A1349 } \\
\text { g 3,40; mm 23; h } 12\end{array}$ \\
\hline $\begin{array}{l}\text { idem } \\
\text { (arg.t) }\end{array}$ & $\begin{array}{l}\text { 30. Caput ut supra. } \\
\text { Ro/ Principi }\end{array}$ & & \\
\hline$\frac{\text { idem }}{\left(\arg .{ }^{\text {to }}\right)}$ & $\begin{array}{l}\text { 30. Caput ut supra. } \\
\text { R\%/ Liberalitas Aug. III: Duae Figurae sedentes, altera } \\
\text { porrigens pateram, altera bacillum tenens }\end{array}$ & 230 Rm (247-249) & - \\
\hline $\begin{array}{l}\text { idem } \\
(\text { arg.to })\end{array}$ & $\begin{array}{l}\text { 31. Caput ut supra } \\
\mathrm{R} /{ }^{\circ} \text {. Aeternitas Imperii. Figura radiata Solis gradiens, } \\
\text { d. elevans, sinistra tenens flagrum. }\end{array}$ & 226 Rm (246-248) & $\begin{array}{l}\text { A1343 } \\
\text { g 4,75; mm 21; h } 12\end{array}$ \\
\hline$\frac{\text { idem }}{\left(\text { arg. }^{\text {to }}\right)}$ & $\begin{array}{l}\text { 32. Caput ut supra. } \\
\mathrm{R}^{\circ} \text {. Figura stans } d . \text { hastam, sin. globum. }\end{array}$ & 216(c) Rm 244-246 & $\begin{array}{l}\text { B5185 } \\
\text { g 3,80; mm 23; h } 6\end{array}$ \\
\hline $\begin{array}{l}\text { idem } \\
(\text { arg.to }\end{array}$ & $\begin{array}{l}\text { 33. Caput ut supra } \\
\mathrm{R} \% \text {. Postica similis superiori. }\end{array}$ & c.s. & - \\
\hline$\frac{\text { idem }}{\left(\text { arg. }^{\text {to }}\right)}$ & $\begin{array}{l}\text { 34. Nummus similis n. }{ }^{\circ} 29 \text {. } \\
\mathrm{R}^{\circ} \text { / }\end{array}$ & 218(d) Rm (244-246) & $\begin{array}{l}\text { A1350 } \\
\text { g 3,40; mm 23; h } 12\end{array}$ \\
\hline$\frac{\text { idem }}{\left(\text { arg. }^{\text {to }}\right)}$ & $\begin{array}{l}\text { 35. Similis antecedenti. } \\
\mathrm{R}^{\circ} \%\end{array}$ & 219 Rm (244-246) & $\begin{array}{l}\text { B5188 } \\
\text { g 4,08; mm 23; h } 12\end{array}$ \\
\hline \multirow[t]{2}{*}{$\begin{array}{l}\text { idem } \\
\text { (arg.to })\end{array}$} & $\begin{array}{l}\text { 36. Alius similis. } \\
\mathrm{R}^{\circ} \%\end{array}$ & $\begin{array}{l}218(d)-219 \mathrm{Rm}(244- \\
246)\end{array}$ & - \\
\hline & $==$ Trajanus Decius ${ }^{47}==$ & & \\
\hline$($ arg.to $)$ & $\begin{array}{l}\text { 37. Imp. Cnaeus Messius Q. Trajanus Decius Caput } \\
\text { Trajani Decii } \\
\text { R\% Victoria Aug. Victoria gradiens, d. lauream sin. } \\
\text { palmam. }\end{array}$ & 7(c) $\mathrm{Rm}(249 ?)$ & $\begin{array}{l}\text { A1367 } \\
\text { g 4,40; mm 23; h } 12\end{array}$ \\
\hline [p.] $\underline{20}$ & $==$ Sequitur Trajanus Decius $==$ & & \\
\hline$\left(\arg .^{\text {to }}\right)$ & $\begin{array}{l}\text { 38. Caput ut supra. } \\
\mathrm{R}^{\circ} \% \text { Dacia. Figura stans, d. baculum cum capite equino. }\end{array}$ & 12(b) Rm (249-251) & $\begin{array}{l}\text { A1359 } \\
\text { g 4,15; mm 22; h } 12\end{array}$ \\
\hline $\begin{array}{l}\text { idem } \\
\left(\text { arg.t }{ }^{\mathrm{to}}\right)\end{array}$ & $\begin{array}{l}\text { 39. Nummus alius similis. } \\
\mathrm{R}^{\circ} /\end{array}$ & c.s. & $\begin{array}{l}\text { A1361 } \\
\text { g } 4,15 ; \mathrm{mm} 22 ; \mathrm{h} 7\end{array}$ \\
\hline $\begin{array}{l}\text { idem } \\
\left(\text { arg. }^{\text {to }}\right)\end{array}$ & $\begin{array}{l}40 \text { Idem similis. } \\
\mathrm{R}^{\circ} /\end{array}$ & c.s. & $\begin{array}{l}\text { A1362 } \\
\text { g 3,20; mm 22; h } 1\end{array}$ \\
\hline $\begin{array}{l}\text { idem } \\
\left(\text { arg. }^{\text {to }}\right)\end{array}$ & $\begin{array}{l}41 \text { Similis. } \\
\mathrm{R}^{\circ} \%\end{array}$ & $\begin{array}{l}\text { 2(b), 12(b) Rm (249- } \\
251)\end{array}$ & - \\
\hline $\begin{array}{l}\text { idem } \\
\left(\text { arg. }{ }^{\text {to }}\right)\end{array}$ & $\begin{array}{l}\text { 42. Caput ut supra. } \\
\mathrm{R} \% \text { Dacia Felix. Figura stans, d. signum militare. }\end{array}$ & 36(a) Rm (250-251?) & $\begin{array}{l}\text { A1358 } \\
\text { g 3,35; mm 22; h } 6\end{array}$ \\
\hline $\begin{array}{l}\text { idem } \\
\left(\text { arg.t }^{\text {to }}\right)\end{array}$ & $\begin{array}{l}\text { 43. Caput ut supra } \\
\mathrm{R}^{\circ} \text {. Pannonia. Duae figurae stantes cum signis } \\
\text { militaribus }\end{array}$ & 21(b) Rm (249-251) & $\begin{array}{l}\text { A1370 } \\
\text { g } 3,85 ; \mathrm{mm} \mathrm{21;} \mathrm{h} 6\end{array}$ \\
\hline
\end{tabular}

$\overline{47}$ Tutte le monete di Traiano Decio attribuite in RIC, IV, III alla zecca di Mediolanum sono ascritte a quella di Roma in base a ELKS 1978. 


\begin{tabular}{|c|c|c|c|}
\hline & & nominale/RIC/zecca/(data) & Collocazione \\
\hline $\begin{array}{l}\text { idem } \\
\left(\text { arg. }^{\text {.t }}\right)\end{array}$ & $\begin{array}{l}\text { 44 Caput uta supra } \\
\mathrm{R}^{\circ} \text {. Postica fere similis superiori. }\end{array}$ & c.s. & $\begin{array}{l}\text { B5240 } \\
\text { g 4,19; mm 22; h } 12\end{array}$ \\
\hline$\frac{\text { idem }}{\left(\text { arg. }{ }^{\text {to }}\right)}$ & $\begin{array}{l}\text { 45. Alius similis. } \\
\mathrm{R}^{\circ} \%\end{array}$ & c.s. & $\begin{array}{l}\text { B5241 } \\
\text { g 4,00; mm 21; h } 6\end{array}$ \\
\hline $\begin{array}{l}\text { idem } \\
\text { (arg.to })\end{array}$ & $\begin{array}{l}\text { 46. Idem Similis superioribus. } \\
\mathrm{R}^{\circ} \%\end{array}$ & c.s. & - \\
\hline $\begin{array}{l}\text { idem } \\
\left(\text { arg.t }^{\text {to }}\right)\end{array}$ & $\begin{array}{l}\text { 47. Caput ut supra } \\
\mathrm{R}^{\circ} \text {. Adventus Aug. Imperator Eques. }\end{array}$ & 11(b) Rm (249-251) & - \\
\hline $\begin{array}{l}\text { idem } \\
\text { (arg.to }\end{array}$ & $\begin{array}{l}\text { 48. Caput ut supra. } \\
\mathrm{R} \% \text {. Annona, ut mihi videtur Aug. Mulier d. spicas } \\
\text { super modium. }\end{array}$ & $?$ & - \\
\hline [p.] $\underline{21}$ & $==$ Sequitur $\underline{\text { Trajanus Decius }}==$ & & \\
\hline $\begin{array}{l}\text { idem } \\
\left(\text { arg.t }^{\text {to }}\right)\end{array}$ & $\begin{array}{l}49 \text { Caput ut supra } \\
\mathrm{R}^{\%} \text {. Genius Illyrici. Genius stans, d. pateram, sin. } \\
\text { cornucopiae. }\end{array}$ & 15(b) Rm (249-251) & $\begin{array}{l}\text { A1364 } \\
\text { g 3,10; mm 21; h } 1\end{array}$ \\
\hline $\begin{array}{l}\text { idem } \\
\left(\text { arg. }^{\text {to }}\right)\end{array}$ & $\begin{array}{l}\text { 50. Videtur similis superiori. } \\
\mathrm{R}^{\circ} \text {. }\end{array}$ & c.s. & - \\
\hline$\left.\frac{\text { idem }}{(\text { arg.to }}{ }^{\text {to }}\right)$ & $\begin{array}{l}\text { 51. Caput ut supra. } \\
\mathrm{R} \% \text { Abundantia Aug. Figura stans manu dextera } \\
\text { sinui admota. }\end{array}$ & 10(b) Rm (249-251) & $\begin{array}{l}\text { A1372 } \\
\text { g 4,65; mm 23; h } 11\end{array}$ \\
\hline $\begin{array}{l}\text { idem } \\
\left(\text { arg. }^{\text {to }}\right)\end{array}$ & $\begin{array}{l}\text { 52. Similis superiori. } \\
\mathrm{R}^{\circ} \%\end{array}$ & c.s. & - \\
\hline $\begin{array}{l}\text { idem } \\
(\text { arg.to })\end{array}$ & $\begin{array}{l}3 \text { Caput ut supra } \\
\mathrm{R}^{\%} \text {. Ubertas Augusta. Figura stans, d. Crumenam, } \\
\text { sin. cornucopiae. }\end{array}$ & $28 \mathrm{Rm}(249-251)$ & $\begin{array}{l}\text { B5249 } \\
\text { g 4,81; mm 22; h } 12\end{array}$ \\
\hline $\begin{array}{l}\text { idem } \\
\left(\text { arg.t }{ }^{\circ}\right)\end{array}$ & $\begin{array}{l}\text { 54. Similis superiori. } \\
\mathrm{R}^{\circ} /\end{array}$ & c.s. & $\begin{array}{l}\text { B5250 } \\
\text { g 3,73; mm 22; h } 12\end{array}$ \\
\hline $\begin{array}{l}\text { idem } \\
\left(\text { arg. }^{\text {.t }}\right)\end{array}$ & $\begin{array}{l}55 \text { Idem Similis. } \\
\mathrm{R}^{\circ} \%\end{array}$ & c.s. & $\begin{array}{l}\text { B5252 } \\
\text { g 4,60; mm 23; h } 6\end{array}$ \\
\hline$\left.\frac{\text { idem }}{(\text { arg.to }}\right)$ & $\begin{array}{l}\text { 56. Caput ut supra } \\
\mathrm{R}^{\circ} / \text { Victoria Aug. Victoria gradiens, d. lauream, sin. } \\
\text { palmam. }\end{array}$ & 29(c) Rm (249-251) & $\begin{array}{l}\text { A1368 } \\
\text { g 4,20; mm 23; h } 6\end{array}$ \\
\hline $\begin{array}{l}\text { idem } \\
(\text { arg.to }\end{array}$ & $\begin{array}{l}57 \text { Similis superiori. } \\
\mathrm{R}^{\circ} /\end{array}$ & c.s. & $\begin{array}{l}\text { B5254 } \\
\text { g 3,14; mm 22; h } 6\end{array}$ \\
\hline $\begin{array}{l}\text { idem } \\
\left(\text { arg. }^{\text {.t }}\right)\end{array}$ & $\begin{array}{l}\text { 58. Idem Similis. } \\
\mathrm{R}^{\circ} \%\end{array}$ & c.s. & $\begin{array}{l}\text { B5256 } \\
\text { g 3,90; mm 22; h } 6\end{array}$ \\
\hline $\begin{array}{l}\text { idem } \\
(\text { arg.to }\end{array}$ & $\begin{array}{l}\text { 59 Similis. } \\
\mathrm{R}^{\circ} /\end{array}$ & c.s. & - \\
\hline [p.] $\underline{22}$ & $==$ Sequitur Trajanus Decius $==$ & & \\
\hline \multirow[t]{2}{*}{$\left(\arg .^{\text {to }}\right)$} & $\begin{array}{l}60 \text { Caput ut supra } \\
\mathrm{R}^{\circ} \% \text { Postica similis antecedenti. }\end{array}$ & c.s. & - \\
\hline & $61==$ Herenia Etruscilla $==$ & & \\
\hline$\left(\arg \cdot{ }^{\mathrm{to}}\right)$ & $\begin{array}{l}\text { 61. Herennia Etruscilla Aug. Caput Etruscillae. } \\
\text { R\% . Pudicitia. Figura sedens, d. ...., sin. hastam. }\end{array}$ & 59(b) Rm (249-251) & $\begin{array}{l}\text { A1390 } \\
\text { g 4,75; mm 23; h } 6\end{array}$ \\
\hline $\begin{array}{l}\text { eadem } \\
\left(\arg ^{\mathrm{t}}{ }^{\circ}\right)\end{array}$ & $\begin{array}{l}\text { 62. Caput ut supra. } \\
\mathrm{R} \% \text {. Fecunditas Aug. Figura stolata stans, d. pateram, } \\
\text { sin. cornucopiae, puerulo pedes assurgente. }\end{array}$ & 55(b) Rm (249-251) & $\begin{array}{l}\text { A1385 } \\
\text { g } 2,95 ; \mathrm{mm} 21 ; \mathrm{h} 7\end{array}$ \\
\hline $\begin{array}{l}\text { eadem } \\
\left(\text { arg. }^{\mathrm{t}}{ }^{\circ}\right)\end{array}$ & $\begin{array}{l}\text { 63. Caput ut supra. } \\
\text { R\% } \% \text { Pudicitia Aug. Mulier stans peplum ori } \\
\text { admovens, sin. hastam. }\end{array}$ & 58(b) Rm (249-251) & $\begin{array}{l}\text { A1387 } \\
\text { g 3,65; mm 22; h } 12\end{array}$ \\
\hline
\end{tabular}




\begin{tabular}{|c|c|c|c|}
\hline & & nominale/RIC/zecca/(data) & Collocazione \\
\hline$\frac{\text { eadem }}{\left(\text { arg. }{ }^{\mathrm{t}}\right)}$ & $\begin{array}{l}\text { 64. Nummus Similis superiori. } \\
\mathrm{R}^{\circ} \%\end{array}$ & c.s. & $\begin{array}{l}\text { A1395 } \\
\text { g 4,35; mm 21; h } 2\end{array}$ \\
\hline $\begin{array}{l}\text { eadem } \\
(\text { arg.to }\end{array}$ & $\begin{array}{l}\text { 65. Similis n. }{ }^{\circ} 61 . \\
\mathrm{R}^{\circ} \%\end{array}$ & 59(b) Rm (249-251) & $\begin{array}{l}\text { A1391 } \\
\text { g 3,80; mm 20; h } 12\end{array}$ \\
\hline$\frac{\text { eadem }}{\left(\text { arg. }{ }^{\mathrm{o}}\right)}$ & $\begin{array}{l}66 \text { Idem Similis n. }{ }^{\circ} 61 . \\
\mathrm{R}^{\circ} \%\end{array}$ & c.s. & $\begin{array}{l}\text { A1392 } \\
\text { g 4,25; mm 22; h } 6\end{array}$ \\
\hline $\begin{array}{l}\text { eadem } \\
(\text { arg.to }\end{array}$ & $\begin{array}{l}\text { 67. Similis n. }{ }^{\circ} 61 . \\
\mathrm{R}^{\circ} \%\end{array}$ & c.s. & $\begin{array}{l}\text { A1393 } \\
\text { g 4,40; mm 22; h } 6\end{array}$ \\
\hline$\frac{\text { eadem }}{\left(\text { arg. }{ }^{\text {to }}\right)}$ & $\begin{array}{l}\text { 68 Alius simils. } \\
\mathrm{R}^{\circ} \%\end{array}$ & c.s. & $\begin{array}{l}\text { A1394 } \\
\text { g 4,70; mm 22; h } 12\end{array}$ \\
\hline$\frac{\text { eadem }}{\left(\text { arg. }{ }^{\circ}\right)}$ & $\begin{array}{l}69 \text { Idem Similis } \\
\mathrm{R}^{\circ} \%\end{array}$ & c.s. & $\begin{array}{l}\text { B5312 } \\
\text { g 3,87; mm 22; h } 6\end{array}$ \\
\hline$\frac{\text { eadem }}{\left(\text { arg. }{ }^{\mathrm{t}}\right)}$ & $\begin{array}{l}70 \text { Similis. } \\
\mathrm{R}^{\circ} \%\end{array}$ & c.s. & ( \\
\hline \multicolumn{4}{|c|}{$\begin{array}{l}\text { Cassella II } \\
\text { Cartella D }\end{array}$} \\
\hline [p.] $\underline{23}$ & $==$ Trebonianus Gallus ${ }^{48}==$ & & \\
\hline$\left(\arg { }^{\text {to }}\right)$ & $\begin{array}{l}\text { 1. Imp. Caesar Caius Vibius Trebonianus Gallus Aug. } \\
\text { Caput ejusdem coromatum. } \\
\mathrm{R}^{\circ} / \text { Victoria Augusta stans, } \text { d. coronam, sin. } \\
\text { bacillum dimissum. }\end{array}$ & $\begin{array}{l}48 \mathrm{Rm}, 93-95 \text { Ant } \\
(251-253)\end{array}$ & - \\
\hline$\frac{\text { idem }}{(\text { arg.to }}$ & $\begin{array}{l}2 \text { Caput ut supra. } \\
\mathrm{R} \% \text { Saeculum novum. Templum sex columnarum, in } \\
\text { cujus medio fortasse figura sedens. }\end{array}$ & 90 Ant (251-253) & - \\
\hline$\frac{\text { idem }}{\left(\text { arg. }{ }^{\text {to }}\right)}$ & $\begin{array}{l}\text { 3. Caput ut supra. } \\
\mathrm{R}^{\circ} \% \text { Annona Aug. Figura stans, d. hastam, sin. palmam. }\end{array}$ & 31 Rm (251-253) & $\begin{array}{l}\text { A1448 } \\
\text { g 3,39; mm 21; h } 6\end{array}$ \\
\hline $\begin{array}{l}\text { idem } \\
\left(\text { arg. }^{\text {.o }}\right)\end{array}$ & $\begin{array}{l}4 \text { Caput ut supra } \\
\text { R/ Postica similis superiori. }\end{array}$ & c.s. & 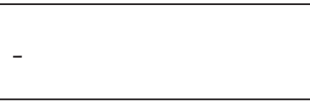 \\
\hline$\frac{\text { idem }}{\left(\text { arg.t }{ }^{\mathrm{to}}\right)}$ & $\begin{array}{l}\text { 5. Caput ut supra. } \\
\mathrm{R}^{\circ} / \text { Aequitas Augustorum. Figura stans, d. bilancem, } \\
\text { sin. cornucopiae. }\end{array}$ & $\begin{array}{l}\text { Probabilmente con- } \\
\text { fuso con Volusiano } \\
\text { RIC } 166 \mathrm{Rm}(251- \\
253)\end{array}$ & $\begin{array}{l}\text { A1496 } \\
\text { g 3,75; mm 21,5; h } 1\end{array}$ \\
\hline $\begin{array}{l}\text { idem } \\
(\text { arg.to })\end{array}$ & $\begin{array}{l}\text { 6. Caput ut supra. } \\
\mathrm{R}^{\circ} \text { / Felicitas Publica. d. caduceum, sin. baculum vel } \\
\text { hastam transversam. }\end{array}$ & 34A Rm (252-253) & - \\
\hline $\begin{array}{l}\text { idem } \\
(\text { arg.to }\end{array}$ & $\begin{array}{l}\text { 7. Alius similis superiori. } \\
\mathrm{R}^{\circ} \text { Apollini Salutari. Apollo d. ramum lauri sin. } \\
\text { Cytharam. }\end{array}$ & 32 Rm (251-253) & - \\
\hline$\frac{\text { idem }}{\left(\text { arg.t }{ }^{\text {to }}\right)}$ & $\begin{array}{l}8 \text { Caput ut supra. } \\
\mathrm{R}^{\circ} / \text {. Similis postica } \text { n. }^{\circ} 6 .\end{array}$ & $\begin{array}{l}\text { Probabilmenter } \text { Feli- } \\
\text { citas Publica RIC } 34 \mathrm{~A} \\
\mathrm{Rm}(252-253) \text { v. supra }\end{array}$ & - \\
\hline $\begin{array}{l}\text { idem } \\
(\text { arg.t. })\end{array}$ & $\begin{array}{l}9 \text { Caput ut supra } \\
\mathrm{R} \% \text { Libertas publica. Figura stans, d. pileum sin. } \\
\text { hastam transversam. }\end{array}$ & 70 Rm (251-253) & $\begin{array}{l}\text { A1419 } \\
\text { g 2,95; mm 20,5; h } 6\end{array}$ \\
\hline [p.] 24. & $==$ Sequitur Trebonianus Gallus $==$ & & \\
\hline$\left(\arg \cdot{ }^{\text {to }}\right)$ & $\begin{array}{l}\text { 10. Nummis similis n. }{ }^{\circ} 8 \text {. [probabilmente n. 9, n.d.r.] } \\
\mathrm{R}^{\circ} \%\end{array}$ & c.s. & $\begin{array}{l}\text { A1421 } \\
\text { g 4,23; mm 22; h } 6\end{array}$ \\
\hline
\end{tabular}

\footnotetext{
$\overline{{ }^{48}}$ Tutte le monete di Treboniano Gallo attribuite in RIC, IV, III alla zecca di Mediolanum sono ascritte a quella di
} Roma in base a ELKS 1978. 


\begin{tabular}{|c|c|c|c|}
\hline & & nominale/RIC/zecca/(data) & Collocazione \\
\hline $\begin{array}{l}\text { idem } \\
\left(\text { arg. }{ }^{+0}\right)\end{array}$ & $\begin{array}{l}\text { 11. Similis superiori } \\
\mathrm{R}^{\circ} /\end{array}$ & c.s. & $\begin{array}{l}\text { A1435 } \\
\text { g 4,27; mm 22; h } 1\end{array}$ \\
\hline $\begin{array}{l}\text { idem } \\
(\text { arg.to) }\end{array}$ & $\begin{array}{l}\text { 12. Idem similis. } \\
\mathrm{R} \%\end{array}$ & c.s. & $\begin{array}{l}\text { A1444 } \\
\text { g 3,33; mm 22; h } 6\end{array}$ \\
\hline $\begin{array}{l}\text { idem } \\
(\text { arg.t. }\end{array}$ & $\begin{array}{l}\text { 13. Idem similis. } \\
\quad \text { Caput ut supra. } \\
\mathrm{R} \% \text { Libertas Augg. Figura muliebris, d. pileum, sin. } \\
\text { columnae innixa. }\end{array}$ & $39 \mathrm{Rm}(251-253)$ & $\begin{array}{l}\text { A1416 } \\
\text { g 3,28; mm 23; h } 6\end{array}$ \\
\hline $\begin{array}{l}\text { idem } \\
\left(\text { arg. }^{\text {to }}\right)\end{array}$ & $\begin{array}{l}14 \text { Caput ut supra. } \\
\mathrm{R}^{\circ} \text {. Postica similis n. } 11 \text { et aliis [probabilmente } \\
\text { come n. } 13, \text { n.d.r.]. }\end{array}$ & 37 Rm (251-253) & $\begin{array}{l}\text { A1449 } \\
\text { g } 4,10 ; \mathrm{mm} \mathrm{22;} \mathrm{h} 1\end{array}$ \\
\hline$\frac{\text { idem }}{\left(\text { arg.t }{ }^{\text {to }}\right)}$ & $\begin{array}{l}\text { 15. Caput ut supra } \\
\mathrm{R}^{\circ} \% \text { Pax Aeterna. Figura stolata, d. ramum, sin. ha- } \\
\text { stam transversam. }\end{array}$ & $71 \mathrm{Rm}(251-253)$ & $\begin{array}{l}\text { A1446 } \\
\text { g 3,11; mm 23; h } 6\end{array}$ \\
\hline $\begin{array}{l}\text { idem } \\
\left(\text { arg.t }{ }^{\circ}\right)\end{array}$ & $\begin{array}{l}16 \text { Similis superiori } \\
\mathrm{R} \%\end{array}$ & c.s. & $\begin{array}{l}\text { A1447 } \\
\text { g } 3,94 ; \mathrm{mm} 22 ; \mathrm{h} 12\end{array}$ \\
\hline $\begin{array}{l}\text { idem } \\
(\text { arg.to }\end{array}$ & $\begin{array}{l}\text { 17. Similis n. }{ }^{\circ} 11 \text { [probabilmente come n. } 13, \text { n.d.r.]. } \\
\mathrm{R}^{\circ} \%\end{array}$ & 39 Rm (251-253) & $\begin{array}{l}\text { A1436 } \\
\text { g 3,39; mm 20; h } 8\end{array}$ \\
\hline $\begin{array}{l}\text { idem } \\
\left(\text { arg. }^{\text {to }}\right)\end{array}$ & $\begin{array}{l}18 \text { Similis n. }{ }^{\circ} 15 \text {. } \\
\mathrm{R}^{\circ} \%\end{array}$ & 71 Rm (251-253) & $\begin{array}{l}\text { A1452 } \\
\text { g 3,13; mm 21; h } 6\end{array}$ \\
\hline $\begin{array}{l}\text { idem } \\
\left(\text { arg.to }^{\text {to }}\right)\end{array}$ & $\begin{array}{l}\text { 19. Caput ut supra. } \\
\mathrm{R} \% \text { Pietas Aug. Figura stans ad aram, et utramque } \\
\text { manum attollens. }\end{array}$ & 72 Rm (251-253) & $\begin{array}{l}\text { A1427 } \\
\text { g 2,99; mm 22; h } 12\end{array}$ \\
\hline [p.] $\underline{25}$ & $==$ Sequitur Trebonians Gallus $==$ & & \\
\hline$\left(\arg .^{\text {to }}\right)$ & $\begin{array}{l}\text { 20. Nummus similis antecedenti. } \\
\mathrm{R}^{\circ} /\end{array}$ & $41 \mathrm{Rm}(251-253)$ & $\begin{array}{l}\text { A1423 } \\
\text { g 4,24; mm 20; h } 5\end{array}$ \\
\hline $\begin{array}{l}\text { idem } \\
(\text { arg.to })\end{array}$ & $\begin{array}{l}21 \text { Alius similis. } \\
\mathrm{R}^{\circ} \%\end{array}$ & c.s. & $\begin{array}{l}\text { A1424 } \\
\text { g 3,65; mm 21; h } 6\end{array}$ \\
\hline$\left.\frac{\text { idem }}{(\text { arg.t }}{ }^{\text {to }}\right)$ & $\begin{array}{l}\text { 22. Idem alius. } \\
\mathrm{R}^{\circ} \text {. }\end{array}$ & $42 \mathrm{Rm}(251-253)$ & $\begin{array}{l}\text { A1453 } \\
\text { g 3,71; mm 22; h } 7\end{array}$ \\
\hline$\frac{\text { idem }}{\left(\text { arg. }^{\text {to }}\right)}$ & $\begin{array}{l}23 \text { Idem Similis } \\
\mathrm{R}^{\circ} \%\end{array}$ & c.s. & - \\
\hline $\begin{array}{l}\text { idem } \\
\left(\text { arg.t }{ }^{\circ}\right)\end{array}$ & $\begin{array}{l}24 \text { Similis } \\
\left.\mathrm{R}^{\circ} \text {. [testo cancellato illeggibile, } n . d . r .\right]\end{array}$ & c.s. & - \\
\hline$\frac{\text { idem }}{(\text { arg.to })}$ & $\begin{array}{l}\text { 25. Caput ut supra. } \\
\mathrm{R} \% \text { Salus Augusta. Figura stans d. pascit serpentem } \\
\text { in patera, sin. hastam. }\end{array}$ & $47 \mathrm{Rm}(251-253)$ & $\begin{array}{l}\text { A1443 } \\
\text { g 3,03; mm 22; h } 1\end{array}$ \\
\hline $\begin{array}{l}\text { idem } \\
\left(\text { arg.t }^{\text {to }}\right)\end{array}$ & $\begin{array}{l}26 \text { Caput ut supra. } \\
\mathrm{R} \% \text { Juno Martialis. Figura sedens d. ramum, sin. } \\
\text { hastam puram. }\end{array}$ & 35 Rm (251-253) & $\begin{array}{l}\text { A1433 } \\
\text { g 4,26; mm 22; h } 12\end{array}$ \\
\hline $\begin{array}{l}\text { idem } \\
\left(\text { arg. }^{\text {to }}\right)\end{array}$ & $\begin{array}{l}27 \text { Caput ut supra. } \\
\mathrm{R} \% \text { Victoria Augustorum. Typus Victoriae stantis. }\end{array}$ & 48a Rm (251-253) & $\begin{array}{l}\text { A1428 } \\
\text { g 3,74; mm 22; h } 6\end{array}$ \\
\hline$\frac{\text { idem }}{(\text { arg.t }}$ & $\begin{array}{l}\text { 28. Nummus similis superiori. } \\
\mathrm{R} \%\end{array}$ & c.s. & $\begin{array}{l}\text { A1429 } \\
\text { g 3,94; mm 21; h } 6\end{array}$ \\
\hline$\frac{\text { idem }}{\text { (arg.to })}$ & $\begin{array}{l}29 \text { Idem Similis } \\
\mathrm{R}^{\circ} \%\end{array}$ & c.s. & $\begin{array}{l}\text { A1431 } \\
\text { g 3,59; mm 20; h } 12\end{array}$ \\
\hline$\frac{\text { idem }}{\text { (arg.t }}$ & $\begin{array}{l}30 \text { Similis } \\
\mathrm{R}^{\circ} \%\end{array}$ & $\begin{array}{l}48 \text { Rm, 93-95 Ant } \\
(251-253)\end{array}$ & - \\
\hline
\end{tabular}




\begin{tabular}{|c|c|c|c|}
\hline & & nominale/RIC/zecca/(data) & Collocazione \\
\hline [p.] $\underline{26}$ & $==$ Sequitur Trebonianus Gallus $==$ & & \\
\hline$\left(\arg .^{\text {to }}\right)$ & $\begin{array}{l}\text { 31. Caput ut supra } \\
\mathrm{R}^{\circ} \text {. Virtus Augg. Figura militaris, d. hastam, sin. } \\
\text { scuto innixa. }\end{array}$ & $\begin{array}{l}\text { 57-58, } 76 \mathrm{Rm}, 96 \text { Ant } \\
(251-253)\end{array}$ & - \\
\hline$\frac{\text { idem }}{\left(\text { arg. }^{\text {to }}\right)}$ & $\begin{array}{l}\text { 32. Similis n. }{ }^{\circ} 20 \text { [probabilmente } 26^{49} \text {, n.d.r.]. } \\
\mathrm{R}^{\circ} \text {. }\end{array}$ & 35 Rm (251-253) & $\begin{array}{l}\text { B5371 } \\
\text { g } 3,47 ; \mathrm{mm} \mathrm{22;} \mathrm{h} 6\end{array}$ \\
\hline$\frac{\text { idem }}{\left(\text { arg. }{ }^{\text {to }}\right)}$ & $\begin{array}{l}\text { 33. Alius similis superiori. } \\
\mathrm{R}^{\circ} \text {. }\end{array}$ & c.s. & - \\
\hline $\begin{array}{l}\text { idem } \\
(\text { (arg.to })\end{array}$ & $\begin{array}{l}34 \text { Idem Similis. } \\
\mathrm{R}^{\circ} /\end{array}$ & c.s. & - \\
\hline $\begin{array}{l}\text { idem } \\
\left(\text { arg. }^{\text {to }}\right)\end{array}$ & $\begin{array}{l}35 \text { Similis. } \\
\mathrm{R} \%\end{array}$ & c.s. & - \\
\hline$\frac{\text { idem }}{\left(\text { arg. }{ }^{\circ} \text { ) }\right.}$ & $\begin{array}{l}36 \text { Idem Similis } \\
\mathrm{R}^{\circ} \%\end{array}$ & c.s. & - \\
\hline \multirow[t]{2}{*}{$\frac{\text { idem }}{\left(\text { arg. }^{\circ}\right)}$} & $\begin{array}{l}\text { 37. Caput, ut supra } \\
\mathrm{R} \% \text {....ritas. Figura stans, d. ut videtur Globum sin } \\
\text { cornucopiae. }\end{array}$ & 30 (?) Rm (251-253) & - \\
\hline & $==C$. Vibius Volusianus $==$ & & \\
\hline$($ arg.to $)$ & $\begin{array}{l}38 \text { Imp. Caes. C. Vibio Volusiano Aug. [testo cancellato } \\
\text { illeggibile, n.d.r.] Caput Volusiani laureatum } \\
\text { R\% Concordia Augg. Figura stans, d. pateram, sin. } \\
\text { cornucopiae. }\end{array}$ & 167 Rm (251-253) & $\begin{array}{l}\text { A1461 } \\
\text { g 3,55; mm 21; h } 8\end{array}$ \\
\hline $\begin{array}{l}\text { idem } \\
\left(\text { arg. }^{\mathrm{to}}\right)\end{array}$ & $\begin{array}{l}39 \text { Similis Superiori. } \\
\mathrm{R}^{\circ} /\end{array}$ & c.s. & $\begin{array}{l}\text { A1484 } \\
\text { g 3,36; mm21,5; h } 1\end{array}$ \\
\hline [p.] 27 & $==$ Sequitur Volusianus ${ }^{50}==$ & & \\
\hline$\left(\right.$ arg.t ${ }^{\text {to }}$ & $\begin{array}{l}40 \text { Similis antecedenti. } \\
\mathrm{R} \%\end{array}$ & c.s. & $\begin{array}{l}\text { A1487 } \\
\text { g 3,37; mm 22; h } 11\end{array}$ \\
\hline$\frac{\text { idem }}{\left(\text { arg. }^{\text {to }}\right)}$ & $\begin{array}{l}41 \text { Idem Similis. } \\
\mathrm{R}^{\circ}\end{array}$ & c.s. & $\begin{array}{l}\text { A1498 } \\
\text { g 3,61; mm 21; h } 12\end{array}$ \\
\hline$\frac{\text { idem }}{(\text { arg.to }}$ & $\begin{array}{l}42 \text { Caput ut supra. } \\
\mathrm{R}^{\circ} / \text { Felicitas Publica. Figura stans, d. caduceum } \\
\text { oblongum, sin. cornucopiae. }\end{array}$ & 188A Rm (251-253) & $\begin{array}{l}\text { B5363 } \\
\text { g 3,37; mm 22; h } 6\end{array}$ \\
\hline$\frac{\text { idem }}{\left(\arg .^{\circ}\right)}$ & $\begin{array}{l}\text { 43. Similis superiori. } \\
\mathrm{R}^{\circ} /\end{array}$ & 205 Rm (251-253) & $\begin{array}{l}\text { A1483 } \\
\text { g 3,92; mm 23; h } 12\end{array}$ \\
\hline$\frac{\text { idem }}{\left(\text { arg. }^{\text {to }}\right)}$ & $\begin{array}{l}44 \text { Alius similis. } \\
\mathrm{R}^{\circ} \%\end{array}$ & c.s. & $\begin{array}{l}\text { A1493 } \\
\text { g } 4,17 ; \mathrm{mm} 22 ; \mathrm{h} 12\end{array}$ \\
\hline $\begin{array}{l}\text { idem } \\
\left(\arg ^{\text {to }}\right)\end{array}$ & $\begin{array}{l}45 \text { Idem Similis } \\
\mathrm{R}^{\circ}\end{array}$ & c.s. & $\begin{array}{l}\text { A1497 } \\
\text { g 2,72; mm 22; h } 1\end{array}$ \\
\hline$\frac{\text { idem }}{\left(\text { arg. }^{\mathrm{t}}{ }^{\circ}\right)}$ & $\begin{array}{l}46 \text { Alius similis. } \\
\mathrm{R}^{\circ} \%\end{array}$ & c.s. & $\begin{array}{l}\text { A1502 } \\
\text { g 3,63; mm 24; h } 5\end{array}$ \\
\hline
\end{tabular}

\footnotetext{
${ }^{49}$ Del tipo Iuno Martialis esistono due esemplari, l'uno presso il Museo Archeologico Nazionale di Venezia (v. supra, n. 26), l'altro presso il Museo Correr di Venezia, entrambi accomunati dalla medesima patina e dunque certamente pertinenti al ripostiglio. Nel manoscritto con quella tipologia è descritto solo il numero 26 per Treboniano Gallo. Le uniche soluzioni che sembrano percorribili per risolvere questa incongruenza riguardano, a nostro giudizio, un errore nel conteggio delle monete, oppure un errore di indicazione numerica nella descrizione della moneta $\mathrm{n}$. 32: qui si riporta la formula "Similis n. '20". Considerato che esemplari silimi al n. 20 sono già elencati sopra (nn. 19-24), ci sembra probabile che qui si volesse alludere ad un altro tipo e che per errore si sia indicato 20 anziché 26.

${ }^{50}$ Tutte le monete di Volusiano attribuite in RIC, IV, III alla zecca di Mediolanum sono ascritte a quella di Roma in base a ELKS 1978.
} 


\begin{tabular}{|c|c|c|c|}
\hline & & nominale/RIC/zecca/(data) & Collocazione \\
\hline $\begin{array}{l}\text { idem } \\
\left(\text { arg. }^{\text {.t }}\right)\end{array}$ & $\begin{array}{l}47 \text { Idem Similis. } \\
\mathrm{R}^{\circ} \%\end{array}$ & c.s. & $\begin{array}{l}\text { A1503 } \\
\text { g 3,60; mm 24; h } 12\end{array}$ \\
\hline$\frac{\text { idem }}{\left(\text { arg.t }{ }^{\mathrm{t}}\right)}$ & $\begin{array}{l}48 \text { Caput ut supra. } \\
\text { R\% . Libertas Aug. Figura stans, d. pileum sin. } \\
\text { columnae innixa }\end{array}$ & $190 \mathrm{Rm}(251-253)$ & - \\
\hline $\begin{array}{l}\text { idem } \\
\left(\text { arg. }^{\text {to }}\right)\end{array}$ & $\begin{array}{l}49 \text { Caput ut supra. } \\
\mathrm{R} \% \text { Pax Aug. Mulier stans d. ramum sin hastam } \\
\text { transversam. In area stella } \\
\end{array}$ & $180 \mathrm{Rm}(251-253)$ & $\begin{array}{l}\text { A1462 } \\
\text { g } 3,77 ; \mathrm{mm} 21 ; \mathrm{h} 12\end{array}$ \\
\hline [p.] $\underline{28}$ & $==$ Sequitur C. Vib. Volusianus $==$ & & \\
\hline$\frac{\text { idem }}{\left(\text { arg.t }{ }^{\text {to }}\right)}$ & $\begin{array}{l}\text { 50. Similis antecedenti. } \\
\mathrm{R}^{\circ} \text {. }\end{array}$ & c.s. & $\begin{array}{l}\text { A1466 } \\
\text { g 3,97; mm 21; h } 12\end{array}$ \\
\hline$\frac{\text { idem }}{\left(\text { arg. }^{\text {to }}\right)}$ & $\begin{array}{l}\text { 51. Idem similis } \\
\mathrm{R}^{\circ} \text {. }\end{array}$ & c.s. & $\begin{array}{l}\text { A1468 } \\
\text { g 3,72; mm 22; h } 12\end{array}$ \\
\hline$\frac{\text { idem }}{\left.\text { (arg. }{ }^{\text {to }}\right)}$ & $\begin{array}{l}\text { 52. Idem similis. } \\
\mathrm{R} \%\end{array}$ & c.s. & $\begin{array}{l}\text { A1478 } \\
\text { g 3,38; mm 21; h } 12\end{array}$ \\
\hline$\frac{\text { idem }}{\left(\text { arg.t }{ }^{\mathrm{t}}\right)}$ & $\begin{array}{l}\text { 53. Similis. } \\
\mathrm{R}^{\circ} \text {. }\end{array}$ & c.s. & $\begin{array}{l}\text { A1490 } \\
\text { g 3,01; mm 23; h } 5\end{array}$ \\
\hline$\frac{\text { idem }}{(\text { arg.to }}$ & $\begin{array}{l}\text { 54. Similis } \\
\mathrm{R} \%\end{array}$ & 179 Rm (251-253) & $\begin{array}{l}\text { A1463 } \\
\text { g 3,90; mm 20; h } 7\end{array}$ \\
\hline$\frac{\text { idem }}{\left(\text { arg. }{ }^{\circ}\right)}$ & $\begin{array}{l}\text { 55. Similis. } \\
\mathrm{R}^{\circ} \%\end{array}$ & c.s. & $\begin{array}{l}\text { A1465 } \\
\text { g 4,36; mm 20; h } 12\end{array}$ \\
\hline $\begin{array}{l}\text { idem } \\
\left(\text { arg.t }{ }^{\text {to }}\right)\end{array}$ & $\begin{array}{l}56 \text { Similis. } \\
\mathrm{R} \%\end{array}$ & c.s. & $\begin{array}{l}\text { A1467 } \\
\text { g 3,25; mm 20; h } 12\end{array}$ \\
\hline$\frac{\text { idem }}{(\text { arg. }}$ & $\begin{array}{l}\text { 57. Similis } \\
\mathrm{R}^{\circ} \text { / }\end{array}$ & $\begin{array}{l}179-181 \quad \mathrm{Rm} \quad(251- \\
253)\end{array}$ & 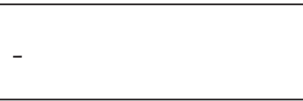 \\
\hline $\begin{array}{l}\text { idem } \\
(\text { arg.to })\end{array}$ & $\begin{array}{l}\text { 58. Caput ut supra } \\
\mathrm{R}^{\circ} \text {. Pietas Augusta. Mulier sagrificans }[\text { sic } ! \text { ] } \underline{\text { ad aram. }} .\end{array}$ & 182 Rm (251-253) & $\begin{array}{l}\text { A1480 } \\
\text { g 3,60; mm 22; h } 6\end{array}$ \\
\hline $\begin{array}{l}\text { idem } \\
\left(\text { arg.t }^{\mathrm{t}}\right)\end{array}$ & $\begin{array}{l}59 \text { Caput ut supra. } \\
\text { R\%. Pont. Max. Tr. P. IIII Cos. Figura sacrificans ad } \\
\text { aram, sin. [testo cancellato illeggibile, n.d.r.] bacu- } \\
\underline{\text { lum [testo cancellato illeggibile, n.d.r.] } \underline{\text { demissum }}}\end{array}$ & $141 \mathrm{Rm}(253)$ & $\begin{array}{l}\text { B5425 } \\
\text { g 3,65; mm 20; h } 6\end{array}$ \\
\hline [p.] $\underline{29}$ & $==$ Sequitur $C$. Vibius Volusianus $==$ & & \\
\hline $\begin{array}{l}\text { idem } \\
\text { (arg.to })\end{array}$ & $\begin{array}{l}60 \text { Caput ut supra. } \\
\mathrm{R} / \% \text {. M. Tr. P. IIII. [testo cancellato illeggibile, } \\
\text { n.d.r.] Cos. I. Figura stans ad aram cum patera et ba- } \\
\text { cillo transverso. }\end{array}$ & $141 \mathrm{Rm}(253)$ & $\begin{array}{l}\text { B5427 } \\
\text { g 3,96; mm 21; h } 6\end{array}$ \\
\hline $\begin{array}{l}\text { idem } \\
(\text { arg.to }\end{array}$ & $\begin{array}{l}\text { 61. Alius similis. } \\
\mathrm{R}^{\circ} \%\end{array}$ & c.s. & - \\
\hline$\frac{\text { idem }}{\left(\text { arg.t }{ }^{\text {to }}\right)}$ & $\begin{array}{l}\text { 62. Caput ut supra. } \\
\text { R\%. P. M. Tr. P. IIII. Cos. II. Figura velata stans d. ra- } \\
\underline{\left.\text { mum, sin. bacillum transversum. (idem similis } \mathrm{n} .^{\circ} 60\right)}\end{array}$ & $140 \mathrm{Rm}(253)$ & $\begin{array}{l}\text { A1470 } \\
\text { g 3,12; mm 20; h } 6\end{array}$ \\
\hline$\frac{\text { idem }}{\left(\text { arg. }{ }^{\text {to }}\right)}$ & $\begin{array}{l}\text { 63. Similis n. }{ }^{\circ} 60 \text {. Figura ramum pro patera tenens } \\
\mathrm{R}^{\circ} \%\end{array}$ & c.s. & $\begin{array}{l}\text { A1481 } \\
\text { g 3,47; mm 20; h } 7\end{array}$ \\
\hline$\frac{\text { idem }}{\left(\text { arg. }^{\text {to }}\right)}$ & $\begin{array}{l}\text { 64 Similis n. } 62 \text {. } \\
\mathrm{R}^{\circ} \%\end{array}$ & c.s. & $\begin{array}{l}\text { A1492 } \\
\text { g 3,75; mm 21; h } 6\end{array}$ \\
\hline$\frac{\text { idem }}{\left(\text { arg. }{ }^{\text {to }}\right)}$ & $\begin{array}{l}\text { 65. Idem Similis superiori. } \\
\mathrm{R}^{\circ} \%\end{array}$ & c.s. & $\begin{array}{l}\text { A1495 } \\
\text { g 3,30; mm 21; h } 6\end{array}$ \\
\hline$\frac{\text { idem }}{(\text { arg.t }}$ & $\begin{array}{l}\text { 66 Similis. } \\
\mathrm{R}^{\circ} \%\end{array}$ & c.s. & $\begin{array}{l}\text { B5423 } \\
\text { g 3,27; mm 22; h } 11\end{array}$ \\
\hline
\end{tabular}




\begin{tabular}{|c|c|c|c|}
\hline & & nominale/RIC/zecca/(data) & Collocazione \\
\hline$\frac{\text { idem }}{\left(\text { arg. }{ }^{\text {to }}\right)}$ & $\begin{array}{l}67 \text { Similis. } \\
\mathrm{R} \%\end{array}$ & c.s. & - \\
\hline$\frac{\text { idem }}{\left(\text { arg. }{ }^{\text {to }}\right)}$ & $\begin{array}{l}\text { 68. Caput ut supra. } \\
\mathrm{R}^{\circ} / \text { Figura Salus Augg. Figura stans cum serpente. }\end{array}$ & 184 Rm (251-253) & $\begin{array}{l}\text { B5428 } \\
\text { g 3,93; mm 20; h } 12\end{array}$ \\
\hline$\frac{\text { idem }}{\left(\text { arg. }{ }^{\circ}\right)}$ & $\begin{array}{l}69 \text { Alius similis } \\
\mathrm{R}^{\circ} \%\end{array}$ & c.s. & $\begin{array}{l}\text { B5429 } \\
\text { g } 4,72 ; \mathrm{mm} 22 ; \mathrm{h} 6\end{array}$ \\
\hline [p.] 30. & $==$ Sequitur Volusianus $==$ & & \\
\hline $\begin{array}{l}\text { idem } \\
\left(\text { arg.t }^{\text {to }}\right)\end{array}$ & $\begin{array}{l}\text { 70. Caput ut supra } \\
\mathrm{R}^{\circ} / \text { Securitas perpetua. Figura stans, d. hastam sin. } \\
\text { columnae innixa. }\end{array}$ & $136 \mathrm{Rm}(251)$ & - \\
\hline$\frac{\text { idem }}{\left(\text { arg.t }{ }^{\text {to }}\right)}$ & $\begin{array}{l}71 \text { Caput ut supra. } \\
\mathrm{R}^{\circ} \% \text { Virtus Aug. Figura stans d. hastam sin. scuto in- } \\
\text { nixa. }\end{array}$ & 206 Rm (251-253) & $\begin{array}{l}\text { A } 1472 \\
\text { g } 3,78 ; \mathrm{mm} 23 ; \mathrm{h} 6\end{array}$ \\
\hline $\begin{array}{l}\text { idem } \\
\left(\text { arg. }^{\text {to }}\right)\end{array}$ & $\begin{array}{l}72 \text { Similis superiori. } \\
\mathrm{R}^{\circ} /\end{array}$ & c.s. & $\begin{array}{l}\text { A1473 } \\
\text { g 3,90; mm 21; h } 12\end{array}$ \\
\hline$\frac{\text { idem }}{\left(\text { arg.t }{ }^{\text {to }}\right)}$ & $\begin{array}{l}73 \text { Idem Similis. } \\
\mathrm{R}^{\circ} /\end{array}$ & c.s. & $\begin{array}{l}\text { A1474 } \\
\text { g 5,06; mm 22; h } 6\end{array}$ \\
\hline$\frac{\text { idem }}{\left(\text { arg. }{ }^{\circ}\right)}$ & $\begin{array}{l}\text { 74. Similis } \\
\mathrm{R}^{\circ} /\end{array}$ & c.s. & $\begin{array}{l}\text { A1475 } \\
\text { g } 4,61 ; \mathrm{mm} 21 ; \mathrm{h} 12\end{array}$ \\
\hline$\frac{\text { idem }}{\text { (arg.to })}$ & $\begin{array}{l}75 \text { Similis } \\
\mathrm{R}^{\circ} /\end{array}$ & c.s. & - \\
\hline$\frac{\text { idem }}{\left(\text { arg. } .^{\text {to }}\right)}$ & $\begin{array}{l}76 \text { Similis } \\
\mathrm{R}^{\circ} /\end{array}$ & c.s. & - \\
\hline$\frac{\text { idem }}{\left(\text { arg.t }{ }^{\mathrm{to}}\right)}$ & $\begin{array}{l}77 \text { Caput ut supra. } \\
\mathrm{R}^{\circ} \text { / Virtus Augg. Figura stans dextera scuto innixa, } \\
\text { sin. hastam }\end{array}$ & 186 Rm (251-253) & $\begin{array}{l}\text { B5432 } \\
\text { g 3,76; mm 22; h } 6\end{array}$ \\
\hline \multicolumn{4}{|c|}{$\begin{array}{l}\text { Cassella II } \\
\text { Cartella E }\end{array}$} \\
\hline [p.] $\underline{31}$ & $==$ Sequitur Volusianus $==$ & & \\
\hline$\left(\arg .^{\text {to }}\right)$ & $\begin{array}{l}\text { 78. Caput ut in antecedenti. } \\
\mathrm{R} \% \text { et Postica similis. }\end{array}$ & 187 Rm (251-253) & $\begin{array}{l}\text { A1477 } \\
\text { g 3,85; mm 21; h } 6\end{array}$ \\
\hline$\frac{\text { idem }}{\left(\text { arg.t }{ }^{\mathrm{t}}\right)}$ & $\begin{array}{l}79 \text { Caput ut supra } \\
\mathrm{R} \% \text { Virtus Aug. Figura stans, d. victoriola, sin. scuto } \\
\text { innixa. }\end{array}$ & $\begin{array}{l}\text { Probilmente confuso } \\
\text { con Valeriano MIR, } \\
\text { 36, n. } 811 \mathrm{Vm}(253- \\
\text { 256) v. infra }\end{array}$ & - \\
\hline$\frac{\text { idem }}{\left(\text { arg.t }{ }^{\mathrm{t}}\right)}$ & $\begin{array}{l}80 \text { Caput ut supra } \\
\mathrm{R}^{\circ} / \text { Concordia Aug. Mulier sedens d. pateram, sin. } \\
\text { cornucopiae }\end{array}$ & 168 Rm (251-253) & $\begin{array}{l}\text { A1499 } \\
\text { g 3,17; mm 20; h } 12\end{array}$ \\
\hline$\frac{\text { idem }}{\left(\text { arg. }^{\text {to }}\right)}$ & $\begin{array}{l}81 \text { Similis superiori } \\
\mathrm{R}^{\circ} \%\end{array}$ & c.s. & $\begin{array}{l}\text { A1500 } \\
\text { g 2,69; mm 22,5; h } 6\end{array}$ \\
\hline$\frac{\text { idem }}{(\text { arg.t }}$ & $\begin{array}{l}\text { 82 Idem Similis } \\
\mathrm{R}^{\circ} /\end{array}$ & c.s. & $\begin{array}{l}\text { A1501 } \\
\text { g 3,79; mm 22; h } 1\end{array}$ \\
\hline \multirow[t]{2}{*}{$\begin{array}{l}\text { idem } \\
\left(\text { arg. }^{\text {to }}\right)\end{array}$} & $\begin{array}{l}83 \text { Similis. } \\
\mathrm{R}^{\circ} \%\end{array}$ & 169 Rm (251-253) & $\begin{array}{l}\text { A1482 } \\
\text { g 4,00; mm 22; h } 6\end{array}$ \\
\hline & $==$ Valerianus $==$ & & \\
\hline$\left(\arg .^{\text {to }}\right)$ & $\begin{array}{l}\text { 84. Imp. C. P. Valerianus Aug. Caput Valeriani laureatum. } \\
\text { R\%/ Apollini Conservatori. Apollo nudus s. ramum, } \\
\text { sin. lyram. }\end{array}$ & 71-72 Rm $(254-256)^{51}$ & - \\
\hline
\end{tabular}

\footnotetext{
${ }^{51}$ BESLY/BLAND 1983.
} 


\begin{tabular}{|c|c|c|c|}
\hline & & nominale/RIC/zecca/(data) & Collocazione \\
\hline$\frac{\text { idem }}{\left(\text { arg. }{ }^{\text {to }}\right)}$ & $\begin{array}{l}\text { 85. Similis superiori. } \\
\mathrm{R}^{\circ} \%\end{array}$ & c.s. & - \\
\hline$\frac{\text { idem }}{\left(\text { arg. }{ }^{\text {to }}\right)}$ & $\begin{array}{l}86 \text { Caput ut supra. } \\
\mathrm{R} \% \text { Concordia Aug. Mulier stans d. pateram super } \\
\underline{\text { aram sin. cornucopiae. }}\end{array}$ & $233 \operatorname{Vm}(254)^{52}$ & $\begin{array}{l}\text { A1529? } \\
\text { g 2,75; mm 22; h } 6\end{array}$ \\
\hline [p.] 32 & $==$ Sequitur Valerianus $==$ & & \\
\hline $\begin{array}{l}\text { idem } \\
\left(\text { arg.t }{ }^{\text {to }}\right)\end{array}$ & $\begin{array}{l}87 \text { Similis antecedenti. } \\
\mathrm{R}^{\circ} \text {. }\end{array}$ & c.s. & - \\
\hline $\begin{array}{l}\text { idem } \\
\left(\text { arg.t }{ }^{\circ}\right)\end{array}$ & $\begin{array}{l}88 \text { Caput ut supra. } \\
\text { R\%/Felicitas Aug. Mulier stans d. caduceum sin. cornucopiae. }\end{array}$ & $\begin{array}{l}282-283 \text { OR (Ant?) } \\
(256-257)^{53}\end{array}$ & - \\
\hline$\frac{\text { idem }}{\left(\text { arg. }{ }^{\text {to }}\right)}$ & $\begin{array}{l}89 \text { Caput ut supra. } \\
\mathrm{R}^{\circ} \% \text { Fides militum. Figura stans inter duo signa militaria. }\end{array}$ & $\begin{array}{l}\text { 89-90 Rm }(254-256)^{54} \\
240-241 \mathrm{Vm}(254)^{55}\end{array}$ & - \\
\hline $\begin{array}{l}\text { idem } \\
(\text { arg.to }\end{array}$ & $\begin{array}{l}\text { 90. Caput ut supra. } \\
\mathrm{R}^{\circ} \text {. Jovi Conservatori } \underline{\text { d. fulmen, sin. hastam }}\end{array}$ & $\begin{array}{lll}92-94 & R m & (254- \\
259)^{56} & & \\
\end{array}$ & - \\
\hline $\begin{array}{l}\text { idem } \\
\left(\text { arg. }^{\mathrm{to}}\right)\end{array}$ & $\begin{array}{l}\text { 91. Caput ut supra } \\
\mathrm{R}^{\circ} \text {. Oriens Aug. Solis typus, d. elata, sin. flagrum. }\end{array}$ & $\begin{array}{l}106-107 \quad \mathrm{Rm} \quad(255- \\
256)^{57}\end{array}$ & - \\
\hline $\begin{array}{l}\text { idem } \\
(\text { arg.to })\end{array}$ & $\begin{array}{l}\text { 92. Caput ut supra } \\
\mathrm{R} \% \text {. Pax Augusta. Pacis typus. }\end{array}$ & $\begin{array}{l}108-111 \text { Rm (258- } \\
259)^{58}, 245 \mathrm{Vm}(254- \\
256)^{59}\end{array}$ & - \\
\hline$\frac{\text { idem }}{\left(\text { arg. }{ }^{\circ}\right)}$ & $\begin{array}{l}93 \text { Epigraphe similis, sed posticae typus inversus a n. }{ }^{\circ} 89 . \\
\mathrm{R}^{\circ} \%\end{array}$ & \begin{tabular}{|l|}
$89-90 \mathrm{Rm}(254-256)^{60}$ \\
$240-241 \mathrm{Vm}(254)^{61}$
\end{tabular} & - \\
\hline$\frac{\text { idem }}{\left(\text { arg.t }{ }^{\mathrm{to}}\right)}$ & $\begin{array}{l}\text { 94. Caput ut supra. } \\
\mathrm{R} \% \text { Salus Aug. Mulier ut videtur serpentem pascit in } \\
\text { patera super aram. }\end{array}$ & \begin{tabular}{|l|} 
Probailmente ascri- \\
vibile a Gallieno RIC \\
397A Vm $(254-256)^{62}$ \\
\end{tabular} & $\begin{array}{l}\text { B5719 } \\
\text { g 3,61; mm 21; h } 6\end{array}$ \\
\hline$\frac{\text { idem }}{\left.\text { (arg.t }{ }^{\circ}\right)}$ & 95. Nummis similis $n .^{\circ} 79$. & $\begin{array}{l}\text { MIR, 36, n. } 811 \mathrm{Vm} \\
(253-256)^{63}\end{array}$ & $\begin{array}{l}\text { B5518 } \\
\text { g 3,42; mm 22; h } 6\end{array}$ \\
\hline [p.] 33. & $==$ Sequitur Valerianus $=\equiv$ & & \\
\hline $\begin{array}{l}\text { idem } \\
\left(\text { arg. } .^{\text {to }}\right)\end{array}$ & $\begin{array}{l}\text { 96. Similis antecedenti. } \\
\mathrm{R}^{\circ} \%\end{array}$ & c.s. & $\begin{array}{l}\text { B5519 } \\
\text { g 3,67; mm 22,5; h } 12\end{array}$ \\
\hline$\frac{\text { idem }}{\left(\text { arg. }^{\text {to }}\right)}$ & $\begin{array}{l}\text { 97. Idem similis } \\
\mathrm{R}^{\circ} /\end{array}$ & c.s. & $\begin{array}{l}\text { B5521 } \\
\text { g 2,48; mm 22; h } 6\end{array}$ \\
\hline$\frac{\text { idem }}{\left.\text { (arg. }{ }^{\text {to }}\right)}$ & $\begin{array}{l}\text { 98. Similis. } \\
\mathrm{R} \%\end{array}$ & $266 \operatorname{Vm}(253)^{64}$ & $\begin{array}{l}\text { B5516 } \\
\text { g 3,51; mm 22; h } 6\end{array}$ \\
\hline $\begin{array}{l}\text { idem } \\
\left(\text { arg. }^{\text {to }}\right)\end{array}$ & $\begin{array}{l}99 \text { Similis. } \\
\mathrm{R}^{\circ} \%\end{array}$ & $267 \mathrm{Vm}(253)^{65}$ & $\begin{array}{l}\text { B5517 } \\
\text { g 3,57; mm 21; h } 6\end{array}$ \\
\hline
\end{tabular}

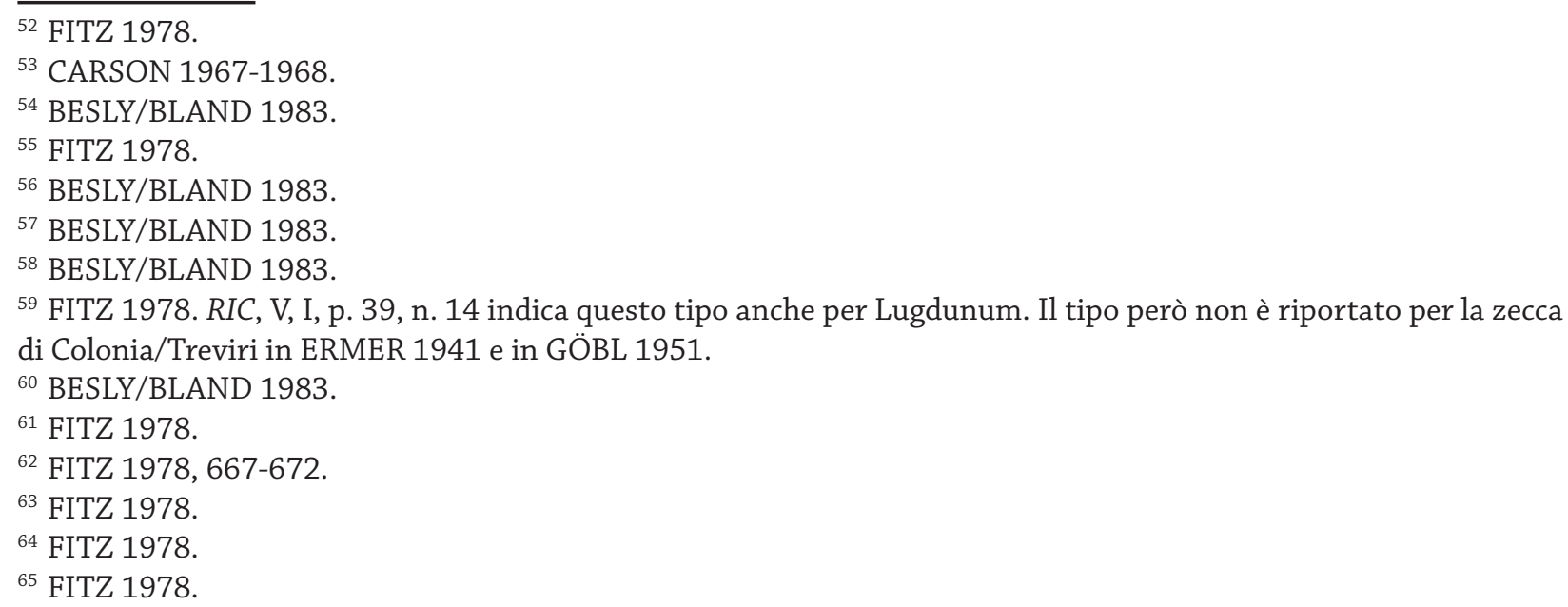




\begin{tabular}{|c|c|c|c|}
\hline & & nominale/RIC/zecca/(data) & Collocazione \\
\hline$\frac{\text { idem }}{\left(\text { arg.t }{ }^{\text {to }}\right)}$ & $\begin{array}{l}100 \text { Caput ut supra. } \\
\mathrm{R}^{\circ} \% \text { Victoria Augg. Typus Victoriae, d. coronam. }\end{array}$ & $\begin{array}{l}\text { 123-124 Rm }(254)^{66}, \\
223-224 \mathrm{Ant}(254)^{67} \\
260 \mathrm{Vm}(257-258)^{68}\end{array}$ & - \\
\hline $\begin{array}{l}\text { idem } \\
\left(\text { arg. }^{\text {to }}\right)\end{array}$ & $\begin{array}{l}\text { 101. Fere similis superiori, sed d. scuto innixa. } \\
\mathrm{R}^{\circ} \%\end{array}$ & $\begin{array}{l}127-128 \quad R m \quad(254- \\
256)^{69}, 261 \text { Ant }(255)^{70}\end{array}$ & - \\
\hline$\frac{\text { idem }}{\left(\text { arg. }{ }^{\circ}\right)}$ & $\begin{array}{l}\text { 102. Similis n. }{ }^{\circ} 92 . \\
\mathrm{R}^{\circ} \%\end{array}$ & $\begin{array}{l}108-111 \quad \mathrm{Rm} \quad(258- \\
259)^{71}, 245 \mathrm{Vm}(254- \\
256)^{72}\end{array}$ & - \\
\hline \multirow[t]{2}{*}{$\frac{\underline{\text { idem }}}{\left(\text { arg.t }{ }^{\text {to }}\right)}$} & $\begin{array}{l}\text { 103. Similis n. }{ }^{\circ} 89 . \\
\mathrm{R}^{\circ} /\end{array}$ & 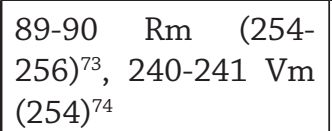 & - \\
\hline & $==\mathrm{P}$. Licinius $\underline{\text { Gallienus }}==$ & & \\
\hline $\begin{array}{l}\text { idem } \\
\left(\text { arg.t }^{\mathrm{t}}\right)\end{array}$ & $\begin{array}{l}\text { 104. Imp. Gallienus Pius Aug. Caput } \\
\text { R\%. Virtus Aug. Figura stans d. hastam sin. scuto in- } \\
\text { nixa. }\end{array}$ & $\begin{array}{|llr|}181-183 & \mathrm{Rm} & (254- \\
256)^{75}, & 410 & \mathrm{Vm} \\
(254-256)^{76}, 301 & \mathrm{Ant} \\
(254)^{77}, & * 325 & \mathrm{Rm} \\
(261-263), & 531-536 \\
\text { Med }(262-263), & 667- \\
668 \text { Ant } & (263-264)\end{array}$ & - \\
\hline$\frac{\text { idem }}{\left(\text { arg. }{ }^{\circ}\right)}$ & $\begin{array}{l}105 \text { Caput ut supra. } \\
\text { R०\% Aeternitas Aug. Typus Solis manu elata, sin. glo- } \\
\text { bum, in area } \Gamma \text {. }\end{array}$ & *160 Rm (266) & - \\
\hline [p.] $\underline{34}$. & $==$ Sequitur Valerianus Gallienus $==$ & & \\
\hline $\begin{array}{l}\text { idem } \\
\left(\text { arg.t }^{\text {to }}\right)\end{array}$ & $\begin{array}{l}\text { 106. Caput ut supra. } \\
\mathrm{R}^{\circ} \text {. Diana Felix. Diana succincta stans, d. hastam } \\
\text { sin. arcum cum cane venatico ad pedes. }\end{array}$ & \begin{tabular}{|l|}
$380 \quad$ Med $(259-$ \\
$260), * 473$ Med (262- \\
$263)$
\end{tabular} & - \\
\hline $\begin{array}{l}\text { idem } \\
\left(\text { arg.t }{ }^{\circ}\right)\end{array}$ & $\begin{array}{l}\text { 107. Caput ut supra } \\
\mathrm{R}^{\circ} \% \text { Postica similis } \mathrm{n} .^{\circ} 80 .\end{array}$ & $\begin{array}{|lrr|}375 \quad \text { Tre/Vm } & (253- \\
260)^{78}, \quad * 471 & \text { Med } \\
(267), 562 & \text { Sis } & (266- \\
268)^{79} & \end{array}$ & - \\
\hline
\end{tabular}

\footnotetext{
${ }^{66}$ BESLY/BLAND 1983.

${ }^{67}$ CARSON 1967-1968.

${ }^{68}$ FITZ 1978.

${ }^{69}$ BESLY/BLAND 1983.
}

${ }^{70}$ CARSON 1967-1968. RIC, V, I, p. 39, n. 21 indica in tipo con legenda VICT AVGG anche per Lugdunum. Il tipo però non è riportato per la zecca di Colonia/Treviri in ELMER 1941, GÖBL 1951, MIR, 36.

${ }^{71}$ BESLY/BLAND 1983.

${ }^{72}$ FITZ 1978. RIC, V, I, p. 39, n. 14 indica questo tipo anche per Lugdunum. Il tipo però non è riportato per la zecca di Colonia/Treviri in ERMER 1941 e GÖBL 1951.

${ }^{73}$ BESLY/BLAND 1983.

${ }^{74}$ FITZ 1978.

${ }^{75}$ BESLY/BLAND 1983.

${ }^{76}$ FITZ 1978. RIC, V, I, p. 72, n. 59 indica un tipo con legenda VIRTVS AVGG per Lugdunum. Il tipo però non è riportato per la zecca di Colonia/Treviri in ERMER 1941, in GÖBL 1951, MIR, 36.

${ }^{77}$ CARSON 1967-1968.

${ }^{78}$ RIC, V, I, p. 98, n. 375 riporta un antoniniano con legenda CONCOR AVG del tipo indicato nel manoscritto, riferibile alla zecca di Mediolamun e ascrivibile a Gallieno nella fase del regno congiunto con Valeriano: al dritto sarebbe presente la legenda IMP GALLIENVS P AVG. Il tipo però non è riportato in GÖBL 1951, MIR, 36. Tuttavia in questo caso potrebbe trattarsi di una emissione inedita o di un un ibrido riferibile alle zecche di Viminacium o, meno probabilmente, di Treviri, dove quella legenda del dritto è annoverata nel periodo indicato, benché il tipo del rovescio non sia elencato né per Gallieno, né per Valeriano; tuttavia l'abbinamento è noto per Valeriano con legenda parallela a quella detta (IMP VALERIANVS P AVG) grazie a un'asta recente: iNumis, Mail Bid Sale 11, 19 March 2010, 


\begin{tabular}{|c|c|c|c|}
\hline & & nominale/RIC/zecca/(data) & Collocazione \\
\hline $\begin{array}{l}\text { idem } \\
\left(\text { arg. }^{\text {to }}\right)\end{array}$ & $\begin{array}{l}108 \text { Similis } n^{\circ} 91 . \\
\mathrm{R}^{\circ} \%\end{array}$ & $\begin{array}{l}154 \mathrm{Rm}(255-256)^{80} \\
{ }^{8} 248-249 \mathrm{Rm}(266) \\
494 \mathrm{Med}(263)\end{array}$ & - \\
\hline$\frac{\text { idem }}{\left(\text { arg. }^{\text {to }}\right)}$ & $\begin{array}{l}\text { 109. Caput ut supra. } \\
\mathrm{R}^{\circ} \text {. Jovi Statori. Figura nuda stans d. hastam, sin. elata. }\end{array}$ & $\begin{array}{ll}\text { Probabilmente } & \text { Iovi } \\
\text { Conservat }{ }^{*} 210 & \mathrm{Rm} \\
(266) & \end{array}$ & $\begin{array}{l}\text { B5636 } \\
\text { g 3,10; mm 20; h } 12\end{array}$ \\
\hline$\left.\frac{\text { idem }}{(\text { arg.to }}\right)$ & $\begin{array}{l}110 \text { Caput ut supra. } \\
\mathrm{R}^{\circ} / \text { Marti Pacifero. Figura militaris stans d. ramum, } \\
\text { sin. hastam et scutum. In area R [i.e. A, n.d.r.]. }\end{array}$ & *236 Rm (266) & - \\
\hline$\frac{\text { idem }}{\left(\text { arg. }{ }^{\circ}\right)}$ & $\begin{array}{l}111 \text { Caput ut supra. } \\
\text { R}^{\circ} / \text { Salus Augusta. Serpetem pascit in pateram sin. } \\
\text { cornucopiae. }\end{array}$ & *512a Med (267) & $\begin{array}{l}\text { B5718 } \\
\text { g 2,63; mm 22; h } 6\end{array}$ \\
\hline$\left.\frac{\text { idem }}{(\text { arg.to }}\right)$ & $\begin{array}{l}112 \text { Caput ut supra. } \\
\mathrm{R}^{\circ} \% \text { Uberitas } \underline{\text { Sic Aug. Figura stans d. demissa spicas, }} \\
\text { sin. cornucopiae. }\end{array}$ & $\begin{array}{l}\text { Probabilmente con- } \\
\text { fuso con Treboniano } \\
\text { Gallo RIC } 92^{81} \text { Ant } \\
(251-253)\end{array}$ & $\begin{array}{l}\text { A1437 } \\
\text { g 3,51; mm 22; h } 12\end{array}$ \\
\hline $\begin{array}{l}\text { idem } \\
\left(\text { arg.t }{ }^{\mathrm{to}}\right)\end{array}$ & $\begin{array}{l}\text { 113. Caput ut supra } \\
\mathrm{R}^{\circ} \text { Epigraphe incerta. Figura stans d. baculum de- } \\
\text { missum, sin. hastam. }\end{array}$ & $?$ & - \\
\hline$\left.\frac{\text { idem }}{(\text { arg.to }}\right)$ & $\begin{array}{l}\text { 114. Caput ut supra } \\
\mathrm{R}^{\circ} \text { Epigraphe deleta. Figura stans d. demissa sin. } \\
\text { cornucopiae. }\end{array}$ & $?$ & - \\
\hline [p.] $\underline{35}$. & $==$ Sequitur Gallienus $==$ & & \\
\hline $\begin{array}{l}\text { idem } \\
\left(\text { arg.t }{ }^{\circ}\right)\end{array}$ & $\begin{array}{l}\text { 115. Caput ut supra. } \\
\mathrm{R}^{\circ} \text { Epigraphe incerta. }\end{array}$ & $?$ & - \\
\hline $\begin{array}{l}\text { idem } \\
\left(\text { arg. }{ }^{\text {to }}\right)\end{array}$ & $\begin{array}{l}\text { 116. Caput ut supra. } \\
\mathrm{R}^{\circ} \text {. Victoria d. coronam, sin. palmam. }\end{array}$ & $?$ & - \\
\hline $\begin{array}{l}\text { idem } \\
\left(\text { arg. }^{\text {to }}\right)\end{array}$ & $\begin{array}{l}117 \text { Fere similis superiori. } \\
\mathrm{R}^{\circ} \%\end{array}$ & $?$ & - \\
\hline$\frac{\text { idem }}{\left(\text { arg. }^{\text {to }}\right)}$ & $\begin{array}{l}118 \text { Fere similis superiori } \\
\mathrm{R} \%\end{array}$ & $?$ & - \\
\hline$\left.\frac{\text { idem }}{(\text { arg. }}{ }^{\text {to }}\right)$ & $\begin{array}{l}\text { 119. Simils n. } 71 \text {. } \\
\mathrm{R}^{\circ} \%\end{array}$ & cfr. supra 104 & - \\
\hline \multirow[t]{2}{*}{$\begin{array}{l}\text { idem } \\
\left(\text { arg. }^{\text {to }}\right)\end{array}$} & $\begin{array}{l}120 \text { Similis superiori. } \\
\mathrm{R}^{\circ} \text {. }\end{array}$ & c.s. & - \\
\hline & $==$ Cornelia $\underline{\text { Salonina }}==$ & & \\
\hline$\left(\arg \cdot{ }^{\text {to }}\right)$ & $\begin{array}{l}121 \text { Cornelia Salonina Aug. Caput. ejusdem. } \\
\text { R\% Pietas Aug. Stans d. elata } \sin . . .\end{array}$ & *22 Rm (263) & - \\
\hline$\frac{\text { eadem }}{\left(\text { arg.t }{ }^{\circ}\right)}$ & $\begin{array}{l}122 \text { Caput ut supra } \\
\mathrm{R} \% \text { Epigraphe fere deleta. Mulier sedens d. demissa, } \\
\text { sin. hastam. }\end{array}$ & $?$ & - \\
\hline $\begin{array}{l}\text { eadem } \\
\left(\text { arg. }^{\text {to }}\right)\end{array}$ & $\begin{array}{l}\text { 123. Caput ut supra. } \\
\mathrm{R}^{\circ} \% \text { Concordia Aug. Duae figurae jungentes dexteras. }\end{array}$ & $\begin{array}{l}63 \text { OR (Cyz?) }(256- \\
259)^{82}\end{array}$ & - \\
\hline
\end{tabular}

lotto n. 164. Questo elemento rende plausibile che la moneta riportata in RIC sia la corrispondente a quest'ultima di Valeriano e che dunque vi sia una coniazione anche per Gallieno con la tipologia CONCOR AVGG e raffigurazione della Concordia seduta con patera e cornucopia, durante il regno congiunto. In questa eventualità la moneta indicata nel manoscritto potrebbe essere datata anche nel periodo 253-260 d.C.

${ }^{79} \mathrm{MIR}, 36,121-122$.

${ }^{80}$ BESLY/BLAND 1983.

${ }^{81}$ Catalogato come RIC 80 in ASOLATI/CRISAFULLI 1996.

${ }^{82}$ CARSON 1967-1968, 129-130, 140-141. 


\begin{tabular}{|c|c|c|c|}
\hline & & nominale/RIC/zecca/(data) & Collocazione \\
\hline$\frac{\text { eadem }}{(\text { arg.t. }}$ & $\begin{array}{l}124 \text { Caput ut supra. } \\
\mathrm{R}^{\circ} \text {. Fecunditas Aug. Mulier stans d. puerulo porri- } \\
\text { gens, sin. alium puerulum tenens. }\end{array}$ & $\begin{array}{l}26, \quad 57 \\
260)^{83}\end{array}$ & - \\
\hline [p.] $\underline{36}$ & $==$ Sequitur Cornelia $\underline{\text { Salonina }}==$ & & \\
\hline$\left(\right.$ arg. $\left.{ }^{\text {to }}\right)$ & $\begin{array}{l}\text { 125. Caput ut supra } \\
\mathrm{R}^{\circ} \text { / Pietas Aug. Figura muliebris sedens, sin. hastam } \\
\text { puram, ad pedes duo pueruli }\end{array}$ & $35 \mathrm{Rm}(256)^{84}$ & - \\
\hline$\frac{\text { eadem }}{\left(\text { arg. }{ }^{\circ}\right)}$ & $\begin{array}{l}126 \text { Caput ut supra } \\
\mathrm{R} \% \text {. Postica fere deleta. }\end{array}$ & ? & - \\
\hline$\frac{\text { eadem }}{\left(\text { arg. }{ }^{\circ}\right)}$ & $\begin{array}{l}\text { 127. Caput ut supra } \\
\mathrm{R} \% \text {....mulier stans.... }\end{array}$ & $?$ & - \\
\hline$\frac{\text { eadem }}{\left(\text { arg. }{ }^{\circ}\right)}$ & $\begin{array}{l}\text { 128. Caput ut supra. } \\
\mathrm{R} \% \text { Venus Felix. Mulier sedens, d. pateram cum co- } \\
\text { lumba, sin. hastam. }\end{array}$ & 7 Tre $(25785$ & - \\
\hline$\frac{\text { eadem }}{\left(\text { arg. }{ }^{\circ}\right)}$ & $\begin{array}{l}\text { 129. Caput ut supra. } \\
\mathrm{R} \% \text { Epigraphe incerta. Mulier sedens d. ramum, sin. } \\
\text { hastam. }\end{array}$ & $\begin{array}{l}\text { *57-60 Med (261- } \\
267)\end{array}$ & - \\
\hline
\end{tabular}

\footnotetext{
${ }^{83}$ BESLY/BLAND 1983, 32.

${ }^{84}$ GÖBL 1951, 22.

${ }^{85}$ GÖBL 1951, 31.
} 
Cartella A

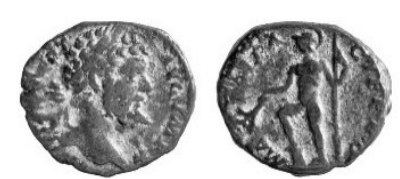

2
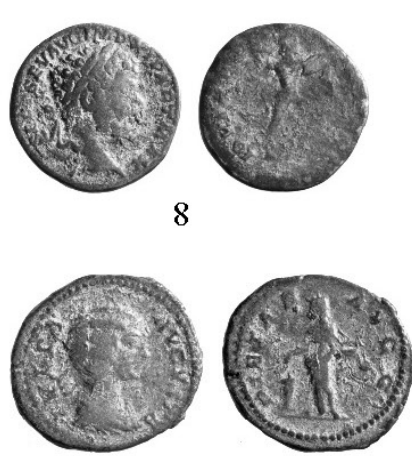

11

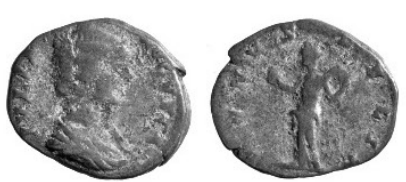

14

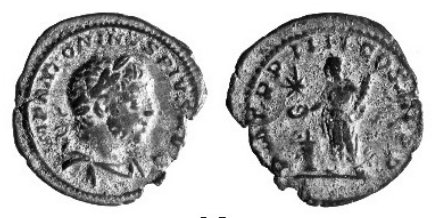

28

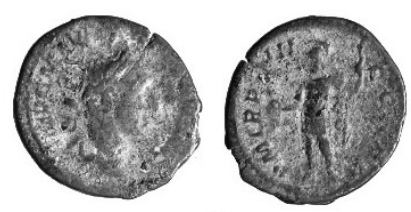

40

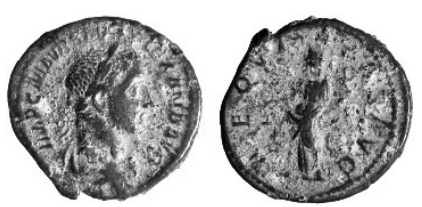

44

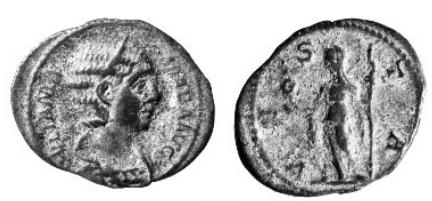

51

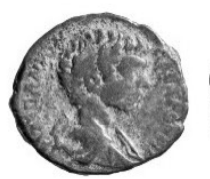

16

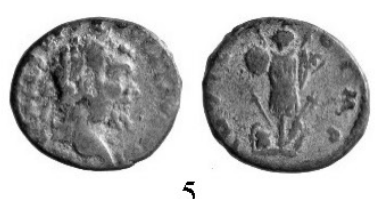

5

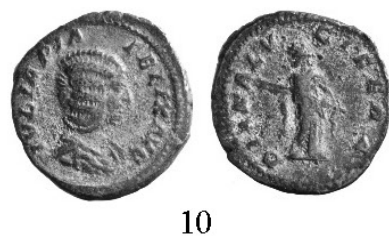

9
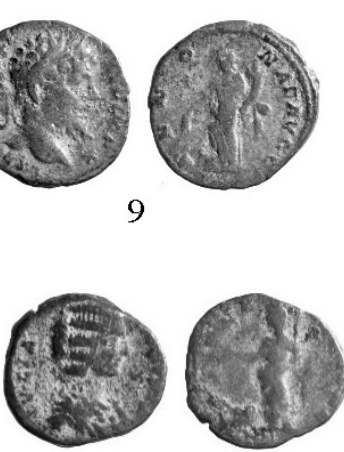

12

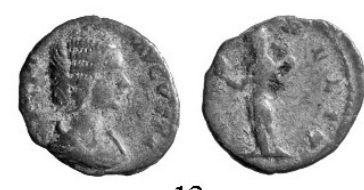

13

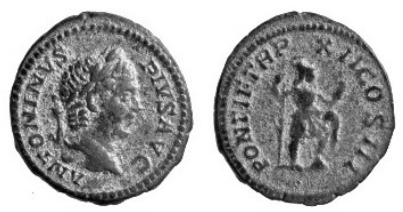

18

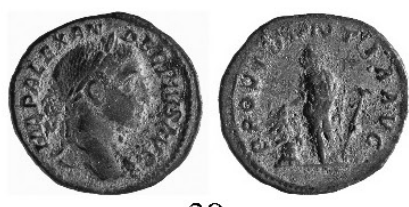

39

32
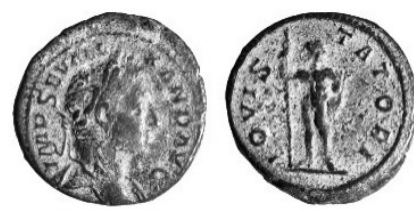

42

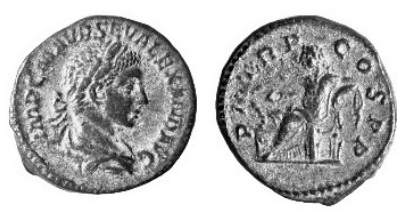

43

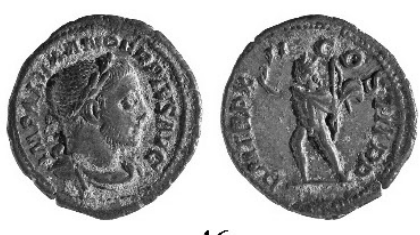

46

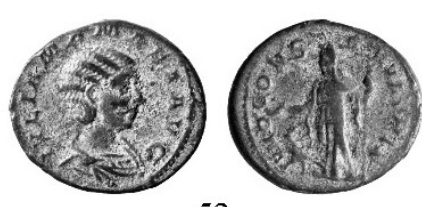

53

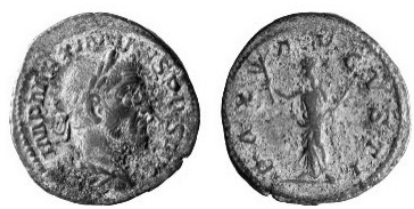

57 

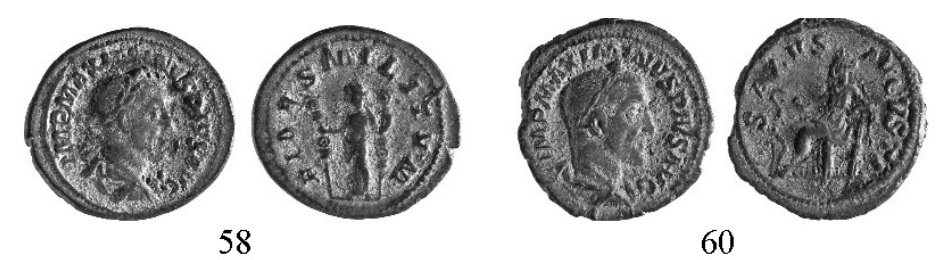

Cartella B
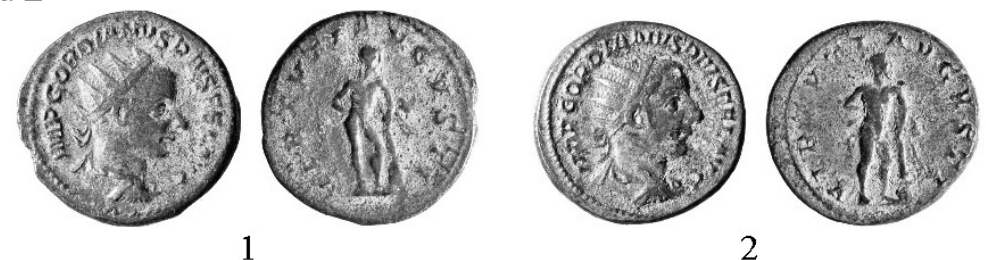

2

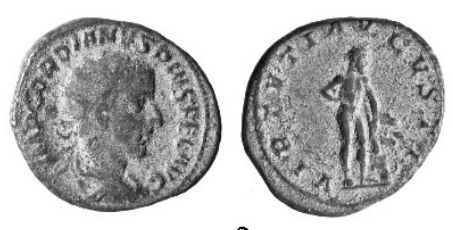

3
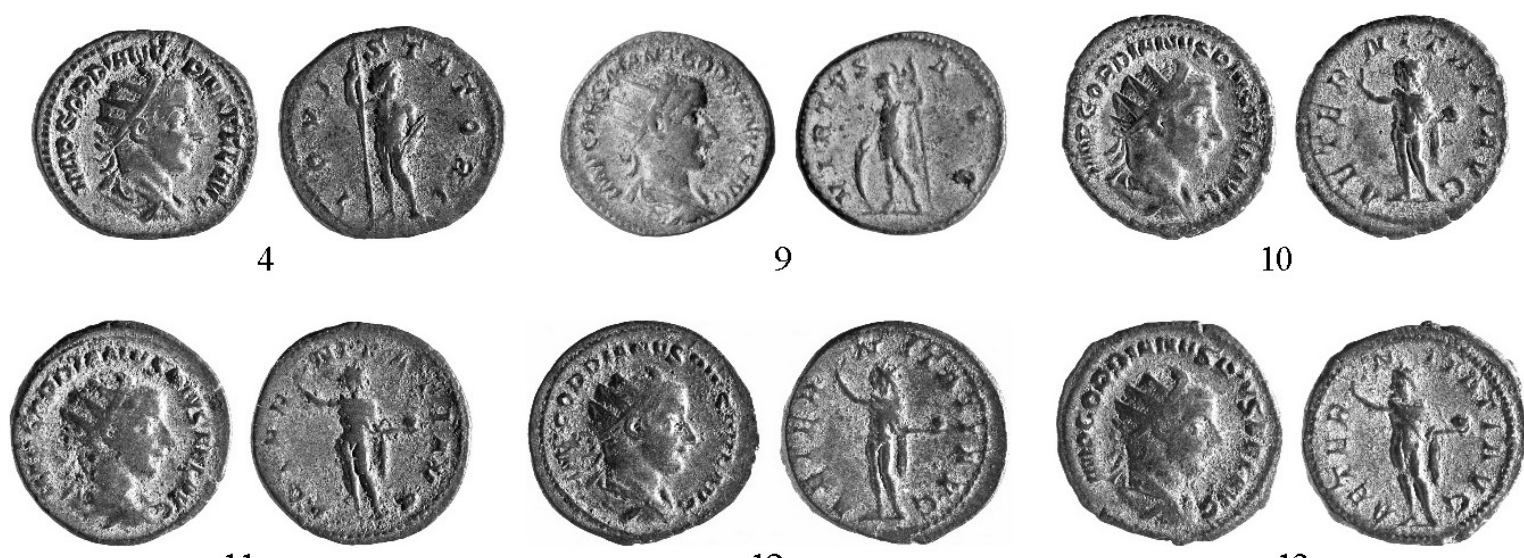

12
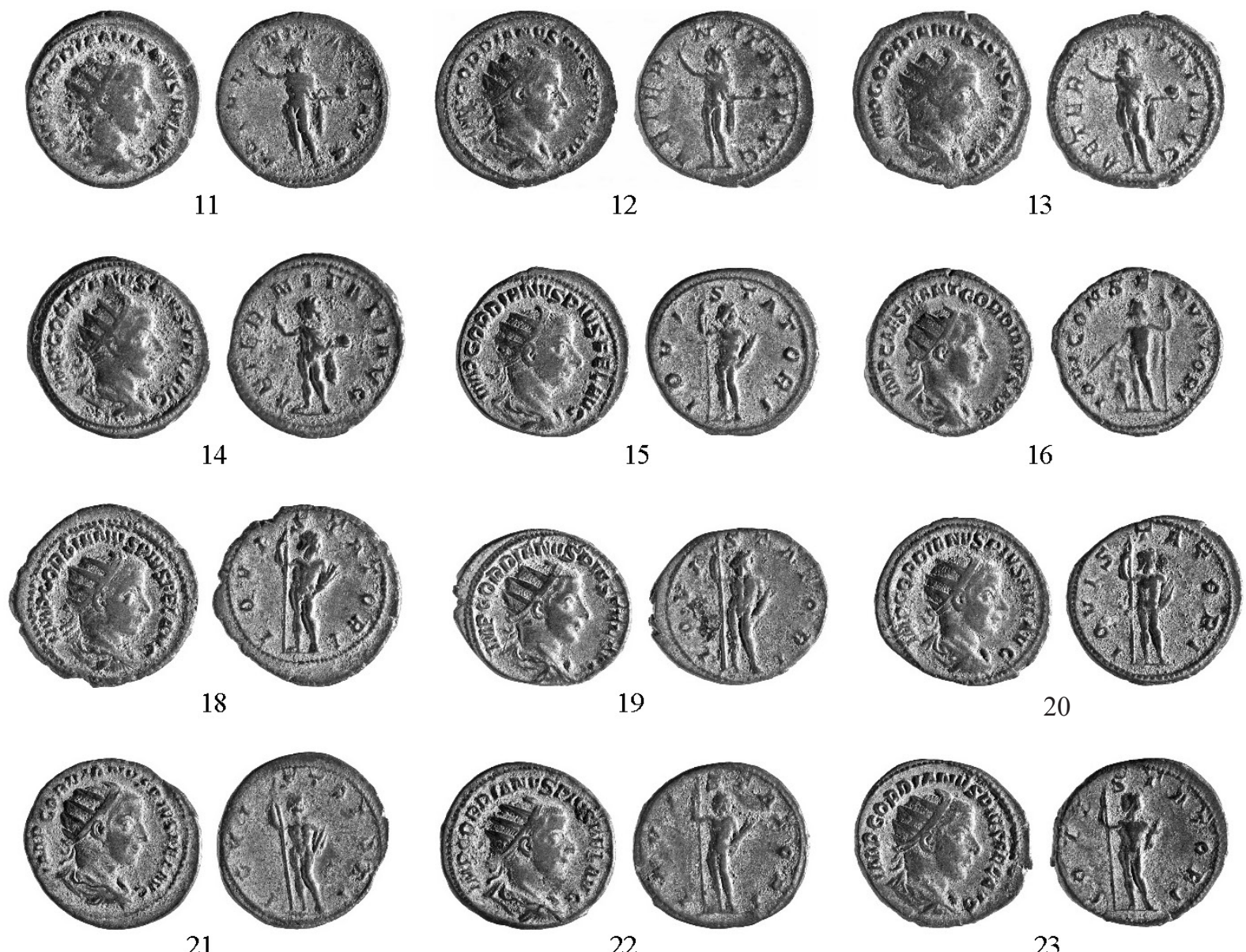

22

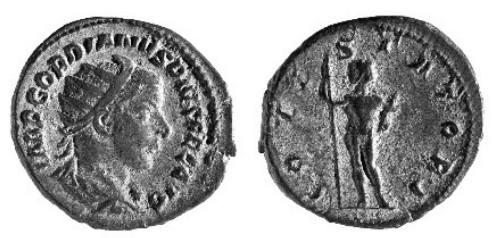

23

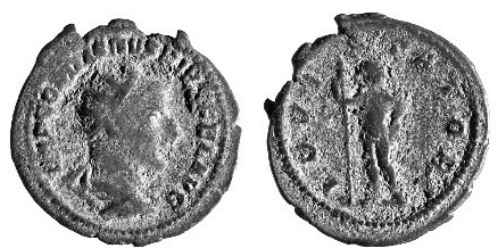

24

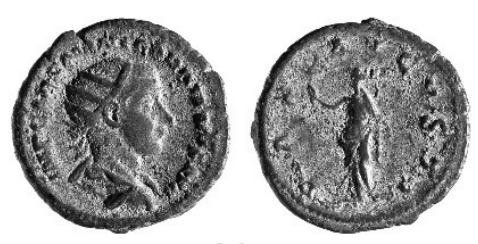

26

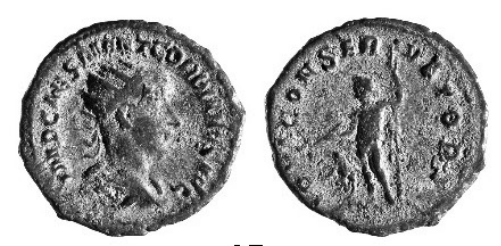




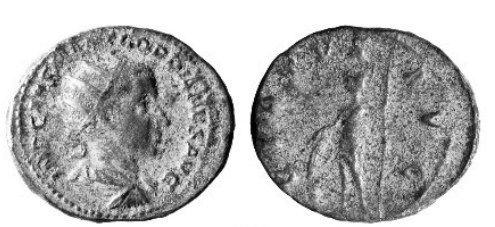

28

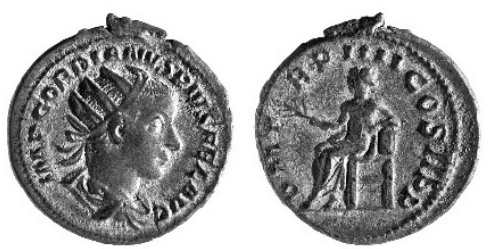

32

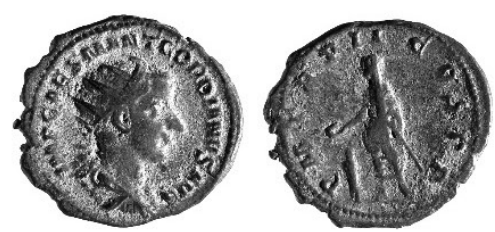

40

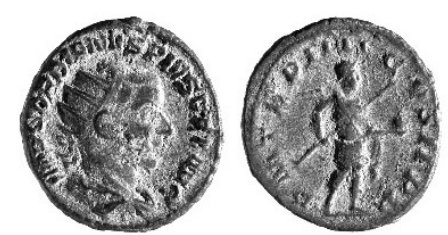

43

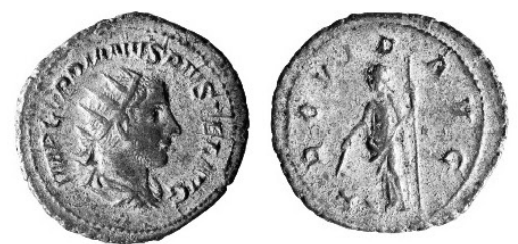

46

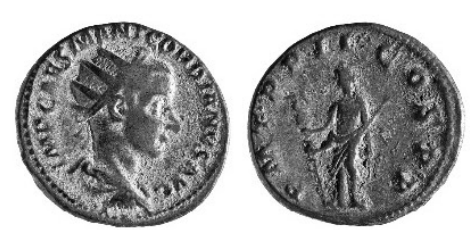

50

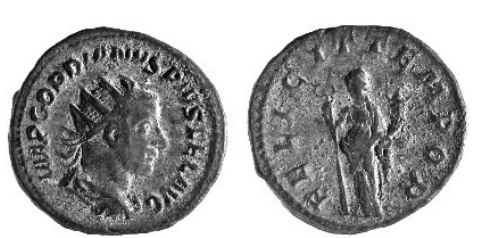

54

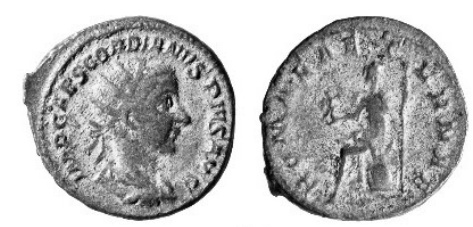

57

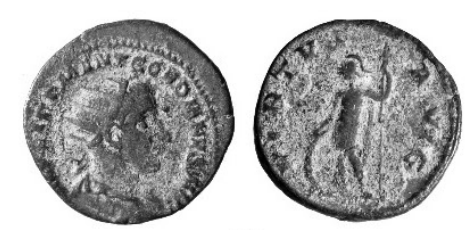

29

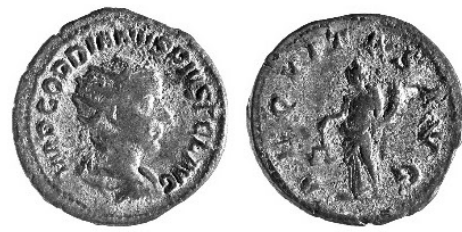

37

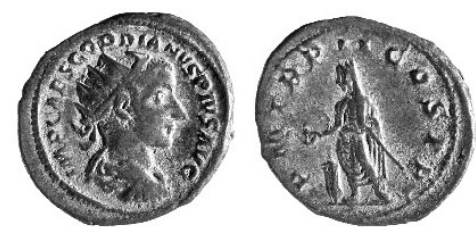

41

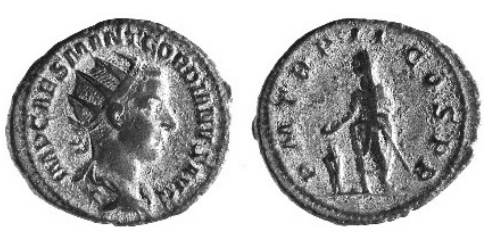

39

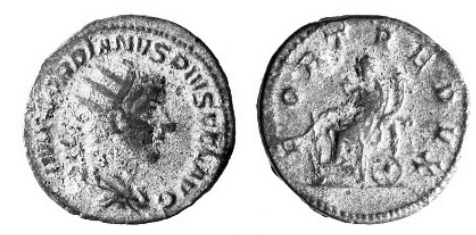

42

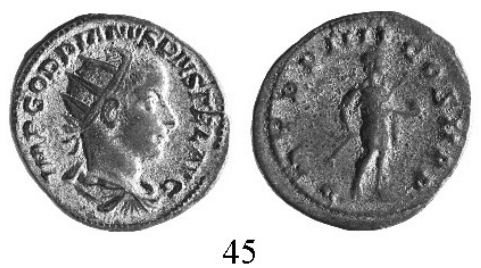

44
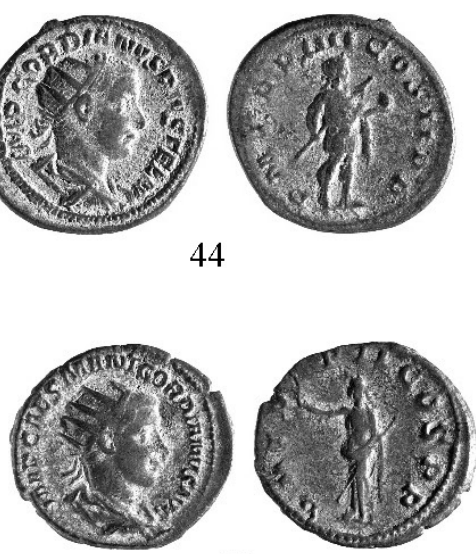

47

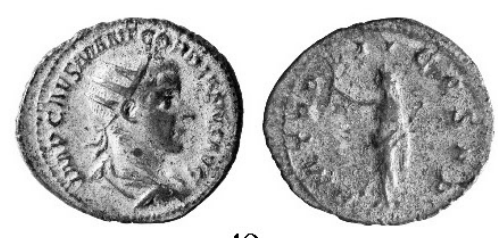

49

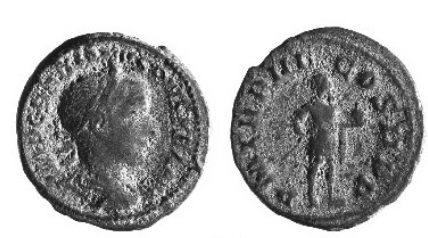

52

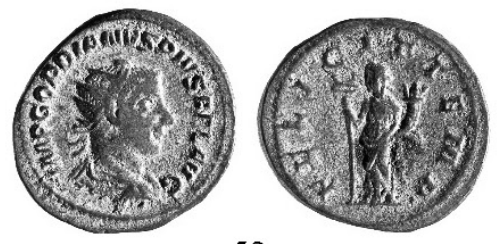

53

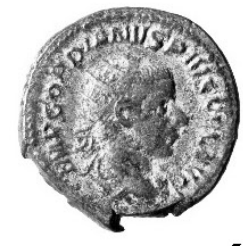

55
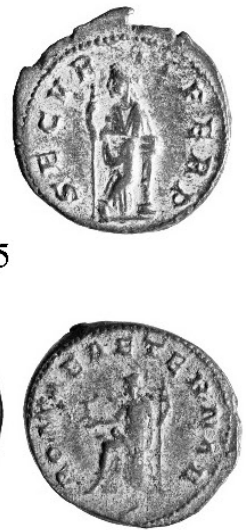

58
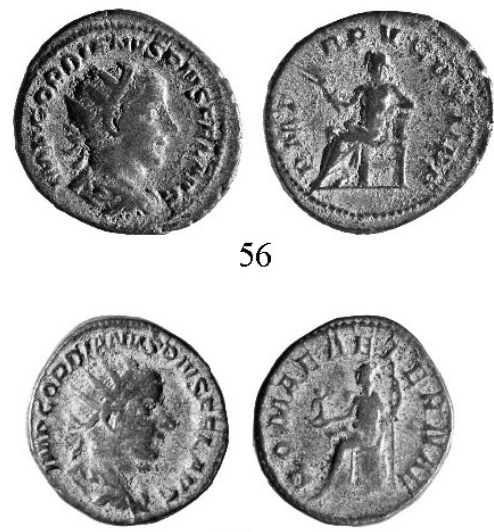

59 


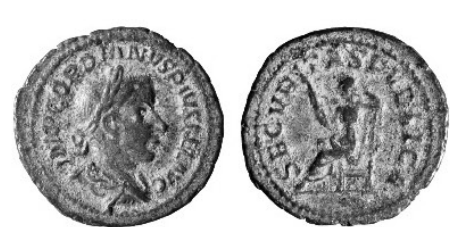

63

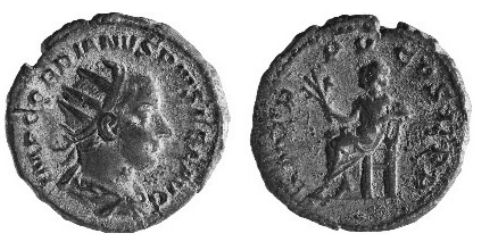

67

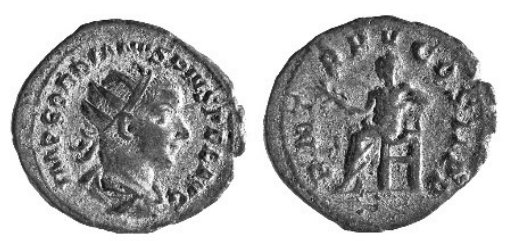

65

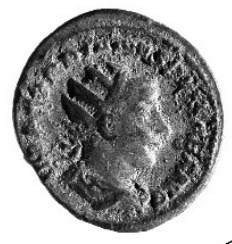

66

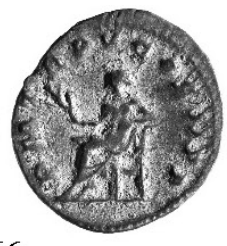

66

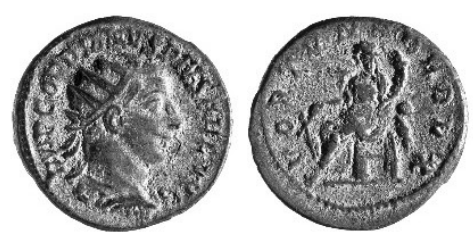

69

Cartella C
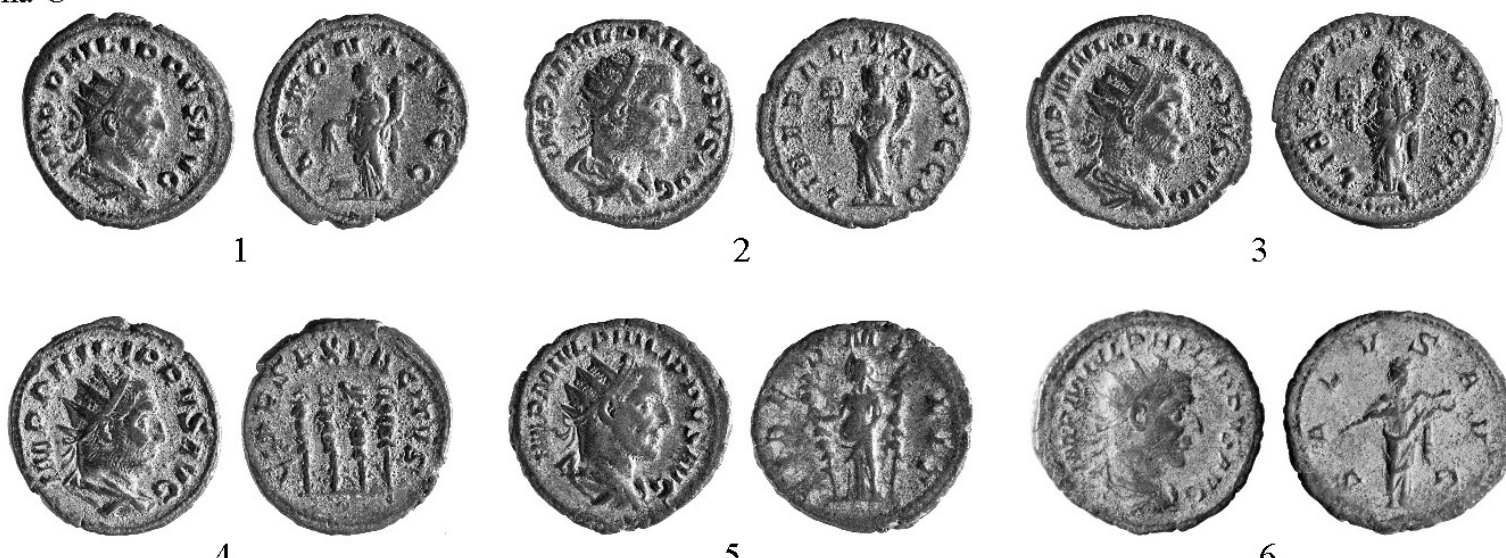

4
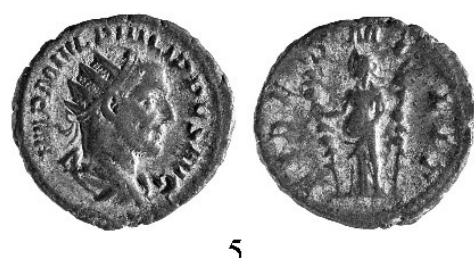

5
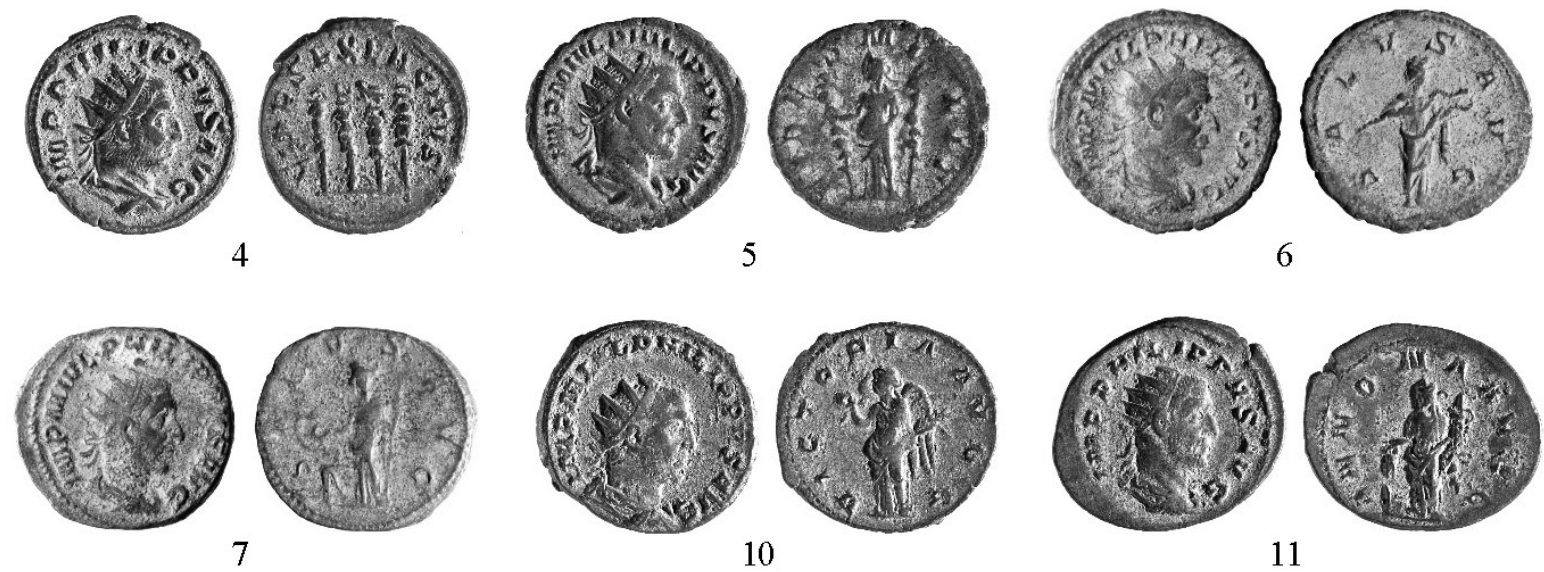

10
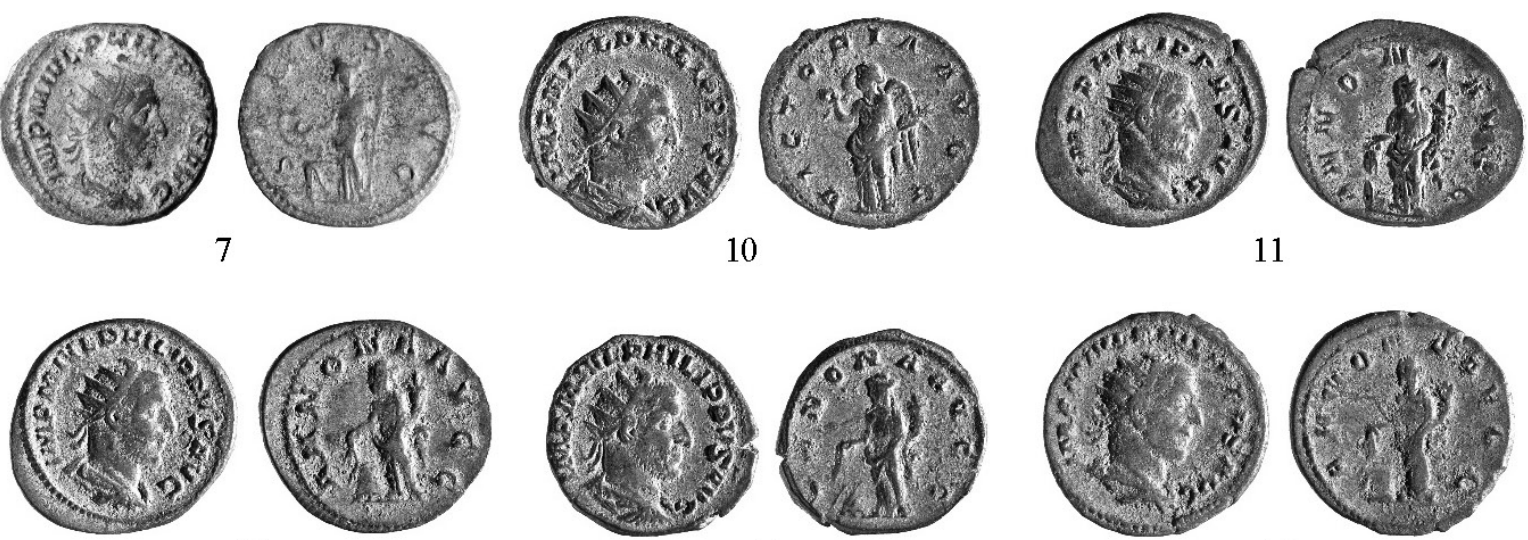

13

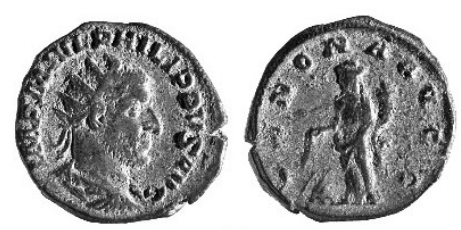

14

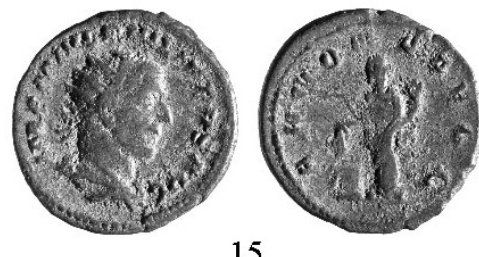

15

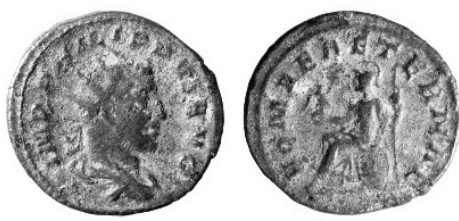

19

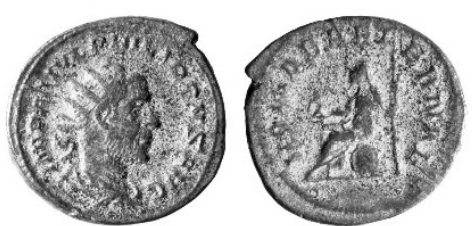

22

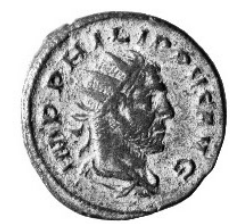

20
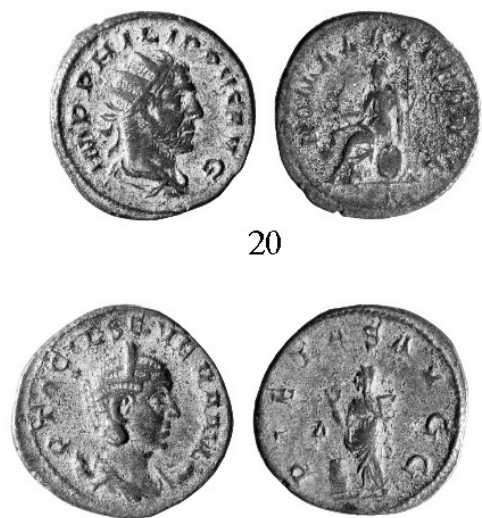

27

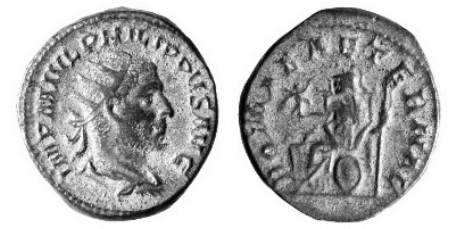

21

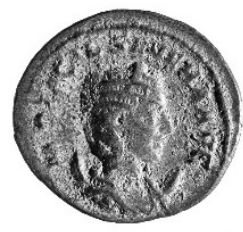

28 


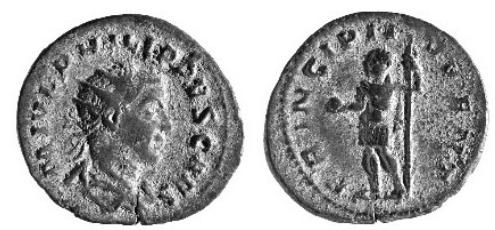

29

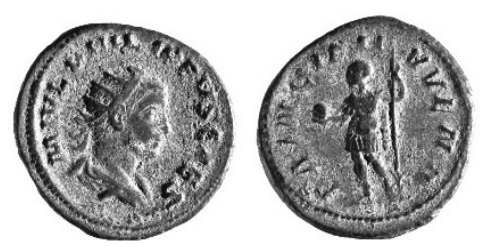

34

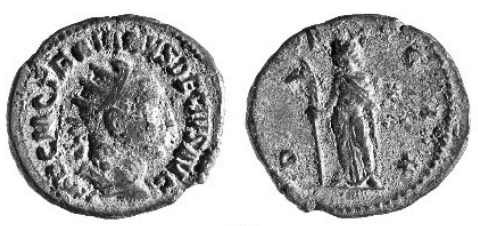

38

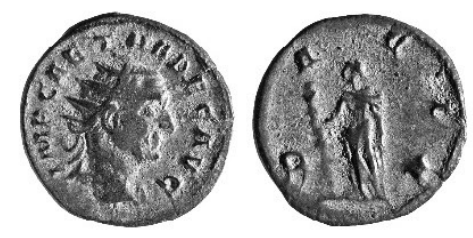

42

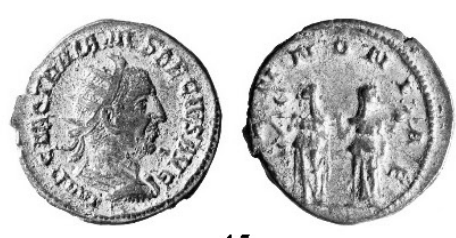

45

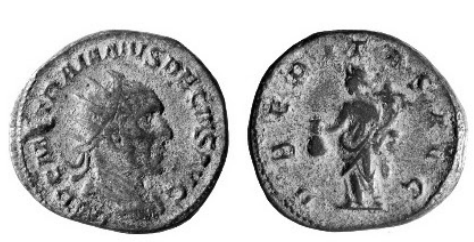

53

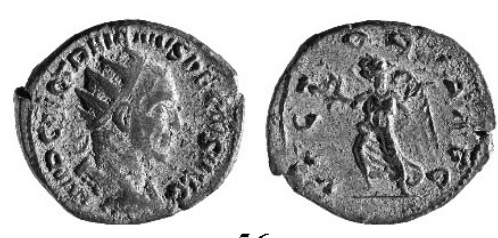

56

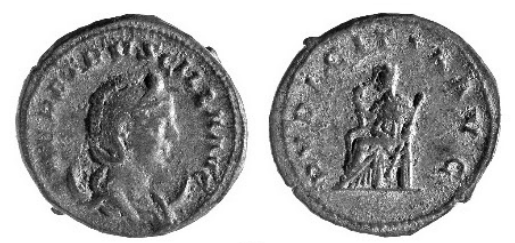

61

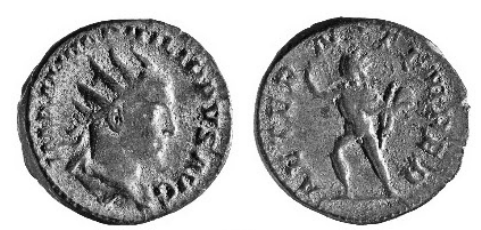

31

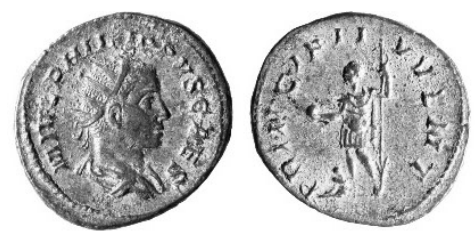

35

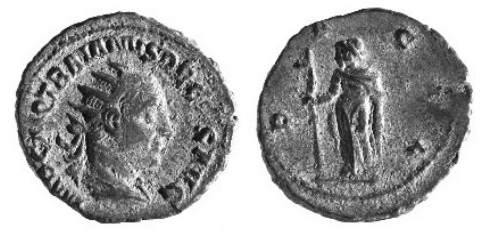

39

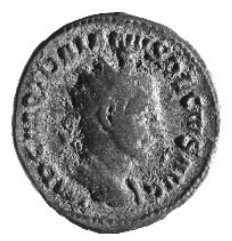

43

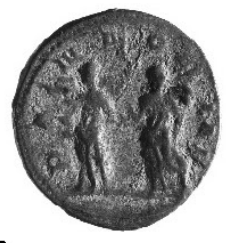

.

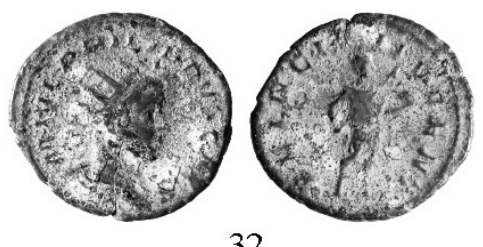

32

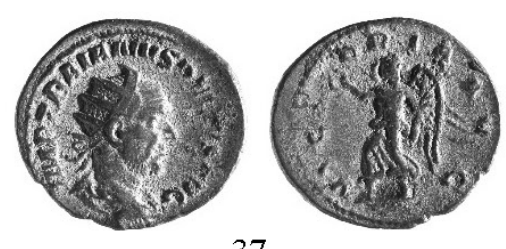

37

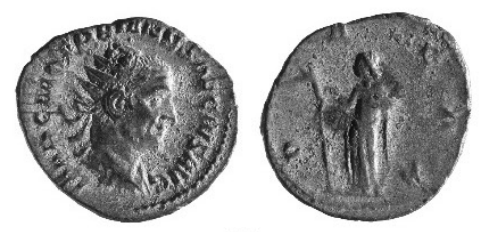

40

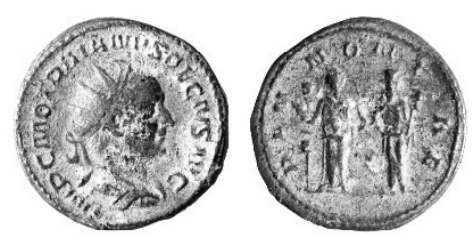

44

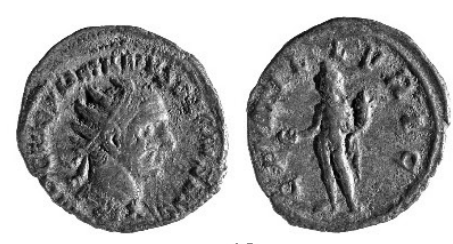

49

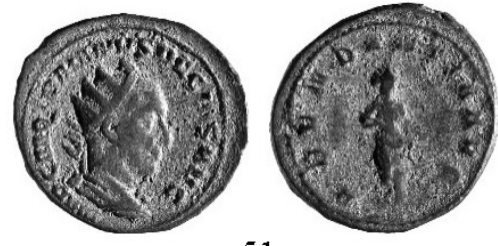

51

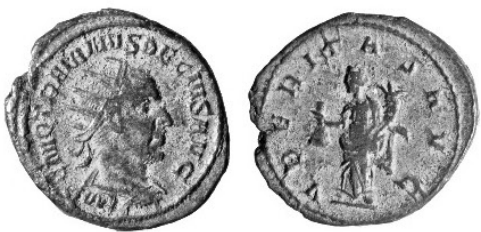

55

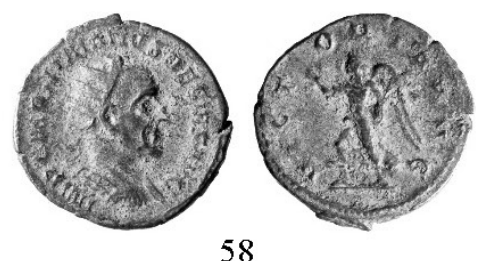

57

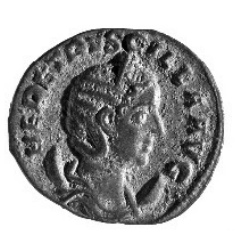

62
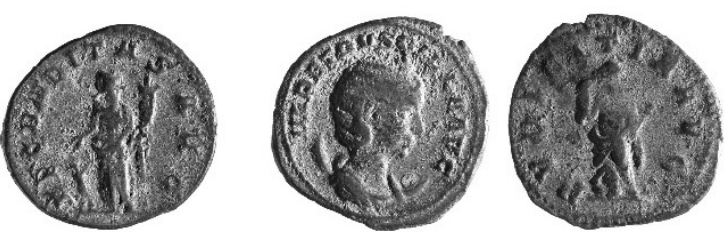

63 


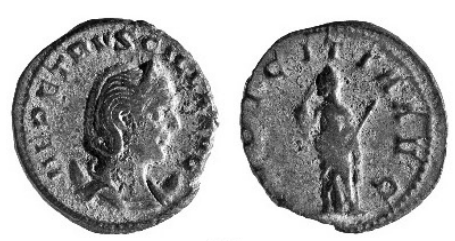

64

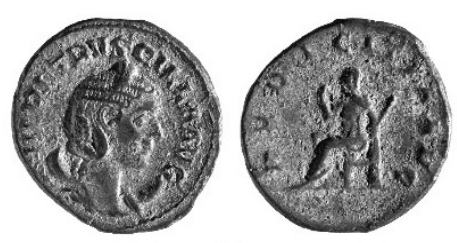

67

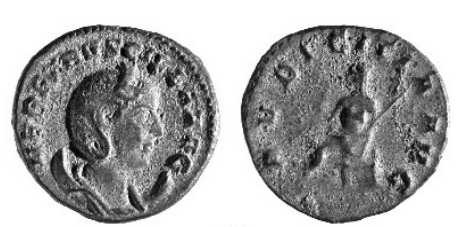

65

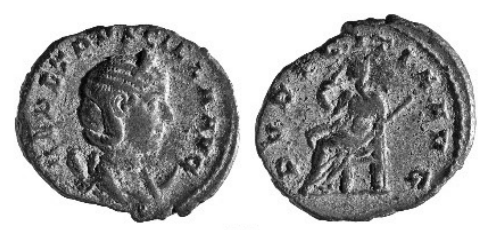

66

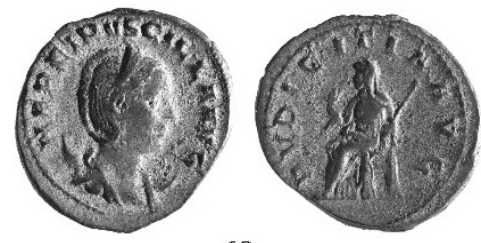

68

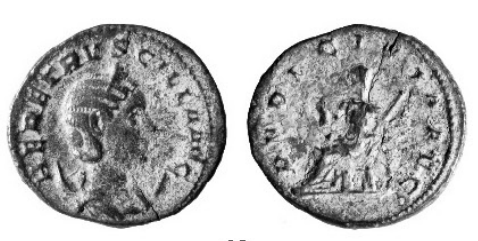

69

Cartella D

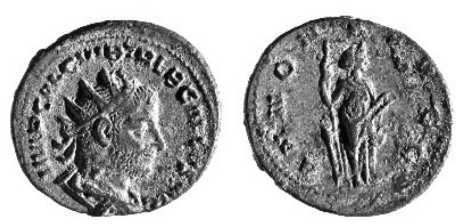

3

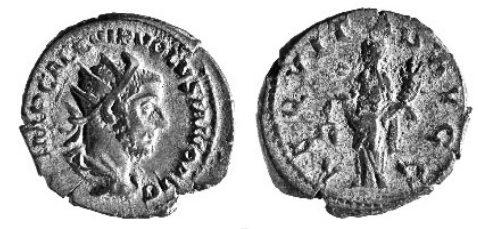

5
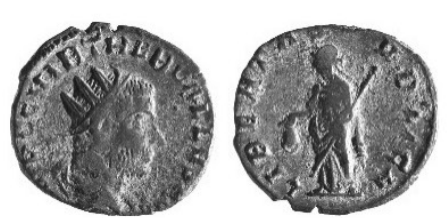

9

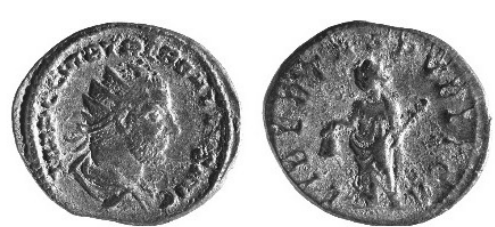

10

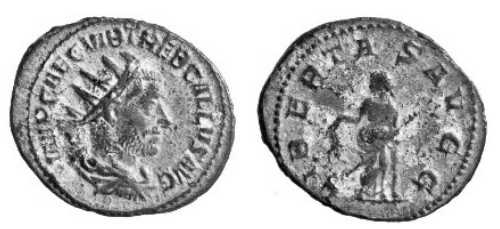

13

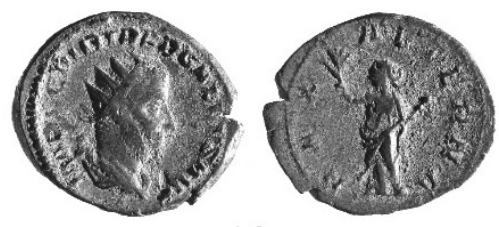

16

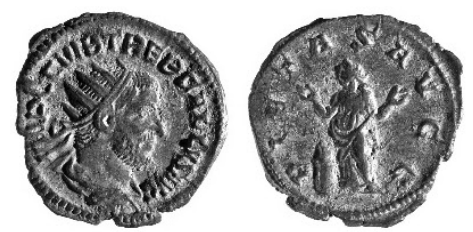

19

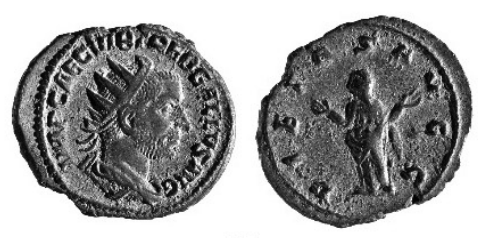

22

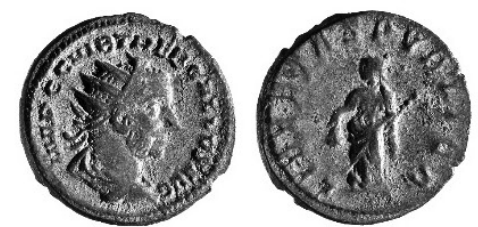

11

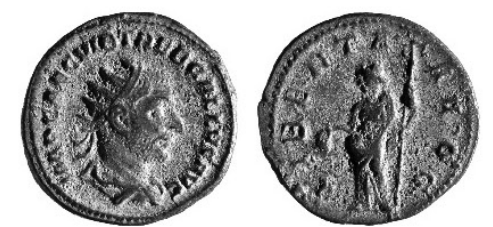

14

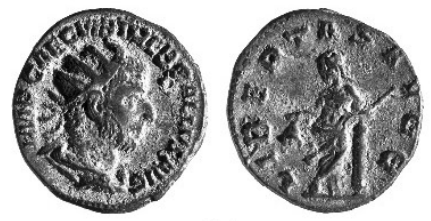

17

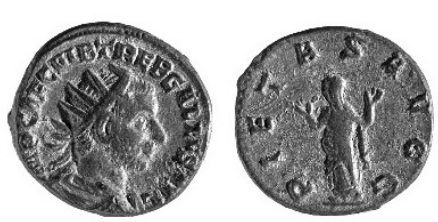

20
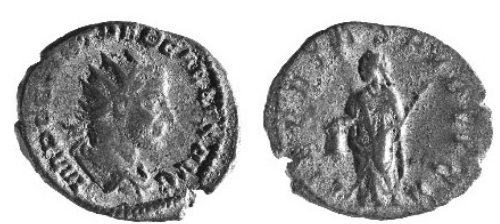

12

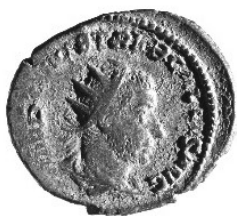

15
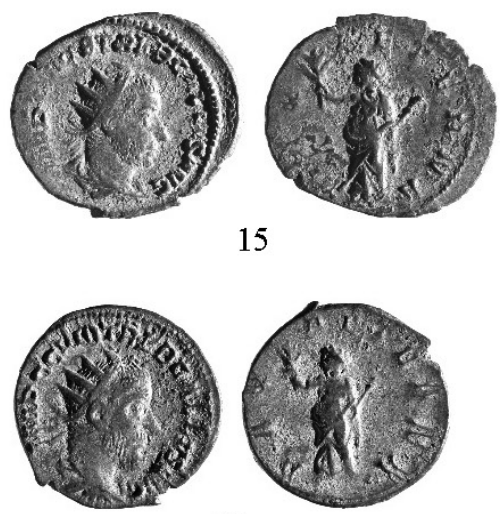

18
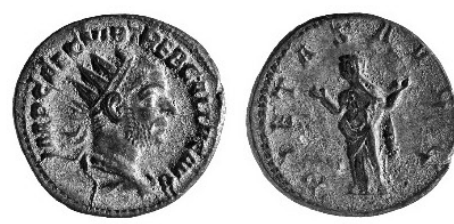

21
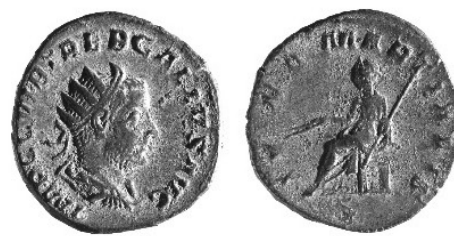

26 


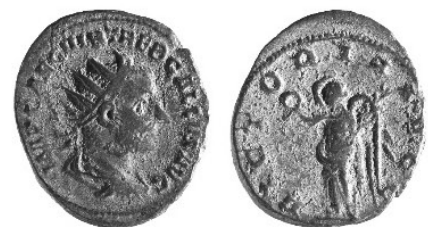

27

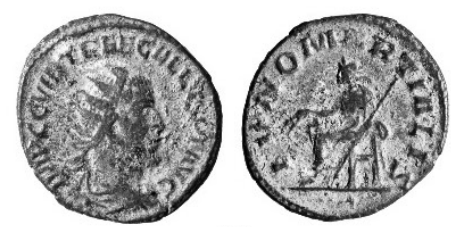

32

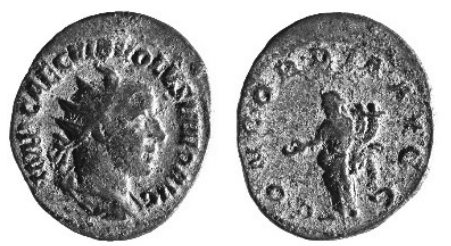

40

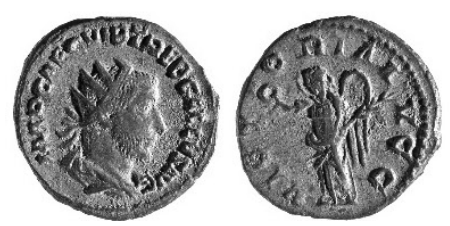

28

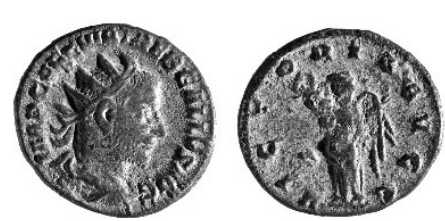

29

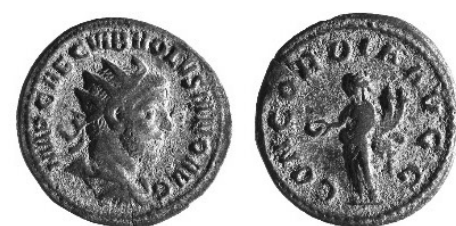

38

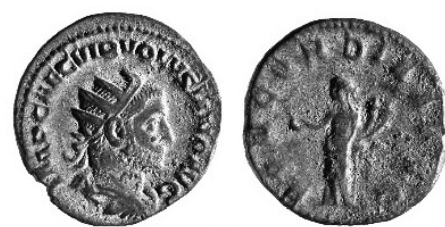

39

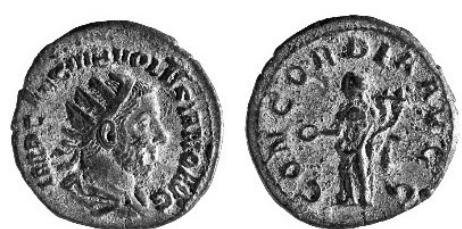

41
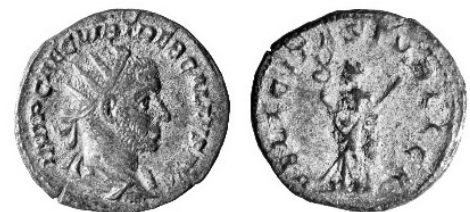

42

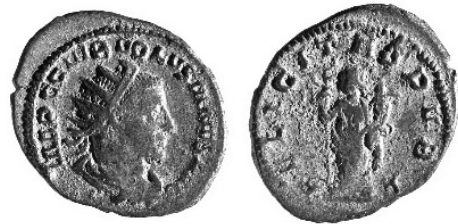

43

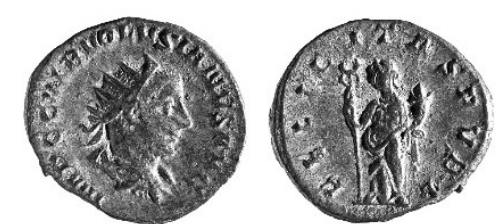

44

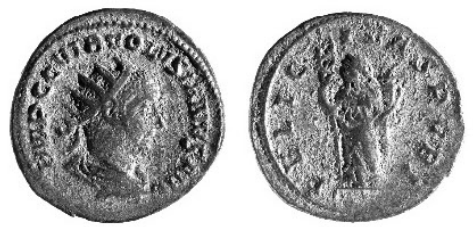

45

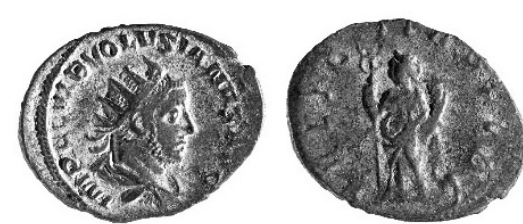

46

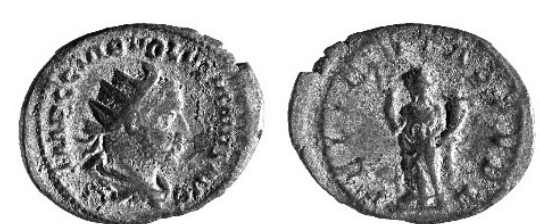

47

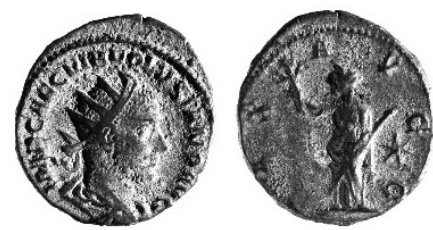

49

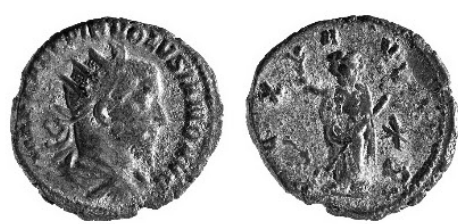

50

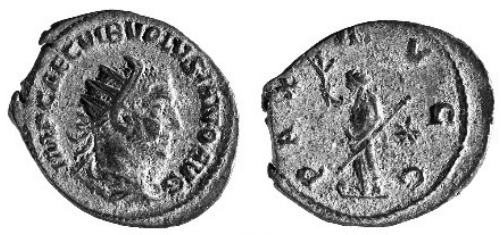

53

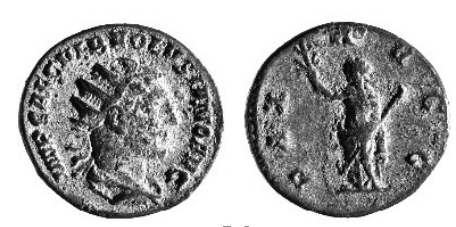

56

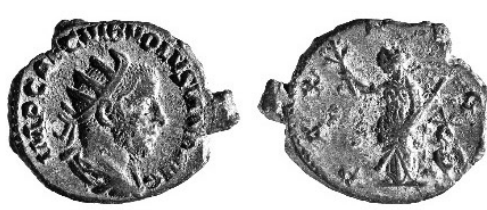

51

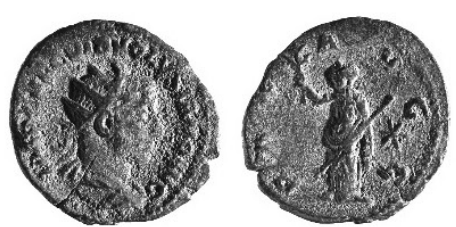

52

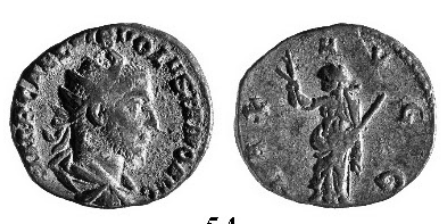

54

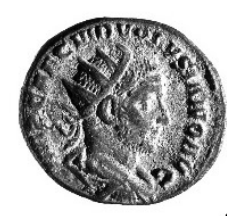

55

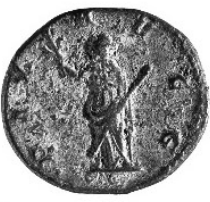

55

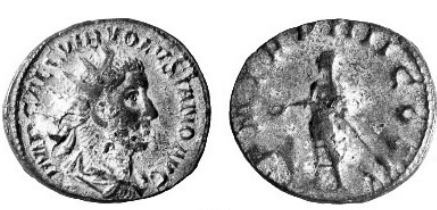

59 


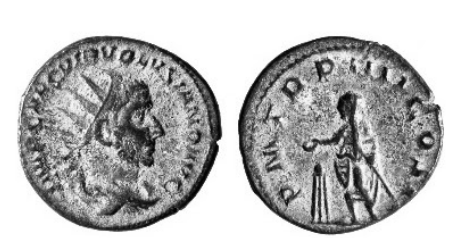

60

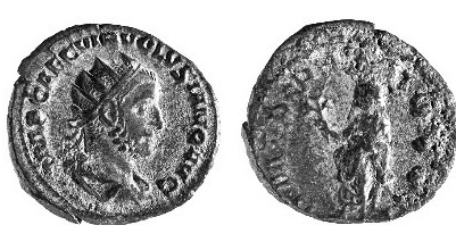

64

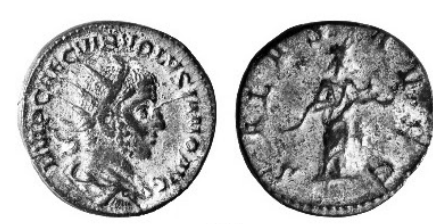

68

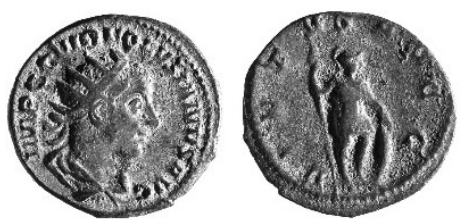

72

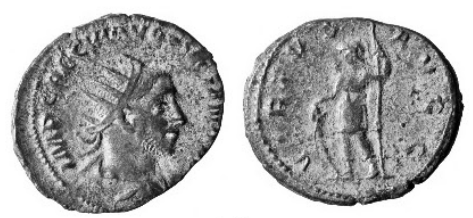

77

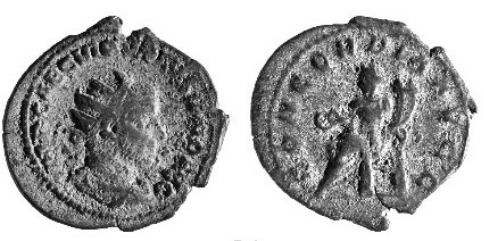

81

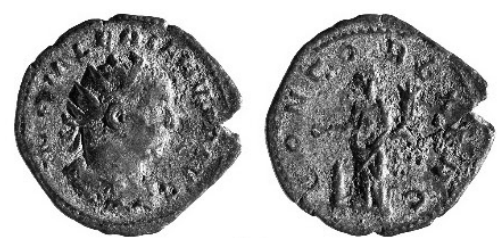

86

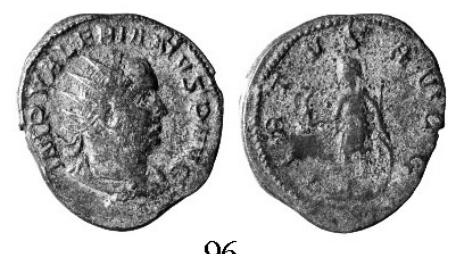

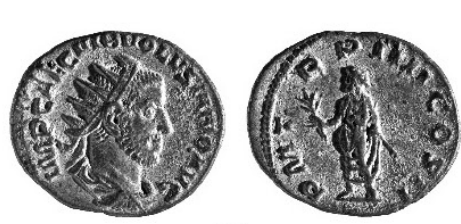

62

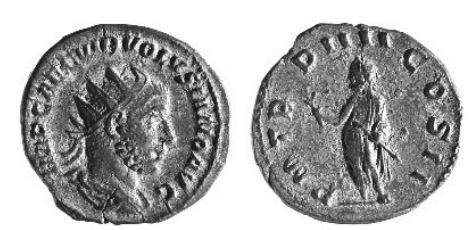

65

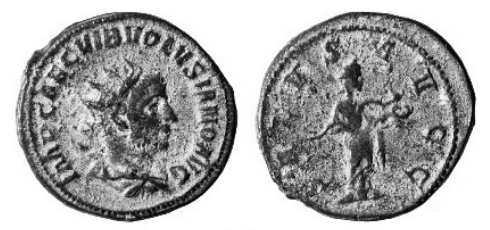

69

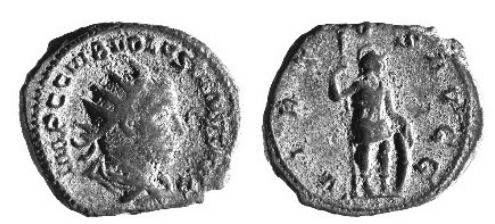

73

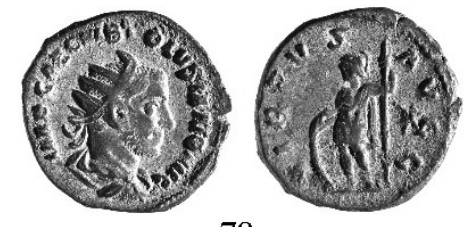

78

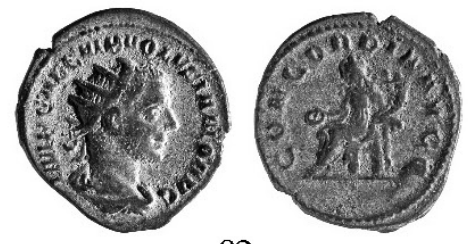

82

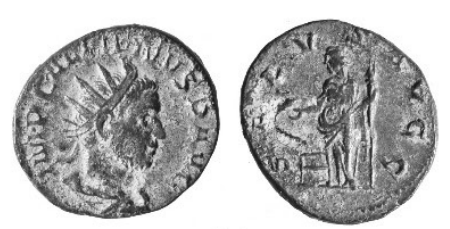

94

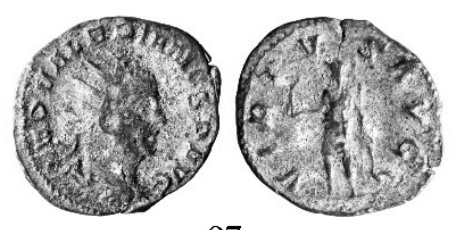

97

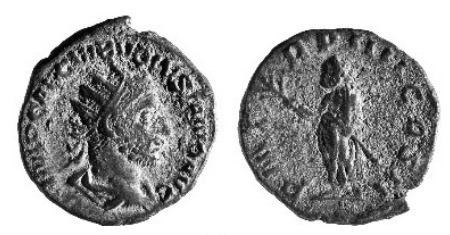

63
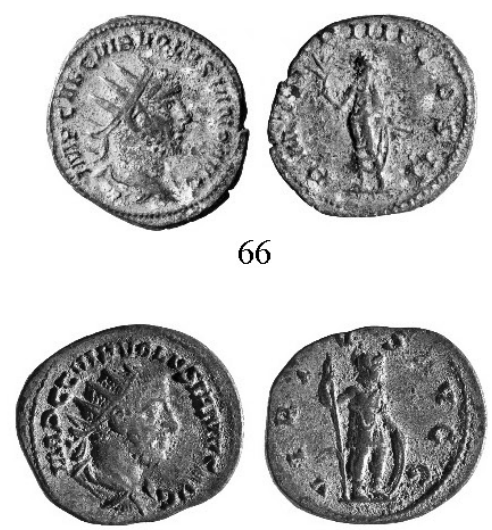

71

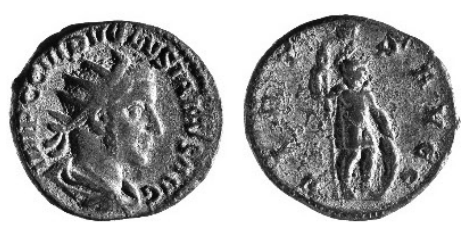

74

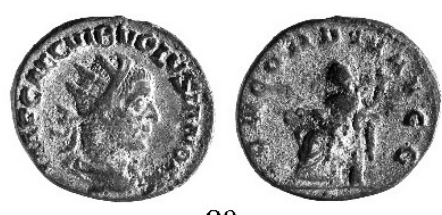

80

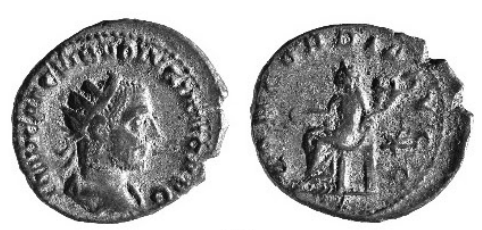

83

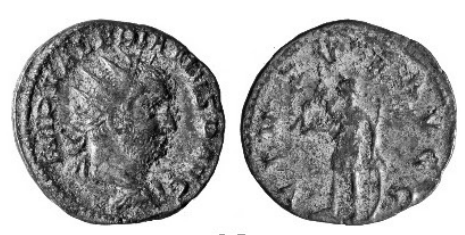

95

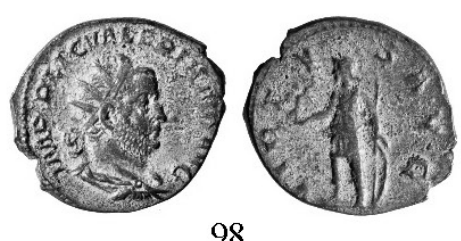



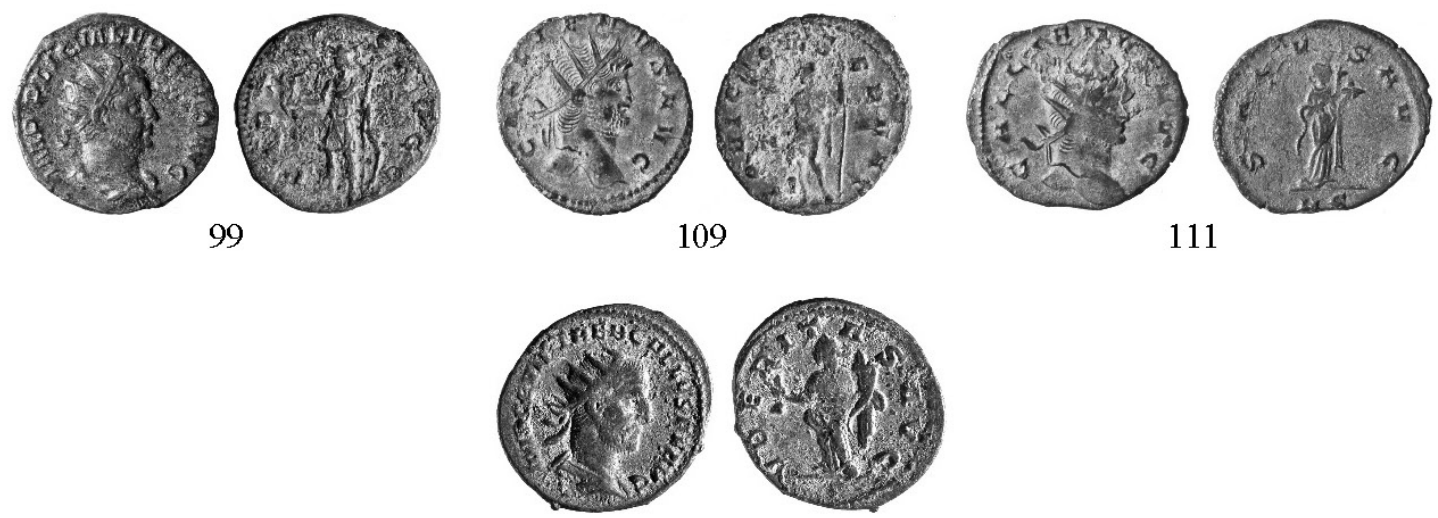

112 


\section{BIBLIOGRAFIA}

ASOLATI 1997

Asolati, M., Le collezioni Numismatiche: Legato Pietro Morosini, 1683 - Collezione San Giovanni di Verdara, 1783 - Legato Jacopo Nani, 1797 (quest'ultimo con Cristina Crisafulli). In: Favaretto, I./ Ravagnan, G.L. (a cura di), Lo statuario pubblico della Serenissima. Due secoli di collezionismo di antichità 1596-1797, catalogo della mostra (Cittadella (PD): Biblos), 246-257, 258-263, 264-266.

ASOLATI 2004

Asolati, M., Raccolte numismatiche. In: Favaretto, I./De Paoli, M./Dossi, M.C. (a cura di), Museo Archeologico Nazionale di Venezia (Milano: Electa), 155-161.

\section{ASOLATI 2009}

Asolati, M., Tremissi longobardi della raccolta del N.H. Domenico Pasqualigo nelle collezioni numismatiche del Museo Correr, Bollettino dei Musei Civici Veneziani s. III, 4, 70-73.

ASOLATI 2011

Asolati, M., Pigorini numismatico nei rapporti epistolari con Vincenzo Lazari e Carlo Kunz. In: Tra protostoria e storia. Studi in onore di Loredana Capuis, Antenor Quaderni 20 (Roma: Edizioni Quasar), 513535.

\section{ASOLATI/CRISAFULLI 1996}

Asolati, M., Crisafulli, C., Museo Archeologico di Venezia: proposta per una lettura della Collezione Numismatica, Archivio Veneto CXXVII, s. V, vol. CXLVI, 127-142.

\section{ASSANDRIA 1915}

Assandria, G., Lapide romana ed antichità romane rinvenute in Torino durante l'anno 1914, Notizie degli Scavi di Antichità, 61-64.

\section{AVERY 2012}

Avery, V., I bronzi Zen, Quaderni della Procuratoria VII, 72-83.

\section{BARELLO 2009}

Barello F., Il ripostiglio di Pombia riconsiderato. In: Barello, F./Spagnolo Garzoli, G. (a cura di), Mala tempora currunt. La crisi del III secolo attraverso il ripostiglio di Pombia, Atti della Giornata di Studi, Arona 24 novembre 2007 (Gravellona Toce: Ministero per i Beni e le Attività Culturali), 21-57.

\section{BASTIEN 1984}

Bastien, P., L'atelier de Milan en 268. In:
Gorini, G. (a cura di), La zecca di Milano.

Atti del Convegno Internazionale di Studio, Milano 9-14 maggio 1984 (Milano: Società Numismatica Italiana), 133-145.

\section{BERCHET 1899}

Berchet, F., Le Sale d'Armi del Consiglio dei Dieci nel Palazzo Ducale di Venezia (Venezia: Carlo Ferrari).

\section{BERNABEI 1988}

Bernabei, E., Ripostigli monetali in Italia. Schede Anagrafiche. Ceretto Lomellina (PV) 1927 e 1928 (Milano: ET).

\section{BESLY/BLAND1983}

Besly, E./Bland, R.F., The Cunetio Treasure. Roman Coinage of the Third Century AD (London: Trustees of the British Museum).

\section{CALZA 1914}

Calza, G., Ostia. Scavo presso l'edificio delle Piastrine. Scoperta di un monumento repubblicano e di un nuovo tipo di edificio privato. Tre nuove strade, Notizie degli Scavi di Antichità, 244-254.

\section{CARSON 1967-1968}

Carson, R.A.G., The Hamâ hoard and the Eastern mints of Valerian and Gallienus, Berytus XVII, 123-135.

\section{CESANO 1919}

Cesano, L., Sulla circolazione delle monete di bronzo nei primi tre secoli dell'impero romano. Ripostiglio del Testaccio, Atti e Memorie dell'Istituto Italiano di Numismatica III, 35-66.

\section{CHIARAVALLE 1986}

Chiaravalle, M., Ottobiano (PV), Ripostiglio di antoniniani di III secolo d.C., Bollettino di Numismatica 6-7, 296-299.

\section{CHIARAVALLE 1989}

Chiaravalle, M., Ripostigli monetali in Italia. Schede Anagrafiche. Ottobiano (PV) 1985 (Milano: ET).

\section{CHRISTOL 1975}

Christol, M., Les règnes de Valérien et de Gallien (253-268): travaux d'ensemble, questions chronologiques, in Temporini, H./ Haase, W. (a cura di), Aufstieg und Niedergang der Römischen Welt, II/2, Principat (BerlinNew York: de Gruyter), 803-827.

\section{COPE/KING/CLAY 1997}

Cope, L.H./King, C.E./Clay, T., Cope's Analyses. In: Cope, L.H./King, C.E./ Northover, J.P./Clay, T. (a cura di), Metal Analyses of Roman Coins minted under the Empire (London: British Museum), 1-66. 


\section{CORNAGGIA 1918}

Cornaggia, G., Gli antoniniani del III secolo nel ripostiglio di via S. Maria e Stampatori a Torino, Rivista Italiana di Numismatica XXXI, 234-269.

\section{CRISAFULLI 2006}

Crisafulli, C., Aggiornamenti sul ripostiglio di aurei del I secolo d.C. da Este. In: Bianchin Citton, E./Tirelli, M. (a cura di), ...ut... rosae...ponerentur. Scritti di archeologia in ricordo di Giovanna Luisa Ravagnan, Quaderni di Archeologia del Veneto, Serie speciale, 2 (Treviso: Edizioni Quasar - Canova), 137-142. CRISAFULLI 2008

Crisafulli, C., Economia monetaria in Italia alla vigilia del IV secolo d.C. Il ruolo dell'antoniniano e dei suoi omologhi gallici alla luce delle fonti numismatiche e storicoletterarie, Tesi di Dottorato, Università degli Studi di Padova (online al sito http:// paduaresearch.cab.unipd.it/view/people/ Crisafulli_Cristina.html).

\section{CRISAFULLI 2016}

Crisafulli, C., Radiati ufficiali e radiati irregolariinItaliaallafinedelIIIsecolo:alcune osservazioni. In: Chameroy, J./Guihard, P.-M. (a cura di), Produktion und Recyceln von Münzen in der Spätantike Produktion, 1. Internationales Numismatikertreffen (1516 mai 2014, Mainz) (Mainz: Verlag des Römisch-Germanischen Zentralmuseums), 71-82.

CRISAFULLI c.s.

Crisafulli, C., Il ripostiglio di Caraino rivisitato, Rivista Italiana di Numismatica, in corso di stampa.

DEL TORRE 1997

del Torre, G., "Dalli preti è nata la servitù di quella repubblica". Ecclesiastici e segreti di stato nella Venezia del '400. In: Gasparri, S./Levi, G./Moro, A. (a cura di), Venezia. Itinerari per la storia della città (Bologna: Il Mulino), 131-158

\section{DEMEGLIO 2003}

Demeglio, P., Ripostigli monetali in Piemonte tra età imperiale e altomedioevo: una schedatura. In: Lusuardi Siena, S. (a cura di), Fonti archeologiche e iconografiche per la storia e la cultura degli insediamenti nell'altomedioevo. Atti delle giornate di studio: Milano-Vercelli, 21-22 marzo 2002, Contributi di Archeologia 3 (Milano: V\&P Università), 173-195.

\section{DOLCINI 1999}

Dolcini, L., La collezione del cardinale Zen.

I frammenti fiamminghi. In: Dolcini, L./

Davanzo Poli, D./Vio, E. (a cura di), Arazzi della Basilica di San Marco (Milano: Rizzoli), 109-127.

\section{DRINKWATER 2005}

Drinkwater, J.F., Maximinus to Diocletian and the "crisis". In: Bowman, A.K./Garnsey, P./Cameron A. (a cura di), The Cambridge Ancient History, XII, The Crisis of Empire, A.D. 193-337 2nd Revised \& enlarged Edition (Cambridge: Cambridge University Press), 28-66.

\section{ELKS 1972}

Elks, K.J.J., Reattribution of the Milan Coins of Trajan Decius to the Rome Mint, The Numismatic Chronicle s. VII, XII, 111-115.

\section{ELMER 1941}

Elmer, G., Die Münzprägung der gallischen Kaiser in Köln, Trier und Mailand, Bonner Jahrbücher 146 (ristampa in forma autonoma Liege 1974).

\section{FAVARETTO 1990}

Favaretto, I., Arte antica e cultura antiquaria nelle collezioni venete al tempo della Serenissima, Studia Archaeologica 35 (Roma: "L'Erma" di Bretschneider).

\section{FAVARETTO 2012}

Favaretto, I., Il cardinale Giovanni Battista Zen, una enigmatica figura di prelato, Quaderni della Procuratoria VII, 60-71.

\section{FERRETTI 1986}

Ferretti, F., Piccola raccolta di antoniniani del terzo secolo provenienti dall'alto vercellese, Bollettino Storico Vercellese XV, 2, 23-68.

\section{FERRETTI 1994}

Ferretti, F., Un tesoretto di antoniniani da Gattinara, Bollettino Storico Vercellese XIII, 2, 118-173.

\section{FITZ 1978}

Fitz, J., Der Geldumlauf der römischen Provinzen im Donaugebiet Mitte des 3. Jahrhunderts (Budapest-Bonn: Akademiai Kiado).

FMRHR, XVIII

Miškec, A. 2002, Die Fundmünzen der Römischen Zeit in Kroatien, XVIII, Istrien (Mainz: Philipp von Zabern)

\section{GALLO 1950}

Gallo, B., Due placchette del Moderno e il busto di Francesco Novello da Carrara delle 
Sale delle Armi del Consiglio die X, Archivio Veneto s. V, XLVI-XLVII, 69-95

\section{GAMBIER 1988}

Gambier, M., Girolamo Ascanio Molin. In: Gambier, M. (a cura di), Una città e il suo museo. Un secolo e mezzo di collezioni civiche veneziane, catalogo della mostra, Bollettino dei Civici Musei Veneziani d'Arte e di Storia, n.s., XXX, 1986 (Venezia: Museo Correr Venezia), 91-94.

GARIBOLDI 2001

Gariboldi, A., Ripostigli monetali in Italia. Documentazione dei complessi. Il ripostiglio di Mortasa (Pavia) 1953 (Milano: Comune di Milano, Settore cultura musei e mostre, Civiche Raccolte Numismatiche).

GÖBL 1951

Göbl, R., Der Aufbau der römischen Münzprägung in der Kaiserzeit.V/1: Die Samtherrschrift von Valerianus und Gallienus (253-260), Numismatische Zeitschrift 22, 8-435.

\section{GORINI 1987}

Gorini, G., Aspetti monetali: emissione, circolazione e tesaurizzazione. In: Buchi, E. (a cura di), Il Veneto nell'età romana (Verona: Banca Popolare di Verona), 225-286.

\section{GORINI 1992,}

Gorini, G., I ripostigli di denari nella prima metà del terzo secolo nella Venetia et Histria. In Noeske, H.-C./Schubert, H. (a cura di), Die Münze. Bild-BotschaftBedeutung. Festschrift für Maria R.-Alföldi, (Frankfurt am Main - Bern - New York Paris: Peter Lang), 234-241.

\section{GORINI 1997}

Gorini, G., Lo statuario pubblico: il collezionismo numismatico. In: Favaretto, I./Ravagnan, G.L. (a cura di), Lo statuario pubblico della Serenissima. Due secoli di collezionismo di antichità 1596-1797, catalogo della mostra (Cittadella (PD): Biblos) 132-135.

\section{JESTAZ 1995}

Jestaz, B., Il caso di un cardinale veneziano: le committenze di Battista Zen a Roma e nel Veneto. In: Esch, A./Frommel, C.L. (a cura di), Arte, committenza ed economia a Roma e nelle corti del Rinascimento (1420-1530), Atti del Convegno Internazionale, Roma 2427 ottobre 1990 (Torino: Piccola Biblioteca Einaudi), 331-352.

KING/NORTHOVER 1997
King, C.E./Northover, J.P., Ashmolean, British Museum and Neftenbach Hoard analyses. In: Cope, L.H./King, C.E./ Northover, J.P./Clay, T. (a cura di), Metal Analyses of Roman Coins minted under the Empire (London: British Museum), 69-119.

\section{LE GENTILHOMME 1946}

Le Gentilhomme, P., La trouvaille de Nanterre, Revue Numismatique s. V, IX, 15114.

\section{LE GENTILHOMME 1962}

Le Gentilhomme, P., Variations du titre de l'antoninianus au IIIe siècle, Revue Numismatique s. VI, IV, 141-166.

\section{MARTANI 1992}

Martani, M., Lodivecchio - Di un ripostiglio di monete imperiali rinvenuto nel territorio del Comune, Notizie degli Scavi di Antichità, 191.

MARTINI 1995

Martini, R., Ripostigli monetali in Italia. Documentazione dei complessi. Il ripostiglio di Ceretto Lomellina (Pavia), 1927-28 (Milano: Mascher).

MIR, 36

Göbl, R. 2000, Die Münzprägung der Kaiser Valerianus I/Gallienus/Saloninus (253/258), Regalianus (260) und Macrianus/Quietus (260/262), Moneta Imperi Romani 36, 43, 44 (Wien: Verlag der Österreichische Akademie der Wissenschaften).

MORATI 1871

Morati, F., Informazione sopra alcuni ripostigli di monete, Periodico di Numismatica e sfragistica per la Storia d'Italia III, 58-69.

MORETTI 1922

Moretti, G., Falerone - Ripostiglio monetale rinvenuto nell'area dell'antico Falerio, Notizie degli Scavi di Antichità, 59-76.

PATIN 1683

Patin, C., Thesaurus Numismatum Antiquorum \& Recentiorum, ex Auro, Argento, \& Aere, Ab Illustriss. \& Excellentiss. D. D. Petro Mauroceno, Senatore Veneto, Serenissimae Reipublicae Legatus (Venetiis: ex typographia Io. Francisci Valuasensis).

PENSA 1984

Pensa, M., Il tesoretto di S. Michele in Lodivecchio, Archivio Storico Lodigiano (pubbl. nel 1985), 29-139.

\section{PERANTONI SATTA 1954}

Perantoni Satta, G., Rinvenimenti in Sardegna di monete dell'Impero romano 
e dell'Impero romano d'Occidente. I Ripostigli, Annali dell'Istituto Italiano di Numismatica 1, 65-111.

\section{RAVAGNAN 1997}

Ravagnan, G.L., Le ultime vicende dello Statuario Pubblico dalla caduta della Repubblica di Venezia alla nascita del Museo Archeologico Nazionale. In: Favaretto, I./Ravagnan, G.L. (a cura di), Lo statuario pubblico della Serenissima. Due secoli di collezionismo di antichità 1596-1797, catalogo della mostra (Cittadella (PD): Biblos), 66-73.

\section{REECE 1971}

Reece, R., Roman Coinage in Northern Italy, Numismatic Chronicle s. VII, XI, 167-179.

RIC

The Roman Imperial Coinage, voll. I-X (London: Spink \& Son) 1923-2007.

RMRVE, III/3

Pavoni, M.G. 2005, Ritrovamenti Monetali di età Romana nel Veneto, III/3, Provincia di Verona: Peschiera del Garda (Padova: Esedra Editrice).

\section{SCAFILE 1976}

Scafile, F., Un tesoretto monetale rinvenuto a Pombia, Ad Quintum 4 (giugno), 56-82.

SORANZO 1962

Soranzo, G., Giovan Battista Zeno, nipote di Paolo II, cardinale di S. Maria in Portico (1468-1501), Rivista di storia della Chiesa in Italia 16, 249-274.

\section{TARAMELLI 1931}

Taramelli, A., Porto-Torres. Tomba di età imperiale con armilla d'oro e monete d'argento di Caracalla e Gallieno, rinvenuta nell'abitato, Notizie degli Scavi di Antichità, 108-111.

\section{TRAVAINI 1985}

Travaini, L., Sito numismatico C. Il ripostiglio del Testaccio (via Bodoni 1911), Bollettino di Numismatica 5 (lugliodicembre), 153-155.

\section{TYLER 1975}

Tyler, P., The Persian Wars of the $3^{\text {rd }}$ Century and Roman imperial monetary policy, A.D. 253-268, "Historia", Einzelschriften 23 (Wiesbaden: F. Steiner).

\section{VISMARA 1994}

Vismara, N., Le collezioni numismatiche dei Civici Musei di Pavia, Bollettino della Società Pavese di Storia Patria, 341-356.

VISMARA 2002

Vismara, N., Ripostigli monetali in Italia. Documentazione dei complessi. Il ripostiglio di Pavia 1868 (Milano: Comune di Milano, Settore musei e mostre, Civiche Raccolte Numismatiche).

\section{ZIPPEL 1904}

Zippel, G. (a cura di), Le vite di Paolo II di Gaspare da Verona e Michele Canensi, Rerum Italicarum Scriptores, Raccolta degli storici italiani da cinquecento al millecinquecento, ordinata da L.A. Muratori, nuova edizione riveduta ampliata e corretta, con la direzione di Giosuè Carducci e Vittorio Fiorini, III, XVI (Città di Castello: coi tipi dell'editore S. Lapi). 
